University of Louisville

ThinkIR: The University of Louisville's Institutional Repository

$5-2020$

\title{
Modulation of host innate immune cells by Yersinia pestis to create a permissive environment for replication.
}

Amanda Rose Pulsifer

University of Louisville

Follow this and additional works at: https://ir.library.louisville.edu/etd

Part of the Pathogenic Microbiology Commons

\section{Recommended Citation}

Pulsifer, Amanda Rose, "Modulation of host innate immune cells by Yersinia pestis to create a permissive environment for replication." (2020). Electronic Theses and Dissertations. Paper 3384.

https://doi.org/10.18297/etd/3384

This Doctoral Dissertation is brought to you for free and open access by ThinkIR: The University of Louisville's Institutional Repository. It has been accepted for inclusion in Electronic Theses and Dissertations by an authorized administrator of ThinkIR: The University of Louisville's Institutional Repository. This title appears here courtesy of the author, who has retained all other copyrights. For more information, please contact thinkir@louisville.edu. 


\title{
MODULATION OF HOST INNATE IMMUNE CELLS BY YERSINIA PESTIS TO CREATE A PERMISSIVE ENVIRONMENT FOR REPLICATION
}

\author{
By \\ Amanda Rose Pulsifer \\ B.S., Bloomsburg University of Pennsylvania, 2012 \\ M.S., University of Louisville, 2015 \\ A Dissertation \\ Submitted to the Faculty of the \\ School of Medicine of the University of Louisville \\ in Partial fulfillment of the Requirements \\ for the Degree of \\ Doctor of Philosophy \\ in Microbiology and Immunology \\ Department of Microbiology and Immunology \\ University of Louisville School of Medicine \\ Louisville, KY
}

May 2020 
Copyright 2020 by Amanda Rose Pulsifer

All rights reserved 



\title{
MODULATION OF HOST INNATE IMMUNE RESPONSES BY YERSINIA PESTIS TO CREATE A PERMISSIVE ENVIRONMENT FOR REPLICATION
}

\author{
By \\ Amanda Rose Pulsifer \\ B.S., Bloomsburg University of Pennsylvania, 2012 \\ M.S., University of Louisville, 2015 \\ A Dissertation Approved on
}

March 13, 2020

by the following Dissertation Committee:

\begin{tabular}{c}
\hline $\begin{array}{c}\text { Dissertation Director } \\
\text { Matthew B. Lawrenz }\end{array}$ \\
\hline Silvia M. Uriarte \\
\hline Yousef Abu-Kwaik \\
\hline Brian P. Ceresa \\
\hline Jonathan M. Warawa
\end{tabular}




\section{DEDICATION}

The following discourse is the result of numerous frustrating failures, exciting results, dashed hopes and dreams, newly formed hopes and dreams, proclamations of experimental completion (only to return to the bench for "one more experiment"), and many nights of sleep lost. From start to finish, this has been an experience filled with many emotions. This work is dedicated to Tammy (Mom), Tiva, and Praveen, who have endured this journey with me, picking me up from the lows and celebrating with me during the highs. Earning a Ph.D. was not a goal of mine when I was young, but during this process my love for pursuing science has only strengthen, and I have learned I am capable of achieving more than I ever dreamed. 


\section{ACKNOWLEDGMENTS}

To begin I would like to thank Matthew Lawrenz for welcoming me into his lab, and for giving me the freedom to pursue the questions I found interesting. My development as a scientist and presentation skills would not have occurred without his patient guidance. Developing as a scientist requires branching out, learning new fields, and continually growing. I will be forever grateful to Matt for ensuring a solid foundation upon which to build, has been developed. The Lawrenz lab has seen a number of faces come and go during my time in the lab. Tiva VanCleave and Michael Connor were who I turned to whenever I needed advice, I couldn't have asked for better friends/lab family. Sarah, Amanda and Jen have replaced Tiva and Michael and will carry the lab forward to new discoveries.

I would like to thank my committee members for their questions and advice during the course of my studies. They have continually pushed me to think, and become a better scientist. Silvia Uriarte stepped in as my co-advisor and has been encouraging every step of the way, always looking out for my best interest and helping me to achieve more than I thought I was capable of.

My family has also been there for me every step of the way and I am so grateful for all of them. While I was far from my family during graduate school, the friends I made were like my adopted family. I have never laughed so hard and enjoyed life as much as since l've met each of you! There are too many to list, but you know who you are my fellow Gremlins and Makenzie! 


\section{ABSTRACT \\ MODULATION OF HOST INNATE IMMUNE CELLS BY YERSINIA PESTIS TO CREATE A PERMISSIVE ENVIRONMENT FOR REPLICATION}

Amanda R. Pulsifer

March 13, 2020

Yersinia pestis has gained widespread infamy due to the historic outbreak during the middle ages, referred to as The Black Death. Infection with Y. pestis typically begins with deposition of Y. pestis into the dermis (bubonic plague) or respiratory tract (pneumonic plague). Tissue resident macrophages are the first innate immune cell encountered by $Y$. pestis. Macrophages are likely a way for Y. pestis to avoid neutrophils early in infection when the neutrophil neutralizing Type Three Secretion System is not expressed. This work focuses on which Rab host proteins are manipulated by $Y$. pestis, and how neutrophils are forced to remain silent when all alarms and the arsenal they possess should be triggered. Through an RNAi screen 13 of 45 screened Rab proteins were found to be important for intracellular Y. pestis survival. The Rab proteins were prioritized based on the impact gene knockdown had on Y. pestis intracellular survival. Overexpressed Rab2b and Rab20 co-localized to the YCV, while overexpressed Rab13 did not. Indicating Rab13 may regulate $Y$. pestis intracellular survival in a contact independent manner. Survival within macrophages likely provides $Y$. pestis time to express the type three secretion system. Using deletion and addition mutants, I found that $Y$. pestis uses the type three secretion system effectors, YopE, YopH, YopJ, and YpkA to inhibit neutrophil degranulation, in addition to inhibiting LTB4 production in human neutrophils also by YopT. Unlike human neutrophils, LTB4 is not produced in response to $Y$. pestis in mouse neutrophils or macrophages and the zinc binding protein, calprotectin is released in vivo, but not by human neutrophils. Together, the Rab data and neutrophil exocytic responses contribute to our understanding of how $Y$. pestis manipulates host phagocytic cells to create a permissive environment in which to survive and replicate. 


\section{TABLE OF CONTENTS}

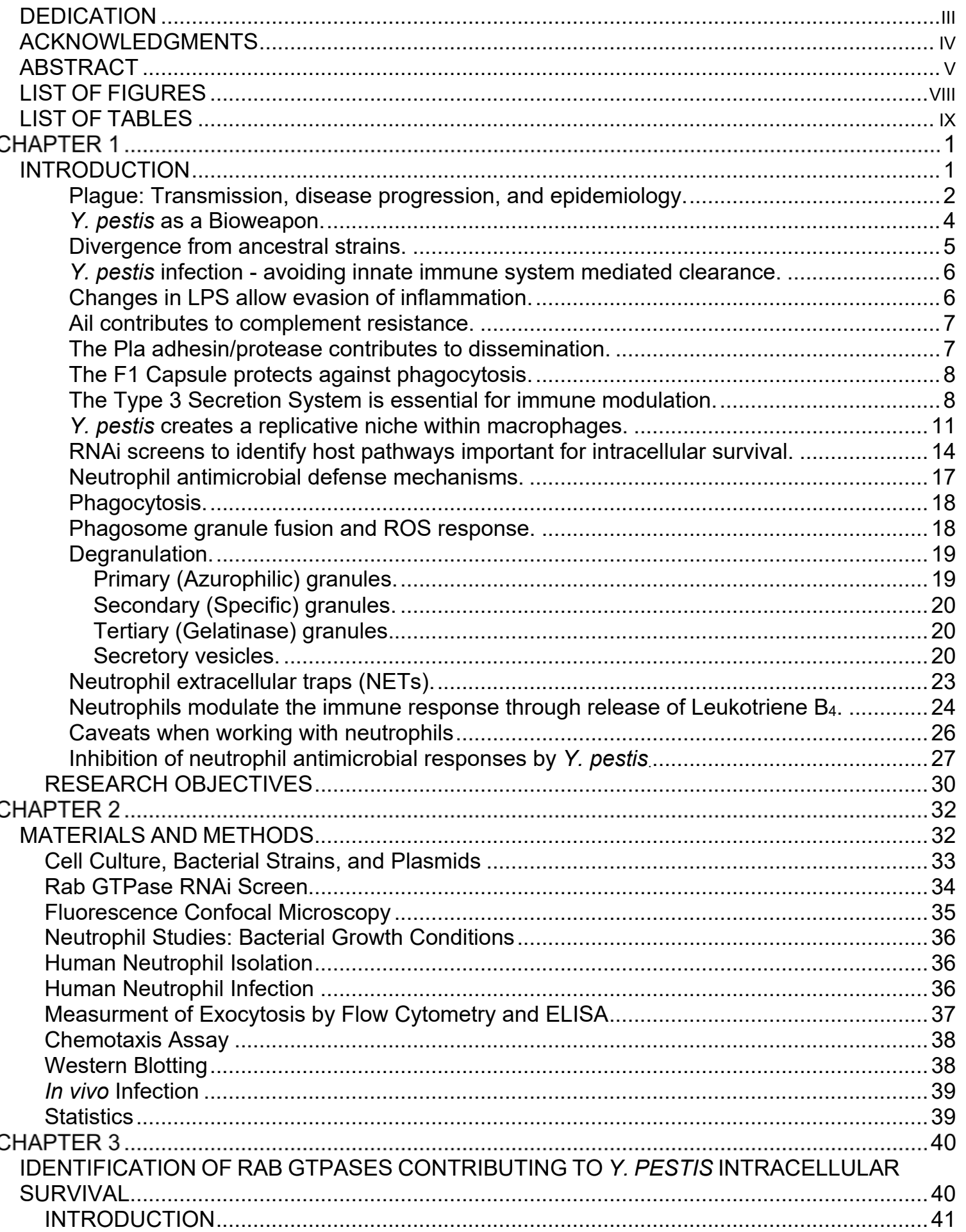


RESULTS

RNAi screen identifies Rab GTPases required for Y. pestis survival within macrophages.

Y. pestis survival within macrophages. 43

Rab GTPases are differentially localized to the YCV. .................................................... 44

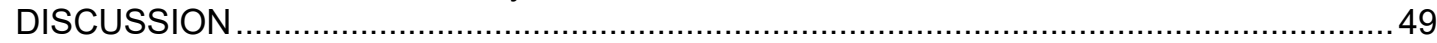

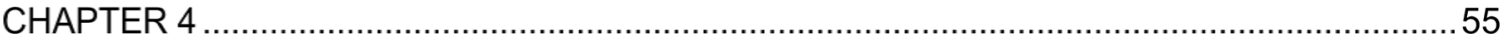

REDUNDANT AND COOPERATIVE ROLES FOR YERSINIA PESTIS YOP EFFECTORS IN THE INHIBITION OF HUMAN NEUTROPHIL EXOCYTIC RESPONSES REVEALED BY GAIN-

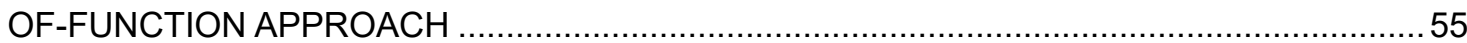

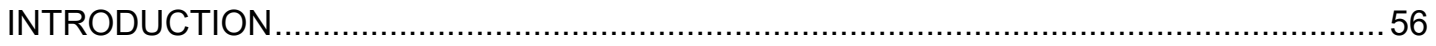

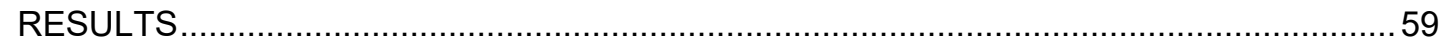

Y. pestis inhibits neutrophil degranulation in a T3SS-dependent manner........................59 Cooperative inhibition of neutrophil degranulation by Yop effectors revealed through gain-

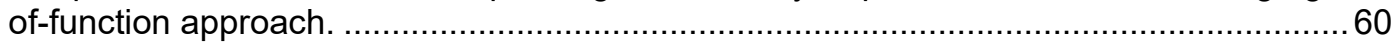

YopE, YopH, YopJ, and YpkA act cooperatively to inhibit degranulation of specific and

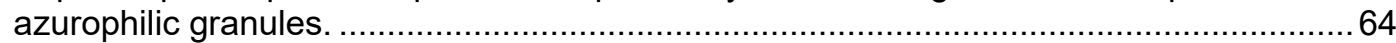

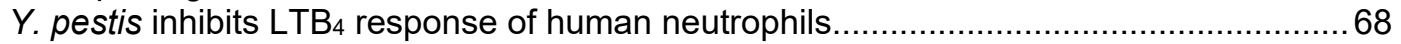

Disruption of the host cytoskeleton inhibits $\mathrm{LTB}_{4}$ release in response to $Y$. pestis infection.

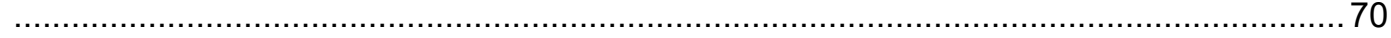

Disruption of MAPK signaling inhibits LTB 4 synthesis in response to $Y$. pestis infection. .71

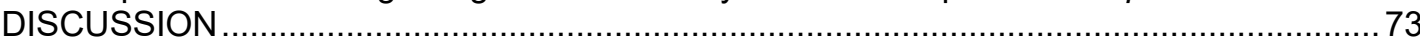

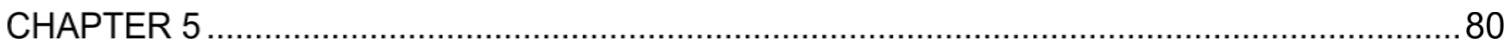

ADDITIONAL OBSERVATIONS, DISCUSSION, AND FUTURE DIRECTIONS ...................... 80

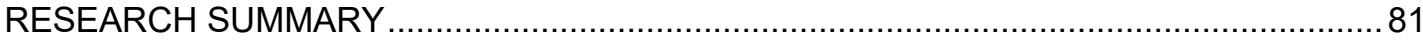

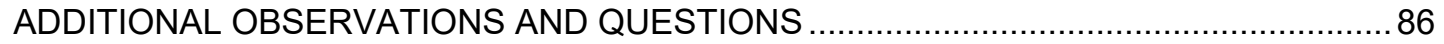

5.1 How does $Y$. pestis survive and replicate within macrophages? .............................. 86

5.1.1 Hypothesis 1: Y. pestis encodes effector proteins to modulate Rab protein

trafficking...................................................................................................... 86

5.1.2 Hypothesis 2: Y. pestis binds to a specific receptor to avoid phagosome

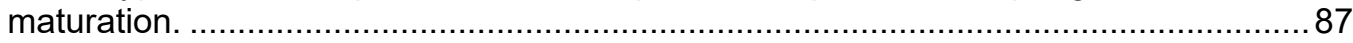

5.2 How are neutrophil antimicrobial responses altered during Y. pestis infection? .......... 89

5.2.1 Which host proteins do Yop effectors interact with in human neutrophils to inhibit

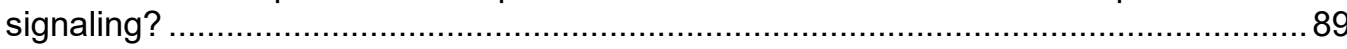

5.2.2 How does Y. pestis inhibit the synthesis of LTB 4 ? ............................................ 90

5.2.3 What impact does YopJ inhibition of p38 phosphorylation have on neutrophil

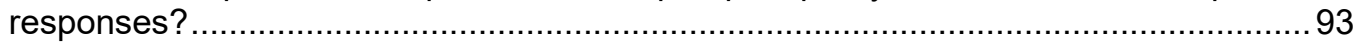

5.2.4 Does Y. pestis inhibit release of NETs by neutrophils? .................................... 95

5.2.5 Does the exosome profile released by neutrophils change upon infection with $Y$.

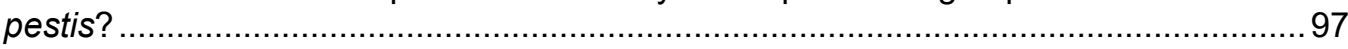

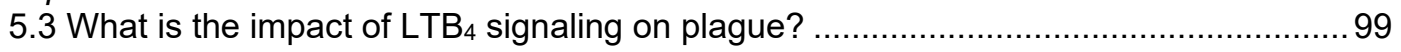

5.3.1 Does exogenous addition of $\mathrm{LTB}_{4}$ enhance the degradative capacity of innate

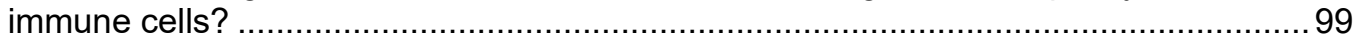

5.3.2 Could administration of $\mathrm{LTB}_{4}$ enhance host resistance to pneumonic plague? ...99

5.4 Do murine neutrophils release $\mathrm{LTB}_{4}$ in response to $Y$. pestis? ................................ 101

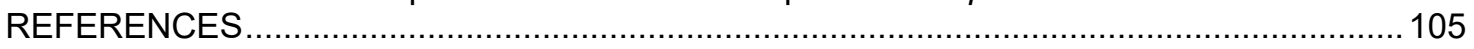

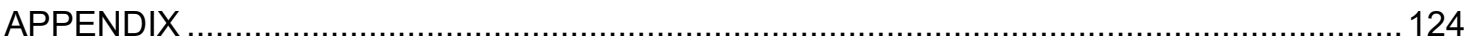

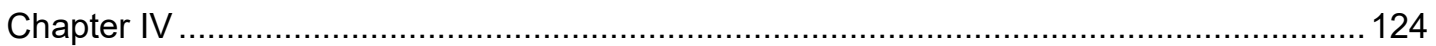

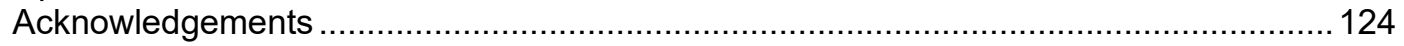

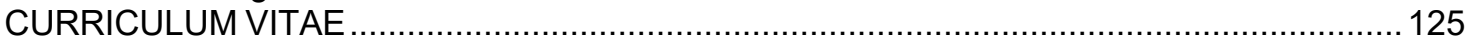




\section{LIST OF FIGURES}

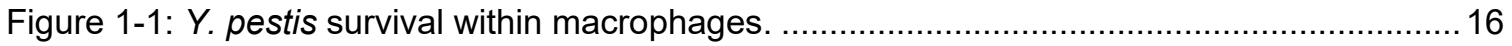

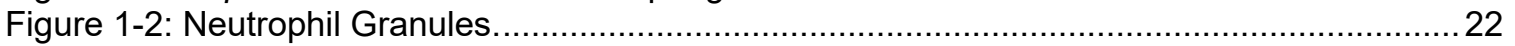

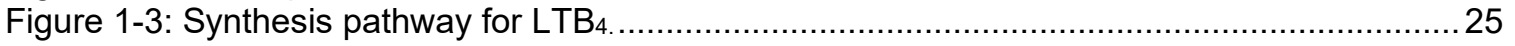

Figure-1-4: Y. pestis Yop Effector Protein Impact on Neutrophil Antimicrobial Responses..........29

Figure-3-1: Rab proteins identified as essential for $Y$. pestis intracellular survival. ......................46

Figure-3-2: Rab proteins identified as essential for $Y$. pestis intracellular survival from de-

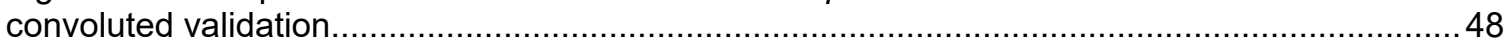

Figure 3-3: Rab2b, Rab13, and Rab20 co-localization in RAW264.7 macrophages. ...................51

Figure-4-1: Y. pestis inhibits degranulation in a T3SS-dependent manner. ................................61

Figure-4-2: Degranulation of specific and azurophilic granules peaks by $30 \mathrm{~min}$ post-infection...62 Figure-4-3: Deletion of individual Yop effector proteins does not alter neutrophil degranulation

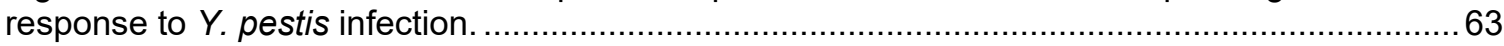

Figure-4-4: Individual Yop effector proteins are unable to completely inhibit degranulation.........65

Figure 4-5: At least two Yop effector proteins are required to fully inhibit specific granule release.

Figure 4-6: At least two Yop effector proteins are required to fully inhibit azurophilic granule

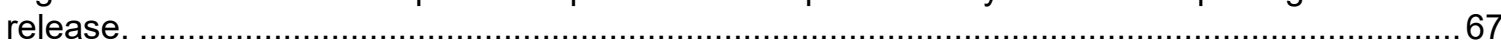

Figure 4-7: Y. pestis inhibits human neutrophil LTB4 response............................................69

Figure 4-8: Inhibition of cytoskeletal rearrangement or MAPK signaling inhibits $\mathrm{LTB}_{4}$ release.....72

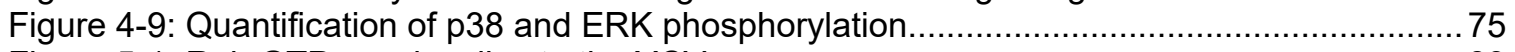

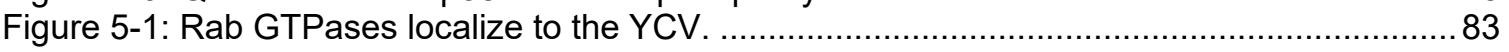

Figure 5-2: Inhibition of neutrophil granule release by Y. pestis................................................... 84

Figure 5-3: Inhibition of LTB4 production by T3SS effector proteins ........................................ 85

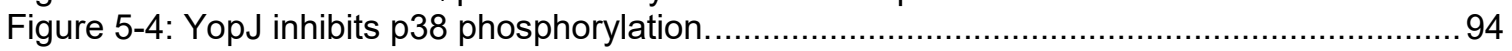

Figure 5-5: Y. pestis inhibits release of calprotectin from human neutrophils. ............................... 96

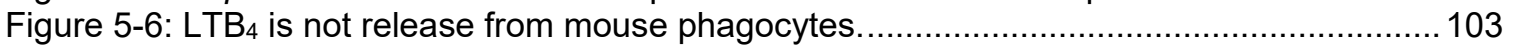




\section{LIST OF TABLES}

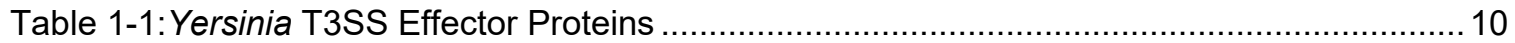

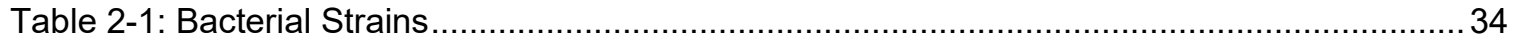


CHAPTER 1

INTRODUCTION 
Plague: Transmission, disease progression, and epidemiology.

Yersinia pestis is an adept pathogen with a well-documented history of causing disease in human populations, manifesting as the disease known as plague. Infection with $Y$. pestis results in a variety of signs and symptoms contingent upon the route of inoculation. Y. pestis is a vector borne pathogen, passing from rodents to accidental human hosts through the bite of infected fleas [1]. The ability of $Y$. pestis to be transmitted by an insect vector was acquired after divergence from the enteric pathogens $Y$. enterocolitica and Y. pseudotuberculosis [2]. Flu-like symptoms are the first warning signs of plague, with body aches, fever, chills, and other symptoms [1].

When Y. pestis is transmitted through flea bite into the skin, the form of plague that arises is referred to as bubonic plague. Bubonic plague is approximately $30-60 \%$ lethal in infected individuals without antibiotic treatment, with an infectious dose ranging from as little as one to one hundred bacteria [1]. As Y. pestis disseminates from the site of inoculation and colonizes the draining lymph node, the hallmark buboes begin to form, from which the name bubonic plague is derived, as $Y$. pestis proliferates in the lymph node to high numbers [3, 4].

Septicemic plague occurs when Y. pestis enters the blood upon dissemination, or direct inoculation of $Y$. pestis into the blood during flea feeding. Upon entering the blood, a required part of the transmission process, Y. pestis spreads throughout the host quickly colonizing major areas of blood filtration such as the spleen, liver and lungs [5,6]. Septicemic plague is highly lethal, killing $\sim 100 \%$ of untreated patients within 72 hours [1]. As the bacteria overtake the host, tissues in the extremities begin to undergo necrosis, causing a visible blackening of the tissues.

There are two classifications of pneumonic plague, which are differentiated by the way in which Y. pestis enters the lungs. Primary pneumonic plague arises when the bacteria enter the lungs from an exogenous source, such as inhaling aerosolized droplets containing $Y$. pestis. Secondary pneumonic plague arises from $Y$. pestis entering the lungs via the blood, subsequent to either disseminated bubonic or primary septicemic infection. Similar to septicemic plague, pneumonic plague is $\sim 100 \%$ lethal within 72 hours without antibiotic administration [1]. Pneumonic 
plague can also result in human to human spread, through aerosols generated by coughs and sneezes.

Three Y. pestis pandemics have been classified. The first pandemic is referred to as the Plague of Justinian, and began in the $6^{\text {th }}$ century and lasted approximately 200 years [7]. The $14^{\text {th }}$ century (Middle Ages) saw the rise of one of the most noted plague outbreak events, referred to as the Black Death. Plague contributed to extensive loss of human life as the pandemic swept through western Asia and Europe and flare up outbreaks continued well into the 1600s [8]. Studies have isolated $Y$. pestis DNA from the teeth of individuals buried in mass graves who died during this time period, confirming Y. pestis caused was the cause of the human casualties [9]. Many estimate the European population was reduced by $1 / 4^{\text {th }}$ to $1 / 3^{\text {rd }}$ during the Black Death [10]. The third pandemic began around 1896 and is still ongoing today. Cases of plague sporadically occur around the globe as spillover events due to $Y$. pestis now being endemic in rodent populations.

How $Y$. pestis has spread across the globe has not been fully elucidated and is the topic of many studies. China is believed to be the source from whence all three pandemics originated [11]. For the Plague of Justinian and the Black Death it is thought that the Silk Road was the route from which Y. pestis spread into Europe [12]. The Silk Road was the main trading route for the time and was a thoroughfare for transporting goods through and out of China. The Silk Road connected China to eastern Asia, and eventually Europe, via the Mediterranean. Incidences of plague outbreaks were reported to have occurred along the Silk Road route, supporting the idea that the Silk Road was likely the pathway for introduction of plague into Europe.

A direct land route was not possible for movement of $Y$. pestis into the Americas. However, ships laden with goods from China arrived in the ports of California, and thus introduced plague into the Americas [13]. From the Californian ports, Y. pestis radiated out into the native rodent populations of western North America, finding a new reservoir in prairie dogs, squirrels, and other American rodents [14]. Y. pestis has become a blight on two American animal populations, decimating prairie dog colonies and spilling over into the black footed ferret population, whom pray upon prairie dogs [15]. Y. pestis not only kills susceptible black footed ferrets, but those fortunate 
enough to avoid infection, face ultimate demise when prairie dog populations, the sole food source of black footed ferrets, dwindle. Conservation efforts to re-establish the endangered black footed ferret populations have been hindered by $Y$. pestis outbreaks in prairie dog colonies [16]. A number of spill over events from rodents into human populations occur each year in the American west, acting as a detriment not only to rodent populations and black footed ferret populations, but humans as well [17].

While North America records several human plague cases yearly, Madagascar usually records the most human plague cases each year [18]. Wild rodents on the island harbor Y. pestis and spill over events occur yearly [19]. The worst outbreak of plague in recent years was from the Fall of 2017, when more than 2,000 cases of plague were reported $[20,21]$. The disease was first transmitted to humans through fleas, but human to human transmission via pneumonic plague was the main driver of this outbreak [20]. Early cases were first recorded in rural areas, but as humans traveled, cases began to appear in the capital city of Antananarivo [20, 21]. With pneumonic plague cases in the crowded capital city, the number of cases increased until mechanisms to prevent the spread were implemented. The 2017 Madagascar outbreak is fresh in our memory, but outbreaks in India, China and South America also occur annually [22, 23]. Climate change has the potential to change reservoir host habitats that could increase potential human spill over events. Lack of an approved vaccine for human use, continues to leave human populations around the globe vulnerable to outbreaks of plague.

\section{Y. pestis as a Bioweapon.}

Y. pestis has unfortunately also been used as a biological weapon [24]. During the War of Kaffa, plague infected corpses were tossed over the walls of the besieged city by the invading Tartar army in the hopes that the disease would be transmitted to inhabitants [25]. A more recent use was during World War II, when Japan performed research on prisoners of war using Y. pestis to study how quickly people die and how it could be used to against foreign nations. Additionally, Unit 731 of Japan made possible the utilization of $Y$. pestis as a "biological bomb" by dropping rice mixed with Y. pestis infected fleas over China [25]. Due to study and use of Y. pestis as a biological 
weapon during WWII, it has been estimated that several thousand individuals were infected, with recurrent outbreaks of plague in subsequent years [26]. Japan was not the only modern country to weaponize Y. pestis. During the cold war, both Russia and the United States had active programs working to create aerosolized Y. pestis to target the opposition. In addition, Russia was also pursuing ways in which to increase the lethality of $Y$. pestis infection by attempting to combine $Y$. pestis with toxins or viruses. The plan was for the toxins or viruses to release upon treatment with antibiotics due to lysis of $Y$. pestis [25].

While neither country has active biological weapons programs now, concerns that $Y$. pestis could be used again as a bioweapon still remain. Counter-terrorism has been a top priority for the United States government, but the efficacy of such measures has not been assured. Due to the history of $Y$. pestis use as a biological weapon, combined with transmission through aerosols and high mortality rates, the United States designates Y. pestis as a Tier 1, Category A Select Agent. Due to the select agent classification restricts have been implemented for working with Y. pestis. To ensure proper containment of the agent, Y. pestis must be worked with in specialized facilities designed with engineering controls and personal protective equipment to prevent exposure to the pathogen. Additionally, precautions are taken to ensure proper handling and storage occurs to mitigate the potential release or misuse of the agent from occurring.

\section{Divergence from ancestral strains.}

Although infection with $Y$. pestis is one of the most deadly and rapidly progressing infections a human can contract, the closely related enteric pathogens Yersinia enterocolitica and Yersinia pseudotuberculosis are not as adept at killing humans as $Y$. pestis is [1, 27-29]. Both $Y$. pseudotuberculosis and Y. enterocolitica are food borne pathogens that cause a self-limiting gastroenteritis $[28,29]$. After ingesting either of these enteric pathogens, they cross the intestinal barrier and enter the intestinal lymph nodes (mesenteric) [30, 31]. The diseases caused by the enteric Yersinia are very similar due to divergence from a common ancestor and possession of many common virulence factors [27, 32, 33]. From Y. pseudotuberculosis, Y. pestis subsequently diverged, which included loss of two virulence factors important for enteric Yersinia pathogenesis 
(Invasin and YadA), and acquisition of two plasmids encoding murine toxin and the plasminogen activator (Pla) [2]. The divergence uniquely adapted Y. pestis to a vector borne lifestyle, while enhancing the ability of $Y$. pestis to disseminate and evade host innate immune responses.

$\underline{Y . ~ p e s t i s ~ i n f e c t i o n ~-~ a v o i d i n g ~ i n n a t e ~ i m m u n e ~ s y s t e m ~ m e d i a t e d ~ c l e a r a n c e . ~}$

Y. pestis maintains a hallmark non-inflammatory host environment up to $24 \mathrm{~h}$ postinfection. The innate immune system subsequently responds by causing massive expression of antimicrobial products and cytokines, referred to as a cytokine storm [34-36]. By the time an inflammatory response is mounted, $Y$. pestis has enacted a defense mechanism capable of neutralizing innate immune cells. Ultimately, necrosis of host tissues occurs without impacting $Y$. pestis replication, resulting in death of the host before an adaptive immune response is ever mounted [37]. The innate immune system is therefore the primary line of defense against $Y$. pestis.

How $Y$. pestis maintains an early non-inflammatory phase is a question that may hold the key to understanding Y. pestis disease progression. Innate immune cells are key to production of an inflammatory response to bacteria, and are thus likely being manipulated or subverted by $Y$. pestis to maintain the non-inflammatory phase [38]. From when Y. pestis was first identified as the etiological agent of plague, the $Y$. pestis field has attempted to better understand the ways in which Y. pestis interacts with the innate immune system [36, 37, 39]. Using a mouse model of infection, Y. pestis has been shown to interact primarily with neutrophils, macrophages, and to some extent with dendritic cells very early during infection, but these cells are not able to clear the infection [4043]. Due to the ability of $Y$. pestis to avoid clearance, research efforts have focused on understanding how $Y$. pestis interacts and avoids clearance by these innate immune cells [36, 41]. Possession of several virulence factors and modulation of key surface exposed antigens have allowed Y. pestis to subvert and directly combat innate immune responses to establish disease [33, $35,44-46]$.

\section{Changes in LPS allow evasion of inflammation.}

Toll like receptors (TLRs) are members of the host pattern recognition receptors (PRRs) that rapidly identify molecules produced by pathogens (referred to as pathogen-associated 
molecular patterns or PAMPS) leading to activation of the immune response [47]. Lipopolysaccharide (LPS) produced by Gram negative bacteria is typically recognized by TLR4 leading to activation of the NF-kB pathway triggering inflammatory cytokine and lipid mediator responses to coordinate multicellular responses to the infection [48]. However, all three pathogenic Yersinia are able to switch the LPS they display from a hexa-acylated TLR4-stimulatory form, to a tetra-acylated TLR4-non-stimulatory form with variability in lipid A structure [49]. Switching to the tetra-acylated non-stimulatory form allows for evasion of the innate immune response, demonstrated by data showing inhibition of LPS modification results in attenuation and clearance of $Y$. pestis due to ligation and signaling through a TLR4/MD2/ MyD88 dependent pathway $[48,50$, 51]. Y. pestis also lacks O-antigen, leaving the LPS core components exposed [49]. A recent study demonstrated this modification to be important for interaction with antigen presenting cells (APCs) and specifically for binding of the C-type lectin, CD209b (SIGNR1) to enhance dissemination of Y. pestis [52].

Ail contributes to complement resistance.

The enteric Yersinia have two important adhesins involved in virulence (Invasin and YadA) that Y. pestis does not have. A conserved adhesin, shared between all three pathogenic Yersinia, and important for virulence, is Ail [53-56]. Ail is an outer membrane protein (OMP) with greatest efficacy at $37^{\circ} \mathrm{C}$, when the $\mathrm{O}$ antigen of LPS is shed from the bacterial surface [57]. Exposure of Ail allows the bacterium to bind host $\mathrm{C} 4 \mathrm{~b}$ binding protein to inactive the complement component C3 convertase, protecting Yersinia from the complement cascade [45]. Additionally, Ail also enhances adherence and targeting of innate immune cells for Type 3 secretion (see below), thus diminishing recruitment of neutrophils and inhibiting inflammation $[53,55]$.

The Pla adhesin/protease contributes to dissemination.

The plasminogen activating protein (Pla), encoded on the PPCP1 plasmid, was acquired by $Y$. pestis and has been shown to aid in attachment to host cells $[33,58]$. Pla not only acts as an adhesin, it is also an omptin family of proteases, and has been shown to cleave host complement proteins and inhibit the function of plasminogen activator inhibitor -1 (PAI-1) [59]. Inhibition of PAI- 
1 blocks degradation of host extracellular matrix by matrix metalloproteases, limiting the breakdown of cellular junctions, and increasing the difficulty for host innate immune cells to infiltrate the site of infection and produce a pro-inflammatory response. A recent study by Banerjee et al. found that Pla also functions as a facilitator of T3SS targeting of alveolar macrophages, inhibits neutrophil infiltration into the lungs, and is important for resisting neutrophil mediated bacterial degradation [60, 61]. Together, Ail and Pla enhance targeting of innate immune cells and resist innate immune mediated killing of $Y$. pestis.

\section{The F1 Capsule protects against phagocytosis.}

In addition to Pla, Y. pestis also expresses a protein capsule called $\mathrm{F} 1$ that is not present in the enteric Yersinia [62]. F1 is produced by a modified chaperone usher system on the pMT plasmid $[63,64]$. The F1 capsule is temperature regulated and does not occur in the flea vector [65]. Expression of the $\mathrm{F} 1$ capsule is induced at $37^{\circ} \mathrm{C}$ upon transmission into the mammalian host. The F1 capsule blocks interactions with phagocytic cell receptors to contribute to inhibition of phagocytosis and protects the bacterium from antimicrobial peptides [66].

\section{The Type 3 Secretion System is essential for immune modulation.}

While the F1 capsule contributes to inhibiting uptake of $Y$. pestis, the Type 3 Secretion System (T3SS) encoded by the pCD1 plasmid is the primary virulence factor protecting Y. pestis from phagocytes [62]. Bacterial T3SS are molecular syringes that span both the inner and outer Gram negative bacterial membranes, as well as the mammalian plasma membrane [67]. Bacterial effector proteins are rapidly shuttled across all three membranes in an ATP dependent manner through the needle of the T3SS [68]. In addition to inhibiting phagocytosis, numerous studies have demonstrated the T3SS inhibits multiple arms of the innate immune responses [35, 36, 69-71]. The T3SS delivers seven bacterial Yersinia outer proteins (Yops) into the host cell cytosol (Table 1-1). Once inside host cells, these Yop effector proteins directly inhibit specific cellular signaling pathways [70]. Three effector proteins (YpkA, YopE, and YopT) target the host proteins Rac, Rho,

and other actin interacting proteins to directly inhibit cytoskeletal rearrangement, effectively inhibiting phagocytosis, motility, and other antimicrobial responses [72-76]. Each of these Yop 
effector proteins have different enzymatic activity. YpkA is a serine/threonine kinase, while YopE is a GTPase activating protein, and YopT is a cysteine protease $[72,74,76-80]$. Furthermore, YopH is capable of inhibiting cytoskeletal rearrangement through suppression of focal adhesion complex signaling, and also inhibiting calcium flux [73, 81-84]. YopJ targets the mitogen activating protein kinase (MAPK) signaling pathway to inhibit activation of nuclear factor $\mathrm{KB}$ (NF-kB), which regulates transcription of cytokines [71, 85-88]

Disruption of cytoskeletal rearrangement and MAPK signaling is important to inhibit antimicrobial and inflammatory responses, and greatly contributes to the non-inflammatory environment associated with plague. However, these activities can be recognized by host cells, resulting in activation of the inflammasome pathway and cell death $[89,90]$. Innate immune cell death due to activation of the inflammasome leads to production of inflammatory cytokines. To circumvent inflammasome activation due to disruption of the Rac and MAPK signaling by YopE and YopH, Y. pestis also delivers two Yop effector proteins capable of inhibiting inflammasome activation (YopM and YopK) [91-96]. Inflammasome activation is inhibited by the leucine rich repeat containing YopM through disruption of signaling by caspase-1 IQGAP1, and RSK1 [97-99]. Historically, the activity of YopK has been less well known. Previous studies have established that YopK acts as a regulator of Yop translocation into host cells, to limit inflammasome activation [92]. Recent work has shed further light on the activity of YopK, demonstrating that YopK is important for inhibiting guanylate binding protein mediated activation of the inflammasome, triggered by the T3SS translocon pore $[96,100]$. Together, the seven Ysc T3SS effector proteins cripple innate immune cell motility, phagocytosis, and the ability to raise warning signals through activation of the inflammasome.

While required for mammalian infection, the T3SS is not required in the flea and thus expression of the T3SS is tightly regulated, with temperature, $\mathrm{pH}$, and cation concentration all acting as signals for expression [101, 102]. The primary signal differentiating the flea and mammalian host is temperature. As such T3SS is low during growth at temperatures mimicking the flea $\left(<26^{\circ} \mathrm{C}\right)$ and increases when grown at mammalian temperatures $\left(34-37^{\circ} \mathrm{C}\right)$ T3SS expression also responds to calcium, magnesium, and iron concentrations [101, 103]. Cations are highly 
regulated in mammalian tissues, allowing pathogenic bacteria to use them as cues for expressing or repressing required virulence factors that are metabolically costly, or have the potential to errantly activate host defense mechanisms [104, 105].Importantly, since the T3SS (and other virulence factors) are not expressed in the flea, there is a transition period after deposition by flea feeding when $Y$. pestis is highly susceptible to being engulfed by phagocytic cells, such as macrophages and neutrophils [106-108]. While Y. pestis engulfed by neutrophils are quickly degraded, those bacteria phagocytosed by macrophages can survive and even subsequently replicate, eventually lysing the out of the macrophage. [107, 109]. The ability to survive and replicate within macrophages, but not within neutrophils, has led to the idea that $Y$. pestis uses macrophages early during infection as a way to subvert clearance by neutrophils. Additionally, exploitation of macrophages as a hideout would provide $Y$. pestis with the opportunity to upregulate expression of the T3SS and other virulence factors that allow the bacterium to better, combat neutrophil antimicrobial defenses.

\begin{tabular}{|c|c|c|c|c|}
\hline \multirow[t]{2}{*}{$\begin{array}{c}\text { Effector } \\
\text { Y. pestis/Enteric } \\
\end{array}$} & Activity & Verified Ho & Target & \multirow[t]{2}{*}{ Cellular Effect } \\
\hline & & $\begin{array}{l}\mathrm{M} \varphi \text { and other } \\
\text { cell types }\end{array}$ & Neutrophil & \\
\hline YpkA/YopO & $\begin{array}{c}\text { Serine/threonine } \\
\text { kinase }\end{array}$ & $\begin{array}{l}\text { VASP, WASP, } \\
\text { WIP, Gelsolin, } \\
\text { Gaq, EVL, } \\
\text { mDia1, INF2, } \\
\text { cofilin, RhoA, } \\
\text { Rac1, and } \\
\text { Rac2 }\end{array}$ & & $\begin{array}{l}\text { Inhibition of actin } \\
\text { cytoskeletal dynamics } \\
\text { contributing to } \\
\text { perturbance of } \\
\text { phagocytosis }\end{array}$ \\
\hline YopE & $\begin{array}{c}\text { GTPase } \\
\text { activating protein }\end{array}$ & $\begin{array}{l}\text { RhoA, Cdc42, } \\
\text { Rac2, RhoG }\end{array}$ & & $\begin{array}{l}\text { 1. Inhibition of actin } \\
\text { cytoskeletal dynamics } \\
\text { contributing to } \\
\text { perturbance of } \\
\text { phagocytosis } \\
\text { 2. Caspase-1 } \\
\text { inhibition resulting in } \\
\text { disruption of IL-1 } 1 \beta \text { and } \\
\text { IL-18 maturation event }\end{array}$ \\
\hline YopH & $\begin{array}{l}\text { Protein tyrosine } \\
\text { phosphatase }\end{array}$ & $\begin{array}{l}\text { FAK, p130cas, } \\
\text { paxillin, Fyb, } \\
\text { SKAP-HOM, } \\
\text { PRAM-1, SLP- } \\
\text { 76, Vav, } \\
\text { PLCy2, p85, }\end{array}$ & $\begin{array}{l}\text { SKAP- } \\
\text { HOM, } \\
\text { PRAM-1, } \\
\text { SLP-76 }\end{array}$ & $\begin{array}{l}\text { 1. Focal adhesion } \\
\text { complex disruption } \\
\text { culminating with } \\
\text { inhibition of } \\
\text { phagocytosis }\end{array}$ \\
\hline
\end{tabular}




\begin{tabular}{|c|c|c|c|}
\hline & & $\begin{array}{l}\text { Gab1, Gab2, } \\
\text { Lck, LAT }\end{array}$ & $\begin{array}{l}\text { 2. Pro-inflammatory } \\
\text { cytokine/MCP-1 } \\
\text { inhibition } \\
\text { 3. Calcium response } \\
\text { and ROS inhibition } \\
\text { 4. Inhibition of PI3K } \\
\text { and AKT pathways }\end{array}$ \\
\hline YopJ/YopP & $\begin{array}{l}\text { Acetyltransferase } \\
\text { Deubiquitinase } \\
\text { Cysteine } \\
\text { protease }\end{array}$ & $\begin{array}{c}\text { TRAF2, } \\
\text { TRAF6, IKBa, } \\
\text { MAPKKKs, } \\
\text { MAPKks, IKK } \beta \text {, } \\
\text { RICK, elF2 } \alpha\end{array}$ & $\begin{array}{l}\text { 1. Induces Caspase-1 } \\
\text { and apoptosis } \\
\text { induction }\end{array}$ \\
\hline Yop K & 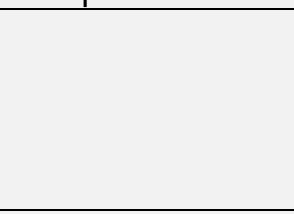 & Matrilin-2 & $\begin{array}{l}\text { 1. Regulates Yop } \\
\text { Translocation } \\
\text { 2. Regulates caspase } \\
\text { and apoptosis } \\
\text { activation }\end{array}$ \\
\hline YopM & $\begin{array}{l}\text { Leucine rich } \\
\text { repeat protein }\end{array}$ & $\begin{array}{c}\text { RSK, PRK, } \\
\text { Caspase-1, } \\
\text { IQGAP1 }\end{array}$ & $\begin{array}{l}\text { 1. Induces anti- } \\
\text { inflammatory cytokine } \\
\text { production, while } \\
\text { inhibiting pro- } \\
\text { inflammatory } \\
\text { 2. Caspase-1 and } \\
\text { apoptosis inhibition }\end{array}$ \\
\hline YopT & $\begin{array}{l}\text { Cysteine } \\
\text { protease }\end{array}$ & $\begin{array}{l}\text { RhoA, Rac1, } \\
\text { Cdc42, RhoG }\end{array}$ & $\begin{array}{l}\text { 1. NFkB inhibition } \\
\text { 2. Perturb actin } \\
\text { cytoskeleton }\end{array}$ \\
\hline
\end{tabular}

* denotes information specific to Y. pestis

Table adapted from [44]

Y. pestis creates a replicative niche within macrophages.

The idea that $Y$. pestis uses macrophages as a niche for evading neutrophil mediated destruction is supported by in vivo data pointing to the importance of macrophages for Y. pestis to fully establish an infection to cause disease. Chemical depletion of macrophages causes diminished dissemination from draining lymph nodes in a sphingosine-1-phosphate dependent manner [110]. The response to Y. pestis infection by macrophages is also important for controlling infection. In susceptible mice, it has been shown that the murine macrophages produce a non- 
inflammatory response (M2 phenotype), whereas in mice which are resistant to plague, the macrophages create an inflammatory response (M1 phenotype) better able to clear Y. pestis [111, 112]. Pre-treatment to induce an M1 phenotype in susceptible mice enhances the host response and is better at clearing $Y$. pestis infection [111] The requirement for monocytes/macrophages during in vivo infection and the evidence that pre-activated macrophages can better combat $Y$. pestis infection, supports the hypothesis that $Y$. pestis use naïve macrophages as a shelter while anti-host factor expression is upregulated. While the fact that $Y$. pestis is able to survive and replicate within macrophages is well accepted, how Y. pestis is able to subvert natural macrophage degradative mechanisms has not been well defined.

Macrophages are a host innate immune cell tasked with patrolling and controlling invasion by microorganisms such as bacteria. Macrophages engulf bacteria and encapsulate it within a vacuole termed a phagosome [47]. After engulfment, phagosomes are trafficked to a degradation specific pathway where they undergo modifications and fuse with another vesicle termed the lysosome [47]. Fusion with lysosomes results in formation of a phagolysosome where invading organisms are exposed to acidic conditions in which proteases are activated, resulting in degradation and elimination of microorganisms. To direct trafficking of phagosomes through the maturation process toward fusion with lysosomes, mammalian cells rely upon Rab GTPases [113, 114]. Early phagosomes associate with Rab5 and EEA1 [113, 115]. Through the maturation process, Rab5 is replaced by Rab7 to become a mature phagosome [113]. Fusion of lysomes with the phagosome further modifies the membrane so that Rab7 is lost and Rab9 is gained [113]. In addition to directing phagosome maturation, Rab GTPases are also involved in trafficking of other membranous vesicles throughout cells [113]. The importance of Rab GTPases in trafficking events within eukaryotic cells makes these host proteins the target of many bacterial pathogens.

Unlike other non-pathogenic bacteria, once phagocytosed, the $Y$. pestis phagosome does not progress through the phagosomal maturation pathway. Instead Y. pestis generates a vacuolar compartment termed the Yersinia containing vacuole (YCV) (Figure 1-1). YCVs do not appear to acidify over the course of macrophage infection and the $\mathrm{pH}$ in the YCV remains between 6.5 and 7.5. [107-109, 116-118]. Additionally, most YCVs fail to co-localize with Rab7, LAMP-1, and 
Cathepsin D, markers of mature phagosomes, and thus appear to avoid fusion with lysosomes [117]. Instead, large proportion of YCVs co-localize with Rab1b, 4a, and 11b early during biogenesis (Figure 1-1) $[118,119]$. Furthermore, recruitment of Rab1b and 4a to the YCV appears to be essential to avoid vacuolar acidification. $[118,119]$. Recruitment of Rab11b does not appear to be required to avoid acidification, but proceeds bacterial replication [119]. Sequestration of Rab11b on the YCV by Y. pestis disrupts global cellular recycling and leads to the induction of host cell autophagy, which has been linked to Yersinia intracellular replication [120,121]. Targeting of the host autophagy pathway has been postulated to serve as a source of membrane by which expansion of the YCV occurs to form a spacious vacuole. $[117,119]$. Within the spacious YCV, Y. pestis replicates and infected macrophages lyse between 12 and $15 \mathrm{~h}$ post infection [122]. To date the ability of $Y$. pestis to generate the YCV and replicate within macrophages has not been attributed to the function of a known virulence factor. Importantly, the T3SS is not required for intracellular survival. However, stress response genes such as those regulated by PhoPQ and the rip operon have been shown to enhance survival but not the biogenesis of the YCV [107, 123-126]. Therefore, there is currently a major gap in our understanding of how $Y$. pestis is able to avoid killing by macrophages.

While there is much still to be defined regarding how $Y$. pestis survives within macrophages, it is known that autophagy is required by $Y$. pestis for intracellular replication [117]. Autophagy is a catabolic mechanism utilized by cells to break down and recycle self or foreign structures [127]. When cellular components are damaged, or the cell is undergoing stress, autophagy is induced in order to break down the products and make available the liberated nutrients for repurposing $[127,128]$. Depending on the structure being degraded, autophagy is labeled differentially. When mitochondria are degraded, it is referred to as mitophagy, whereas when bacteria are the degradative target, it is referred to as xenophagy [129]. Autophagy functions by enveloping particles within a membrane bound compartment and a typical marker of autophagy is the formation of a double membrane structure [128]. Formation of the membranous compartment occurs as a cascade of activation signals, and depending on the type of autophagy, the steps in formation of autophagic vacuoles can differ. Generally, ULK1 associates with Beclin1, ATG 
proteins, and adaptor proteins such as p62 to begin forming the autophagosome [130]. Additional ATG proteins further elongation of the membrane by adding phosphatidylethanolamine to LC3-I, thus converting it to LC3-II and allowing for closure of the vesicle and sensitivity of the inner membrane to lysosomal degradation [131]. Rab proteins, such as Rab11 are important for autophagy, as they can direct recycling endosome membranes into the autophagy pathway to provide membrane for the autophagy expansion process [130]. To degrade the bacteria, syntaxin 17 associates with the autophagic membrane to facilitate fusion with lysosomes and create a structured termed an autolysosome $[131,132]$.

While autophagy is beneficial for eukaryotic cells undergoing stress, induction of autophagy is detrimental for bacteria which escape from phagosomes and enter into the host cell cytosol to replicate, such as Shigella flexneri or Burkholderia species [133, 134]. However, unlike bacteria which enter into the cytosol, those remaining within a vacuole can benefit from induction of autophagy, such as Anaplasma phagocytilium and Coxiella burnetti [135-138]. Moreover, for the vacuolar pathogen Y. pseudotuberculosis which is closely related to Y. pestis, LC3 is recruited to the vacuole and autophagy is important for replication within macrophages [120, 121]. Y. pestis has been observed co-localized to the autophagy marker LC3 and within a double membrane compartment, suggesting $Y$. pestis enters into the autophagy pathway and benefits from the nutrient liberation process similar to other intravacuolar pathogens [117].

\section{RNAi screens to identify host pathways important for intracellular survival.}

Our lab has proposed the hypothesis that $Y$. pestis targets host signaling pathways to avoid phagosome maturation to survive within macrophages. To date, conventional bacterial mutagenesis screens have been unable to identify bacterial factors required for YCV biogenesis. An alternative strategy to better understand the mechanisms used for YCV biogenesis is to identify the host factors required for $Y$. pestis intracellular survival. In order to better understand how other pathogens manipulate host processes, large scale RNA interference (RNAi) high throughput screening approaches have been used [139]. Utilization of RNAi has been useful for spotlighting the differences in host components required for vacuolar versus cytosolic pathogen survival and 
replication [139]. Moreover, other RNAi screens with Brucella abortus, Chlamydia trachomatis, and Salmonella typhimirium, have aided in better understanding the biological mechanisms these pathogens use to overcome degradation and to create a permissive intracellular environment for replication [140-144]. We used a similar strategy to identify three Rab GTPases (Rab1b, 4a, and 11b) as required for survival of $Y$. pestis within macrophages $[118,119]$. These studies highlight how targeting the host cell using RNAi can provide a better understanding of the mechanisms used by bacteria to survive within host cells. 


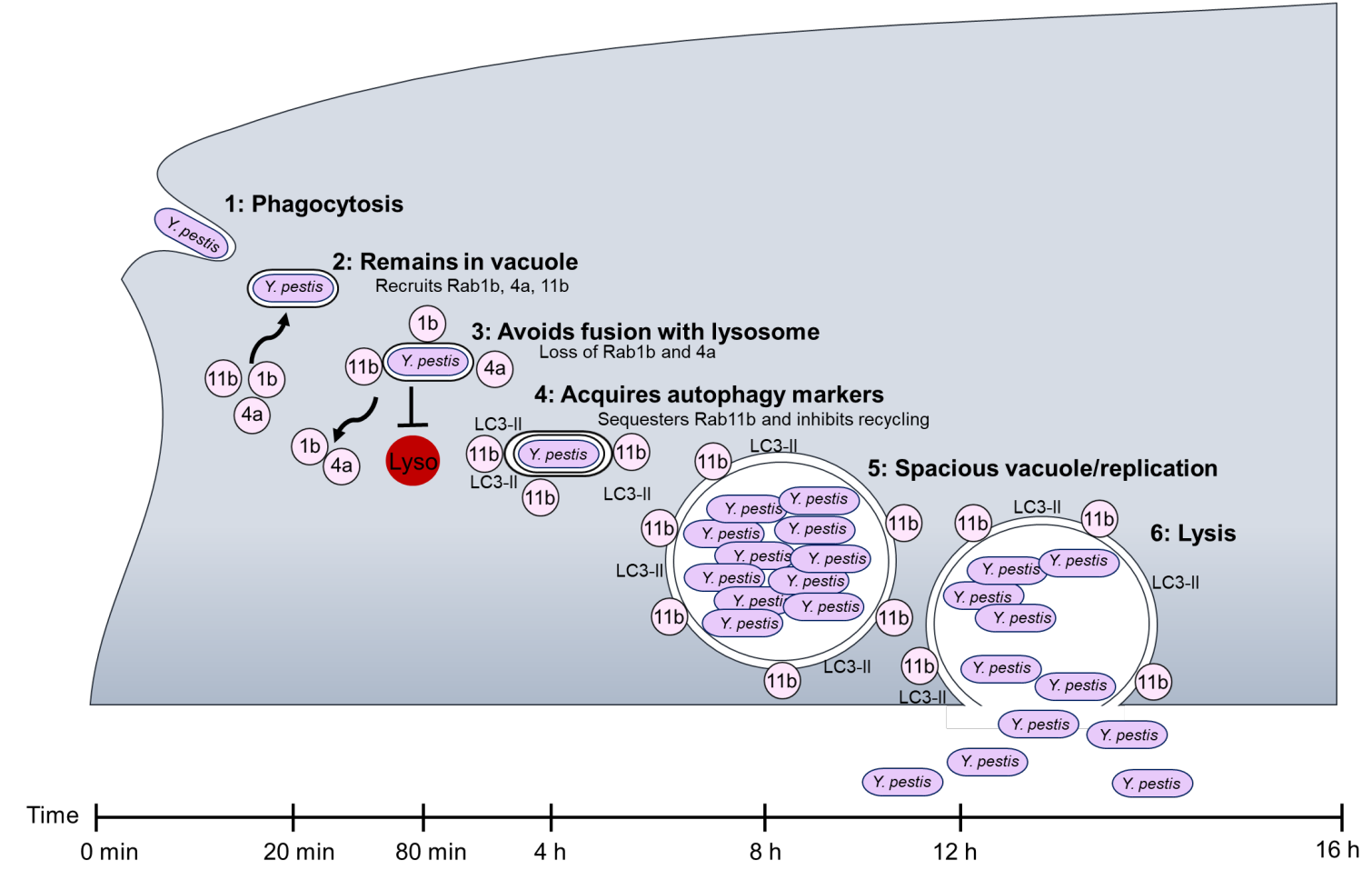

Figure 1-1: Y. pestis survival within macrophages.

Yersinia pestis is phagocytosed by macrophages, yet is not degraded. Phagosomal maturation and fusion with the lysosome does not occur, which is dependent on Rab1b. Instead Y. pestis acquires Rab1b, 4a, 11b and markers of autophagosomes early during infection, Rab1b and 4a are lost while Rab11b is retained and creates a spacious Yersinia containing vacuole wherein replication occurs. Finally Y. pestis lyses out of the infected macrophage between 12 and $18 \mathrm{~h}$ post-infection. Although it is well understood that $Y$. pestis is able to survive and replicate within macrophages, the precise mechanism of how Y. pestis diverts phagosome maturation from fusion with lysosomes remains unknown. 
Neutrophil antimicrobial defense mechanisms.

Macrophages are important for $Y$. pestis to disseminate and fully establish disease within the host $[52,145]$. Understanding what host components and pathways are used by Y. pestis to traffic within macrophages will improve our understanding of how the lysosomal degradative mechanism of macrophages is subverted and Y. pestis creates a niche for replication. Conversely, neutrophils are detrimental to the survival of $Y$. pestis within the host. In pneumonic plague, early recruitment of neutrophils to the lungs improves the survival rate of infected mice [146]. While $Y$. pestis is able to survive and replicate within macrophages, Y. pestis is not able to survive and replicate within neutrophils as observed for macrophages [147]. Even though macrophages and neutrophils are both innate immune cells that are able to destroy microorganisms, the antimicrobial mechanisms utilized by the two cell types are different in many ways.

Neutrophils are one of the most abundant leukocytes in the circulatory system and are vital for successful control of infections. Neutrophils comprise nearly $70 \%$ of the leukocytes within blood and were once thought to be short lived, with a life span of only a few hours, due to the monumental effort and toxicity combating the infection entails [148, 149]. However, this perceived characterization of neutrophil life cycle is being increasingly refuted by accumulating data pointing to neutrophils living longer than previously thought with the proper signals $[150,151]$. Neutrophils, along with other phagocytic cells such as dendritic cells (DCs) and macrophages, are continually surveilling for microbial invaders. Unlike DCs and macrophages, which differentiate and remain primarily as tissue resident cells, neutrophils primarily patrol in the blood stream, waiting for signals from endothelial, epithelial, or other phagocytic cells to indicate an infection or tissue damage has occurred in order to home to the tissues [151]. Upon activation, receptors are displayed on the neutrophil membrane to enhance homing abilities and allow for increased attachment to endothelial membranes. Increased display of integrins on the endothelium, and receptors/ligands on the neutrophil surface, allows neutrophils to slow, bind, and eventually stop in the swiftly moving bloodstream [151]. Tightly adhering to the endothelium, neutrophils begin the process of transmigration to enter into the underlying epithelial tissue [151]. 
Upon reaching the site of infection, neutrophils employ a multifaceted arsenal to neutralize the infection as swiftly as possible. Neutrophils use phagocytosis, release of granule contents into the phagosome or extracellular space, release of chromosomal content (neutrophil extracellular traps [NETs]), and release of reactive oxygen species (ROS) to negatively impact the survival and replication of invading microorganisms [152-157]. Although these mechanisms are detrimental to the microorganism, they are also destructive to the neutrophil and other nearby host cells. Therefore, neutrophils tightly regulate responses based on the pathogenic potential of the microorganism [149].

\section{Phagocytosis.}

Phagocytosis by neutrophils is a very rapid event, occurring within seconds, compared to macrophages which require minutes to complete phagocytosis [158]. Upon phagocytosis of the microorganism, neutrophils do not traffic the phagosome in the same way as macrophages. Instead, neutrophil phagosomes remain at a neutral $\mathrm{pH}(\sim \mathrm{pH} 7)$, and do not fuse with lysosomes to degrade microorganisms [158]. Instead, neutrophils rely upon fusion with compartments called granules and a potent oxidative burst to kill bacteria [149, 153, 158, 159].

\section{Phagosome granule fusion and ROS response.}

Of the mechanisms neutrophils possess to combat microbial infections, production of reactive oxygen species is one of the most effective yet self-destructive, damaging DNA, RNA, lipids, and proteins [160-162]. Therefore, production of ROS is a tightly controlled mechanism that relies upon other defensive measures to occur before ROS can be produced [153]. To make ROS, neutrophils assemble several subunits to create the catalytically active unit known as the NADPH oxidase [163-165]. The subunits are the membrane bound cytochrome b558, which has two components, $\mathrm{p} 22^{\text {phox }}$ and gp91 ${ }^{\text {phox }}$, and cytosolic p47 phox $, \mathrm{p} 40^{\text {phox }}, \mathrm{p} 67^{\text {phox }}$, and Rac2 [165]. The membrane bound cytochrome b558 is stored in neutrophil granules, the phagosome must undergo fusion with granules containing cytochrome $b_{558}$ for the respiratory burst to occur. Inhibition of granule fusion results in decreased phagosomal ROS production [153]. Production of ROS is more effective at killing invading organisms within phagosomes due to the small area in which it is 
contained aiding in concentrated exposure. However, some microorganisms cannot be phagocytosed [166]. To overcome the inability to phagocytose a microorganism, neutrophils shift targeting of toxic antimicrobials away from phagosomal delivery, to extracellular release $[155,167$, 168]. Neutrophils are able to release not only ROS into the extracellular milieu, but granule contents as well $[167,168]$.

\section{Degranulation.}

Production of ROS is a potent product that interferes with many different targets within the invading organism, and the action of ROS is complemented by the potent antimicrobials packed into the granules of neutrophils. Similar to other granulocytes, such as basophils, eosinophils, and mast cells, neutrophils form preformed granules during maturation [167]. The granule cargo are formed and packaged for rapid release to respond to a stimulus as quickly as possible. Granules are not homogeneous and there are four different types of granules formed by neutrophils, with additional subtypes (Figure 1-2) [167]. There is a hierarchical order in which the granules are formed and also in how they are released [167]. While many studies have focused on understanding how these four types of granules are differentially regulated for release, the exact mechanisms are still under investigation [148, 169].

Primary (Azurophilic) granules.

The first granule to form, yet the last to be released, are primary granules also known as azurophilic granules [167]. The name azurophilic granule comes from the azurocidin contained within, which acts as a chemoattractant and increases vascular permeability [167]. Azurophilic granules contain the most potent antimicrobials formed by neutrophils such as lysozyme, defensins, bactericidal/permeability increasing protein (BPI), and myeloperoxidase (MPO) [167]. While most of the antimicrobial peptides function to directly interact with microorganism membranes, MPO reacts with $\mathrm{H}_{2} \mathrm{O}_{2}$ produced by $\mathrm{NADPH}$ oxidase to cause additional toxicity through formation of hypochlorous acid, in addition to others [167]. The toxicity produced by azurophilic granule components can cause significant collateral damage to surrounding host tissue. Therefore, release is tightly regulated and requires additional stimulus for release than for the other 
granules. Azurophilic granules are the final granule to be released, as they are held for direct interaction with microorganisms.

\section{Secondary (Specific) granules.}

Through the process of myelopoiesis, the second granule type to be formed are secondary granules, otherwise termed specific granules [167]. Specific granules have a duel role, as they are important for direct combat with microorganisms, due to the antimicrobials they contain, while also functioning for neutrophil movement across cell barriers via breakdown of extracellular matrix [167]. The antimicrobials within specific granules include; natural resistance- associate macrophage protein 1 (Nramp-1), lactoferrin, and neutrophil gelatinase associated lipocalin (NGAL) [167]. The antimicrobials within specific granules predominantly function to inhibit microbial survival and growth through sequestration of metals [167]. However, there are some that are also capable of interacting with microbial components to impact membrane integrity [167].

\section{Tertiary (Gelatinase) granules.}

As with specific granules, gelatinase granules also have cargo to assist in movement of neutrophils across vasculature and through tissues [167]. One of the proteins contained within gelatinase granules is matrix metalloprotease-9 (MMP-9), referred to as gelatinase, from which the granule name was derived [167]. The activity of MMPs is inhibited within granules, as it is stored as a pro-form which undergoes cleavage upon granule release to activate the enzyme [167]. In comparison to azurophilic and specific granules, gelatinase granules are released earlier and with

milder stimulus, such as the bacterial peptide mimetic, $\mathrm{N}$-formylmethionyl-leucyl-phenylalanine (fMLF) [167].

\section{Secretory vesicles.}

Although secretory vesicles are not strictly granules, but more so plasma membrane derived vesicles, they are still classified as one of the four granule subtypes neutrophils possess [167]. Secretory vesicles are the last of the four granules to be formed and require the least stimulus to trigger release with fMLF sufficient to cause substantial release [153]. The function of secretory vesicle release is to prime neutrophils to respond to additional signals that an infection is occurring, 
and begin moving toward the site of infection [167]. Through release of secretory vesicles, membrane receptors are displayed, such as formyl peptide receptors, the $\beta_{2}$ integrin, complement receptor 3 [CR3], in addition to others [167]. The main cytoplasmic component of secretory vesicles is albumin, due to the endocytic nature of formation [167]. Surface expression of secretory vesicle receptors decreases over time without further stimulus, as receptors are removed through endocytosis of the plasma membrane [167].

As mentioned previously, neutrophil granules are released with specific order mediated through tightly regulated pathways. For degranulation to occur, $\mathrm{Ca}^{2+}$ must be mobilized from intracellular stores, along with rearrangement of the actin cytoskeleton $[155,161,170]$. A barrier of cortical actin exists below the plasma membrane of neutrophils, impeding the release of granules $[169,171]$. For granules to be released, this actin barrier must be broken down to provide access to the plasma membrane for fusion $[148,171]$. Secretory vesicles, gelatinase and specific granules are distal to the plasma membrane, residing closer to the nucleus and require a cytoskeleton dependent shuttling to reach the plasma membrane [167]. Conversely, a subset of azurophilic granules are located just below the plasma membrane and do not require shuttling via the cytoskeleton from further cytoplasmic recesses [171]. Exocytosis is coordinated through a complex network of regulatory proteins consisting of Rac2, RhoA, Rab27a, Gem-interacting protein (GMIP), and other cytoskeletal interacting proteins $[154,155,167,171,172]$. Through fine-tuned regulation of the degranulation process, neutrophils rapidly respond to signals of invasion and coordinate movement to the site of infection, judiciously releasing the possessed weaponry either into the phagosomal compartment for minimal collateral damage, or into the extracellular space when phagocytosis fails to contain the infection $[155,158,173]$. 


\begin{tabular}{|c|c|c|c|}
\hline \multirow[b]{2}{*}{ Secretory Vesicles } & \multirow[b]{2}{*}{ Gelatinase Granules } & \multirow[b]{2}{*}{ Specific Granules } & \multirow[b]{2}{*}{ nhilic Granules } \\
\hline & & & \\
\hline $\begin{array}{c}\text { Contain } \\
\text { Transmembrane } \\
\text { Proteins/Receptors: } \\
\text { fPR1, Mac-1, CD16, } \\
\text { CR1, CXCR2 }\end{array}$ & $\begin{array}{l}\text { Contain Proteases: } \\
\text { Gelatinase B (MMP-9), } \\
\text { ADAM9, lysozyme }\end{array}$ & $\begin{array}{l}\text { Contain Proteases and } \\
\text { Antimicrobials: } \\
\text { Lactoferrin, Lysozyme, } \\
\text { Lipocalin (NGAL), } \\
\text { MMP-8, etc. }\end{array}$ & $\begin{array}{l}\text { Contain Antimicrobials: } \\
\text { MPO, Azurocidin, } \\
\text { Cathepsin G, Elastase, } \\
\text { Proteinase 3, Defensins }\end{array}$ \\
\hline $\begin{array}{l}\text { Chemotaxis signaling } \\
\text { and motility of PMNs }\end{array}$ & $\begin{array}{l}\text { Tissue remodeling and } \\
\text { extravascular motility }\end{array}$ & $\begin{array}{c}\text { Nutritional immunity } \\
- \text { sequestration of } \\
\text { metals }\end{array}$ & $\begin{array}{l}\text { Potent antimicrobial } \\
\text { components directly } \\
\text { target pathogens }\end{array}$ \\
\hline $\begin{array}{l}\text { Marker of } \\
\text { granule release: } \\
\text { Release of Albumin }\end{array}$ & $\begin{array}{c}\text { Marker of } \\
\text { granule release: } \\
\text { Release of MMP-9 }\end{array}$ & $\begin{array}{c}\text { Marker of } \\
\text { granule release: } \\
\text { Surface expression } \\
\text { of CD66b }\end{array}$ & $\begin{array}{c}\text { Marker of } \\
\text { granule release: } \\
\text { Surface expression } \\
\text { of CD63 }\end{array}$ \\
\hline Blood & $\begin{array}{l}\text { Exit blood/ } \\
\because \because \text { Enter tissue }\end{array}$ & $\begin{array}{c}\text { Enter site } \\
: \because \text { of infection }\end{array}$ & $\begin{array}{l}\text { Site of } \\
\text { infection }\end{array}$ \\
\hline
\end{tabular}

\section{Figure 1-2: Neutrophil Granules.}

Neutrophils have four types of granules that each contain different cargo. Granules are released in response to stimuli in a graded manner. The first granule to be released are specific vesicles that function to increase the number of receptors on the plasma membrane. The second granule released are gelatinase granules that release proteases to increase the ability of neutrophils to move out of the vasculature and into the target tissue. Second to last to be released are specific granules which contain antimicrobials that function to target bacteria or sequester metals known as nutritional immunity. While azurophilic granules are the last to be released, they contain the most potent antimicrobials. 
Neutrophil extracellular traps (NETs).

Failure of phagocytosis, ROS, and degranulation to contain the infection cause neutrophils to rely on decondensing and release of DNA to entrap microorganisms $[149,156,165,174,175]$. Historically release of neutrophil extracellular traps (NETs) has been viewed as a last ditch defense mechanism for protecting the host as release of NETs was thought to kill neutrophils. Classical NETosis, in which the neutrophil granule and nuclear membranes are compromised, renders neutrophils no longer viable and is a death pathway independent of apoptosis [175, 176]. However, recent studies now suggest that neutrophils have a second mechanism which differs from suicidal NETosis, where the nucleic material is released in a membrane enclosed vacuole that allows the neutrophil to continue to fight against the infection, referred to as vital NETosis [177].

Nuclear decondensation and membrane perforation requires participation from several neutrophil pathways. In order for NETosis to occur, granule membranes must be perforated, along with the nuclear and plasma membranes $[175,176]$. Membrane rupture must occur in order for neutrophil elastase (NE), myeloperoxidase (MPO), and peptidylarginine deiminase 4 (PAD4) to enter into the nucleus, where each functions to modify DNA packaging through histone modifications [174-176]. In addition to the requirement of NE, MPO, and PAD-4, neutrophils also require activation of protein kinase $C$ (PKC), NADPH oxidase, and the lipoxygenase pathway in order to rupture the cellular membranes and release nucleic material $[178,179]$. As a result of granule and plasma membrane rupture, DNA from the neutrophil is adorned with granule components, such as NE, MPO, and others, in addition to cytosolic proteins such as the metal sequestration protein, calprotectin (SA100A8/9) [157]. Together, nucleic acid entraps the invading organism, while granule contents continue a defensive program in an attempt to starve and kill the invader. After expulsion of genomic material, granules contents, and formation of ROS, with nothing left to defend the host, depleted neutrophils must rely upon incoming neutrophils and other phagocytic cells to carry forth the tide of defense. 
Neutrophils modulate the immune response through release of Leukotriene $B_{4}$.

Neutrophils produce an assortment of cytokines and chemokines as signals to other cells to relay the urgency and severity of response required. Of the inflammatory mediators possessed by neutrophils, leukotriene $B_{4}\left(L_{T B}\right)$ is the first to be released, and one of the most potent chemoattractants $[180,181]$. LTB 4 is a lipid derived from membrane arachidonic acid (AA) through a series of enzymatic processes. To produce $\mathrm{LTB}_{4}, \mathrm{AA}$ is cleave from membranes, such as the nuclear envelope, plasma membrane, or membrane bound vesicles in the cytoplasm [182, 183]. Cleavage of $A A$ from membranes occurs via activation of phospholipase $A_{2}\left(P L A_{2}\right)$, thereby increasing the free AA concentration within the cell [184]. In order to convert AA into other products, 5 lipoxygenase (5-LO) must be activated and translocated to a membrane enriched in 5 lipoxygenase activating protein (FLAP) [185, 186].

Regulation of 5-LO activity is multi-faceted (Figure 1-3). Phosphoylation by ERK1/2 and p38 have been indicated to increase 5-LO activity, while phosphorylation through PKA acts in an opposing manner to decrease 5-LO activity [187-191]. Binding of AA to 5-LO blocks interaction with PKA and limits the ability of PKA to phosphorylate 5-LO and thereby decrease 5-LO activity [190]. In addition to modification through phosphorylation, 5-LO also binds to $\mathrm{Ca}^{2+}$ and increases the affinity of 5-LO for phosphytidyl choline (PC), driving 5-LO toward association with PC enriched membranes where FLAP also resides [190, 192]. While coactisin like protein (CLP), a cytoskeleton interacting protein, is also an interacting partner with 5-LO, the exact role CLP plays in the activity of 5-LO is not fully apparent [186]. Upon activation and translocation of 5-LO, a secondary cascade of enzymatic reactions occur. The $5-\mathrm{LO}$ product $5-\mathrm{HpETE}$ is converted to $\mathrm{LTA}_{4}$ and subsequently converted to $\mathrm{LTB}_{4}$ through the action of $\mathrm{LTA}_{4}$ hydrolase $(\mathrm{LTA} 4 \mathrm{H})$ [190]. Once $\mathrm{LTB}_{4}$ has been produced, release into the extracellular space is currently proposed to be mediated by exosomes $[193,194]$. This hypothesis is supported by data showing LTB$_{4}$ does not freely diffuse out of cells, and that there is a rate limiting step to release [195]. Additionally, free $\mathrm{LTB}_{4}$ has a shorter half-life in comparison to exosome encased $\mathrm{LTB}_{4}$, which ties into the chemoattractant nature of $\mathrm{LTB}_{4}$ to signal over long distances [194]. 


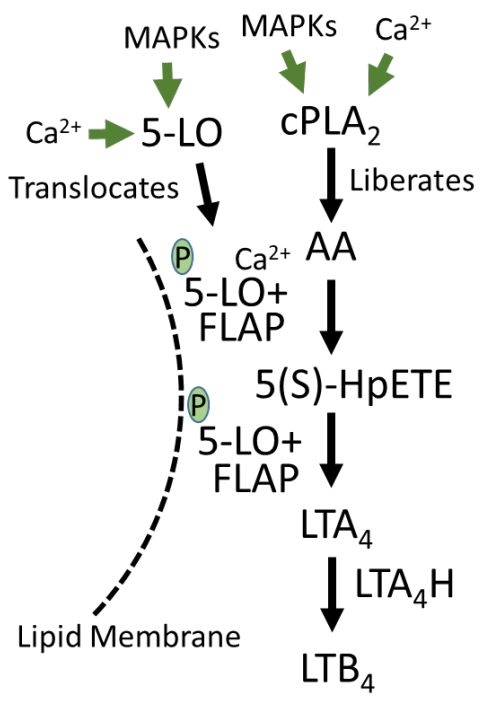

\section{Figure 1-3: Synthesis pathway for $\mathrm{LTB}_{4}$.}

Human neutrophils produce $\mathrm{LTB}_{4}$ to a greater extent than most other innate immune cells. $\mathrm{LTB}_{4}$ is a potent inducer of chemotaxis, but is also able to modulate immune cell antimicrobial activities. Production of $\mathrm{LTB}_{4}$ is a multistep process that can involve integration of multiple signaling pathways. Receptor sensing of PAMPs triggers activation of the MAPK pathway in addition to release of $\mathrm{Ca}^{2+}$ from intracellular stores. Phosphorylation of 5-LO and/or association with $\mathrm{Ca}^{2+}$ triggers translocation of 5-LO to a lipid membrane (i.e. nucleus or lipidosome) while also activating $\mathrm{CPLA}_{2}$ to liberate arachidonic acid (AA). Through the combined activity of 5-LO and 5-LO activating protein (FLAP), AA is modified to form $5(\mathrm{~S})$-HpETE and further processed by 5 -LO to form LTA 4 . Ultimately, $\mathrm{LTA}_{4}$ is processed by $\mathrm{LTA}_{4}$ hydrolase to form $\mathrm{LTB}_{4}$. 
While $\mathrm{LTB}_{4}$ is a potent chemoattractant, it is also capable of increasing the antimicrobial activity of multiple immune cells [196-199]. $\mathrm{LTB}_{4}$ has been shown to impact neutrophil ROS production, degranulation, NETosis, and increase the efficacy of phagosomal degradation [200202]. In addition to the impact of $\mathrm{LTB}_{4}$ on neutrophils, $\mathrm{LTB}_{4}$ also modulates cytokine production in macrophages, as well as impacts dendritic cell (DC) presentation to T cells and cytokine release $[200,203,204]$. The influence of $\mathrm{LTB}_{4}$ on DC presentation links the impact on innate immune cells to influencing of adaptive immune responses as well [196, 197, 204-206] [207]. Taken together, $\mathrm{LTB}_{4}$ plays a crucial role in successful clearance of microbial infections and has been demonstrated to be important for clearing infections by pathogens such as Klebsiella pneumonia, Borrelia burgdorferi, and also for fungal and parasitic infections [198, 201-203, 208-210].

\section{Caveats when working with neutrophils}

It is important to note that during in vitro assays, how neutrophils are handled can impact observed phenotypes more than other host cells. For example, the process of adhering neutrophils to substrates can modify the activation state and trigger release of granules that would not occur without additional stimulation when kept in suspension. Simple differences, such as using suspension versus adherent neutrophils, and the charge or composition of the surface adhered to, can modulate other responses such as ROS production and phagocytosis [211-214]. Interestingly, even signaling pathways can change pending the cues a neutrophil receives. In suspension, neutrophils signal through the MAPKs ERK and p38, yet upon adherence, JNK is then able to relay in the phosphorylation cascade [215-217]. The differential responses must be kept in mind when thinking about how neutrophils are signaling and responding to microbial infections.

Considerations also need to be made when comparing responses between mice and humans. For example, human TLR4 is more sensitive to LPS than the mouse counterpart, resulting in a stronger inflammatory response in humans versus mice [218]. Not only is TLR4 different between mice and humans, mice also have additional TLRs not encoded by humans [218]. Other receptors also differ between mice and humans, such as the formyl peptide receptors (FPR) and complement receptors $(C R)[218,219]$. In a very generalized summary, mouse receptors have less 
affinity/activity than human receptor counterparts [218]. Furthermore, inducible nitric oxide synthase activation requires different cytokine signals in mouse cells versus human [220]. Not only are there differences in receptor sensitivity and activation, there are also differences in cellular content. Human azurophilic granules have bacterial permeability inducing protein and defensins that are not part of the cargo in mouse azurophilic granules [218]. Moreover, although mouse azurophilic granules have been shown to contain myeloperoxidase (MPO), human azurophilic granules have up to 10x more MPO than murine azurophilic granules [218]. Therefore, what is observed during in vitro studies using mouse neutrophils may not always translate to human neutrophils and vice versa, highlight the need to verify results with mouse cells in primary human cells.

Inhibition of neutrophil antimicrobial responses by $Y$. pestis.

With the array of antimicrobial mechanisms neutrophils can deploy to defend against infection by microorganisms, they are one of the most vital innate immune cells for controlling infection. During Y. pestis pneumonic infection, there is an early non-inflammatory phase, where neutrophils are not recruited into the lungs until 36-48h post infection [221]. Similarly, the inflammatory cytokines (KC and MIP-2) also do not increase until the same time frame [146, 222]. Concomitant with the arrival of neutrophils to the site of infection, the non-inflammatory phase ends and an inflammatory response is mounted, resulting in necrosis and ultimately death of the host $[36,223]$. If neutrophils are artificially recruited to the site of infection earlier than normal, a decrease in bacterial burden and an increase in overall host survival are observed in a mouse model of infection [146], indicating that maintenance of this early non-inflammatory environment is important to establish infection.

During colonization by $Y$. pestis, resident neutrophils have been shown to be a primary target for T3SS effector translocation $[42,43]$. These interactions allow $Y$. pestis to inhibit several neutrophil antimicrobial responses (Figure 1-4) [166, 224]. YpkA, YopE, YopH, and YopT inhibit cytoskeleton rearrangements via interactions with Rac2 and RhoA to suppress phagocytic uptake of $Y$. pestis [72-74, 76-82, 166, 225]. Targeting of Rac2, a component required for activation of the 
NADPH oxidase, by YopE also contributes to inhibiting ROS, but requires complementary actions by YopJ inhibition of MAPK signaling and YopH targeting of the focal adhesion complex to fully inhibit ROS by neutrophils [226, 227]. Finally, YopJ inhibition of MAPK signaling also inhibits release of IL-8 by neutrophils, though additional, yet to be identified, Yops are also required for full inhibition of release [224]. However, the pathways regulating granule release and LTB 4 production in neutrophils have not been previously examined in the context of $Y$. pestis infection. The pathways regulating granule release and $\mathrm{LTB}_{4}$ production are also regulated by the same host factors known to be targeted by the Yops (e.g. MAPK pathway, $\mathrm{Ca}^{2+}$ flux, and Rac/Rho signaling) $[154,155,162$, $188,190,192,228]$. Because the pathways are common between these neutrophil antimicrobial responses, the likelihood that these processes are also inhibited by Y. pestis during infection, and contribute to the ability of the bacterium to subvert killing by neutrophils, is increased. Moreover, while much of our understanding of the host targets for individual Yops has come from studies in macrophages, data showing direct interaction between specific Yops and their predicted host targets in neutrophils lags behind. Understanding the mechanisms of how Y. pestis inhibits neutrophil responses early during infection will shed light on how $Y$. pestis maintains an early noninflammatory environment beneficial to $Y$. pestis survival. 


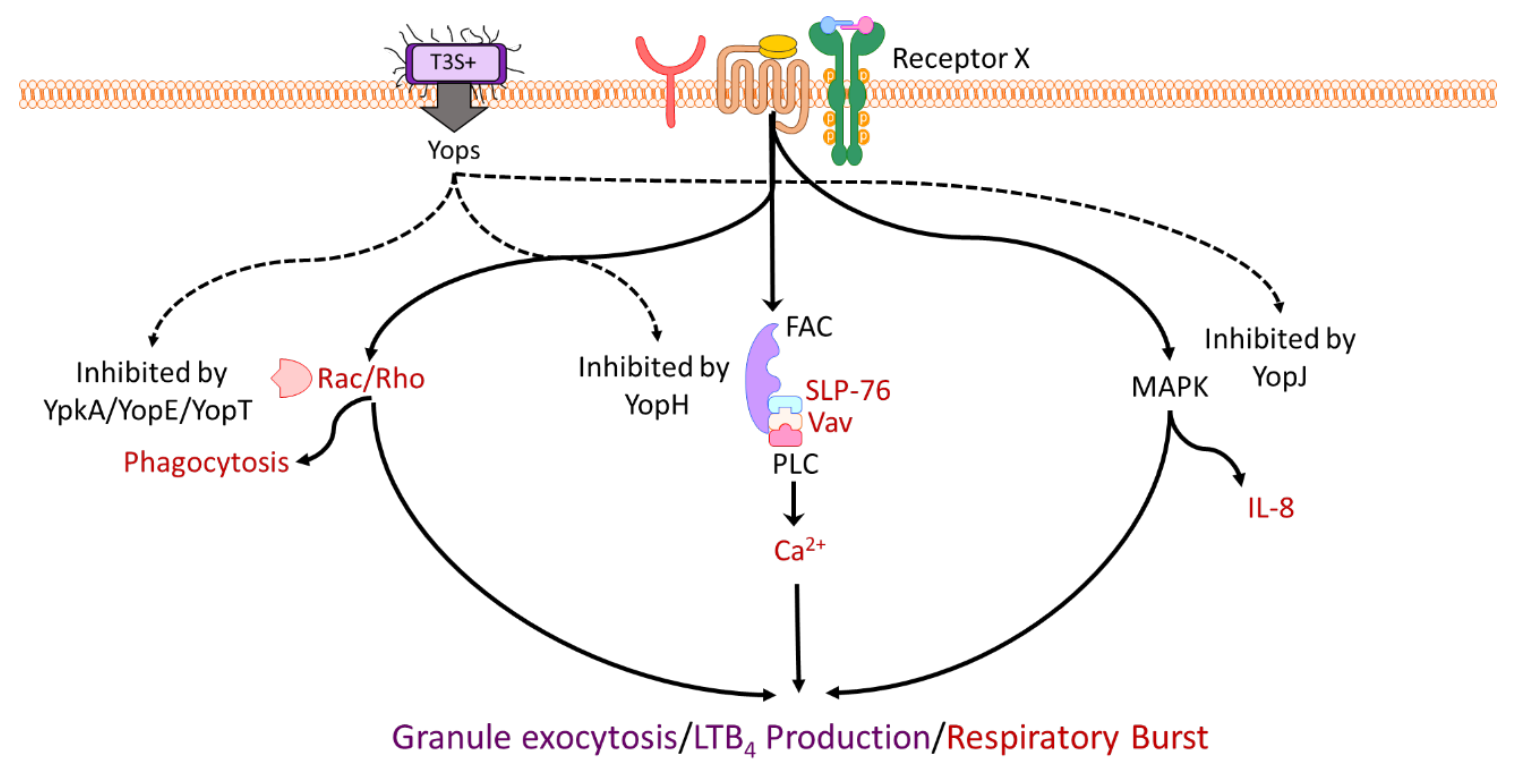

Figure-1-4: Y. pestis Yop Effector Protein Impact on Neutrophil Antimicrobial Responses.

Injection of bacterial effector proteins into the cytosol of neutrophils allows for interaction with host cell signaling pathways. YopJ is known to inhibit release of IL-8 from neutrophils, but interaction with MAPKs in neutrophils has not been demonstrated. YopH acts through interaction/inhibition of FAC proteins to inhibit intracellular $\mathrm{Ca}^{2+}$ flux that is required for multiple downstream effects in neutrophils, such as degranulation, ROS production, and release of $\mathrm{LTB}_{4}$ and. YpkA, YopE, and YopT modulate actin cytoskeletal rearrangement pathways through targeting of Rac and RhoA, inhibiting phagocytosis and the ability of neutrophils to move. While the role of Yop effector proteins have been assessed (red) for inhibition of phagocytosis, the respiratory burst, and IL-8 release, the impact on granule exocytosis and LTB4 production has not been determined (purple). 


\section{RESEARCH OBJECTIVES}

Interactions with host innate immune cells dictate whether $Y$. pestis is successfully cleared by the infected host. Two of the most essential host phagocytic cells responsible for clearing infections are macrophages and neutrophils. An inherent interplay exists between these cells, as signaling from one cell type influences the antimicrobial properties of the other through release of cytokines and chemokines. The goal of this work is to better understand how Y. pestis survives the initial onslaught of phagocytic cells both intra- and extracellularly, while maintaining an environment that does not incite inflammation until late in the infection process. Y. pestis is able to survive and replicate within macrophages, yet known virulence factors, such as the T3SS are not required. Many pathogens manipulate Rab trafficking to establish a replicative niche within macrophages. Therefore, I hypothesize that Y. pestis modulates host Rab GTPases to avoid lysosomal degradation, establish the YCV, and buy time to increase expression of the T3SS to subsequently target neutrophils. Furthermore, whether $Y$. pestis inhibits release of granules and LTB 4 has never been evaluated, but I hypothesize that $Y$. pestis actively inhibits neutrophil granule and LTB4 release using the T3SS effector proteins. Together, my overarching hypothesis is that $Y$. pestis manipulates Rab GTPases to survive within macrophages, buying time to express the T3SS, which is then used to inhibit neutrophil antimicrobial capabilities including granule and $\mathrm{LTB}_{4}$ release, ultimately maintaining the early non-inflammatory environment observed during Y. pestis infection.

To test this hypothesis, I have formulated specific questions with briefly described research objectives to answer those questions outlined here:

1. How does Y. pestis avoid phagolysosomal mediated killing within macrophages? To address this question, I:

a. Completed an RNAi screen to survey Rab GTPases required for intracellular macrophage survival.

i. Identified Rab GTPases important for Y. pestis intracellular survival which are recruited or excluded from the YCV in order to modulate vesicular trafficking. 
2. Does Y. pestis modulate the neutrophil antimicrobial response in order to maintain an early non-inflammatory host environment?

In an attempt to answer this question, I:

a. Measured whether the four neutrophil granules are released in response to $Y$. pestis.

i. Evaluated whether granule release is inhibited by the Ysc T3SS.

ii. Determined which Yop effector protein(s) mediate inhibition of granule release.

b. Measured $\mathrm{LTB}_{4}$ production and release in response to $Y$. pestis infection of neutrophils

i. Determined if the T3SS inhibits release of $\mathrm{LTB}_{4}$ from human neutrophils.

ii. Determined which Yop effector protein(s) are mediating inhibition of LTB 4 release and the host pathways which are targeted.

The subsequent chapters present data to improve our understanding of how Y. pestis evades degradation by macrophages and neutrophils to survive, establish a replicative niche, and avoid an early inflammatory response. 
CHAPTER 2

MATERIALS AND METHODS 


\section{Cell Culture, Bacterial Strains, and Plasmids}

RAW264.7 macrophages were obtained from ATCC and cultured in Dulbecco modified Eagle medium (DMEM) containing $100 \mathrm{mM}$ glucose plus $10 \%$ fetal bovine serum (FBS) (Biowest) at $37^{\circ} \mathrm{C}$ and $5 \% \mathrm{CO}_{2}$. For plasmid transfection of RAW264.7 macrophages, $0.5 \mu \mathrm{g}$ of plasmid (EGFP-Rab) was transfected using JetPrime (Polyplus) as described by the manufacturers.

Y. pestis CO92 pCD1(-) [5] was cultivated at $26^{\circ} \mathrm{C}$ in Difco brain heart infusion broth (Becton, Dickinson, and Co.). E. coli K-12 DH5a (New England Biolabs) was cultivated at $37^{\circ} \mathrm{C}$ in LuriaBertani broth (Miller) (Becton, Dickinson, and Co.). Bioluminescent (CO92LuXPtolC) [5] or fluorescent (pGEN222::mCherry) [118] derivative strains were used as indicated for infections. Y. pestis was inactivated by incubating bacteria with $2.5 \%$ paraformaldehyde (PFA) for $30 \mathrm{~min}$ at room temperature as previously described [118] Extracellular Y. pestis and E. coli were killed with 16 $\mu \mathrm{g} / \mathrm{ml}$ gentamicin for $1 \mathrm{~h}$, followed by maintenance in $2 \mu \mathrm{g} / \mathrm{ml}$ gentamicin. 
Table 2-1: Bacterial Strains

\begin{tabular}{|c|c|c|c|}
\hline Descriptive name & Genotype & $\begin{array}{l}\text { Strain } \\
\text { Number }\end{array}$ & Source \\
\hline CO92 T3+ & $\begin{array}{l}\text { CO92 pCD1(+) } \operatorname{pgm}^{(+)}, \mathrm{pMT}^{(+)}, \mathrm{pst}^{(+)} \\
\text {Luxp }_{c y s z K}\end{array}$ & MBLYP043 & [5] \\
\hline C092 T3- & CO92 pCD1(-) pgm $^{(+)}, \mathrm{pMT}^{(+)}, \mathrm{pst}^{(+)}$ & YPA035 & [5] \\
\hline CO92 T3+ & MBLYP043 pgm( ${ }^{(-)}$ & YPA143 & This work \\
\hline C092 T3- & $\begin{array}{l}\text { CO92 pCD1 } 1^{(-)}, \mathrm{pgm}^{(+)}, \mathrm{pMT}^{(+)}, \mathrm{pst}^{(+)} \\
\text {LuXPtolC }\end{array}$ & YPA050 & [122] \\
\hline KIM T3+ & KIM1001 pCD1(+), pgm $^{(-)}, \mathrm{pMT}^{(+)}, \mathrm{pst}^{(+)}$ & JG150A & [229] \\
\hline KIM T3- & KIM1001 pCD1(-), pgm ${ }^{(-)}$ & JG152B & This work \\
\hline KIM T3E- & 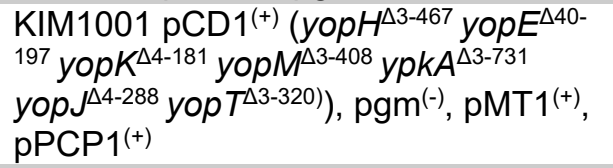 & JG714 & This work \\
\hline$+\mathrm{A}$ & JG917::+ypkA & JG730 & [229] \\
\hline$+\mathrm{E}$ & JG917::+yopE & JG733 & [229] \\
\hline$+\mathrm{H}$ & JG917::+уорH & JG734 & [229] \\
\hline$+\mathrm{J}$ & JG917::+yopJ & JG735 & [229] \\
\hline$+\mathrm{K}$ & JG917::+yopK & JG736 & [229] \\
\hline$+\mathrm{M}$ & JG917::+уорM & JG732 & [229] \\
\hline$+\mathrm{T}$ & JG917::+yopT & JG708 & [229] \\
\hline$\Delta \mathrm{A}$ & JG150A $\Delta y p k A$ & JG593 & [229] \\
\hline$\Delta \mathrm{E}$ & JG150A $\triangle y o p E$ & JG517 & [229] \\
\hline$\Delta \mathrm{H}$ & JG150A $\triangle$ yopH & JG589 & [229] \\
\hline$\Delta \mathrm{J}$ & JG150A $\Delta y o p J$ & JG525 & [229] \\
\hline$\Delta \mathrm{K}$ & JG150A $\triangle$ уорK & JG523 & [229] \\
\hline$\Delta \mathrm{M}$ & JG150A $\triangle$ yopM & JG583 & [229] \\
\hline$\Delta \mathrm{T}$ & JG150A $\triangle y o p T$ & JG713 & [229] \\
\hline $\begin{array}{l}\text { CO92 T3-::pGEN222- } \\
\text { mCherry }{ }_{k}\end{array}$ & $\begin{array}{l}\text { CO92 pCD1 } 1^{(-)}, \text {pgm }^{(+)}, \mathrm{pMT}^{(+)}, \text {pst }^{(+)} \text {, } \\
\text { pGEN222::mCherry }\end{array}$ & YPA127 & {$[5,119]$} \\
\hline $\begin{array}{l}\text { E. coli::pGEN222- } \\
\text { mCherry }\end{array}$ & DH5a- pGEN222::mCherry & LOU123 & [119] \\
\hline KIM D-19 & $\begin{array}{l}\text { KIM D-19 pCD1(+) }{ }^{(+)} \operatorname{pgm}^{(-)}, \mathrm{pMT}^{(+)} \text {, } \\
\mathrm{pst}^{(+)} \text {, LuXPtolC }\end{array}$ & YPA119 & [119] \\
\hline $\begin{array}{l}\text { Y. pseudotuberculosis } \\
\text { T3+ }\end{array}$ & IP32593 pYV ${ }^{(+)}$ & MBL256 & [2] \\
\hline $\begin{array}{l}\text { Y. pseudotuberculosis } \\
\text { T3- }\end{array}$ & IP32593 pYV(-) & LOU016 & [2] \\
\hline Y. enterocolitica T3+ & JB580 pYV ${ }^{(+)}$ & MBL016 & [230] \\
\hline Y. enterocolitica T3- & JB580 pYV(-) & MBL077 & [230] \\
\hline
\end{tabular}

\section{$\underline{\text { Rab GTPase RNAi Screen }}$}

The Rab GTPases RNAi screen was performed as previously published [119]. Small interfering RNAs (siRNAs) from the Silencer siRNA mouse genome library v3 (Ambion) were used to forward 
transfect RAW264.7 macrophages. Three siRNAs targeting each gene were pooled together for the screen. siRNAs were suspended in $20 \mu$ I Opti-MEM (final concentration of $1 \mu \mathrm{M}$ ) and mixed with $10 \mu \mathrm{l}$ of $0.03 \%$ (vol/vol) Lipofectamine RNAiMax/Opti-MEM. The mixture was added to each well of a 96-well, white, flat bottom, plate (Greiner Bio One). Scrambled siRNA (negative control; $n$ $=3$ ) and Cop $\beta 1$ siRNA (positive control; $n=3$ ) were used as negative and positive controls, respectively for transfection efficiency and plate-to-plate variation. The plates were incubated at room temperature for $10 \mathrm{~min}$, before adding $80 \mu$ of DMEM plus $10 \%$ FBS (HyClone) at $1 \times 10^{4}$ RAW264.7 macrophages. The cells were incubated for $48 \mathrm{~h}$ prior to infection with $\mathrm{Y}$. pestis CO92LuXPtolc $\mathrm{pCD}^{(-)}$(multiplicity of infection [MOI] of 10). The infection was synchronized using centrifugation $(200 \mathrm{x} \mathrm{g}$ ) for $5 \mathrm{~min}$. Extracellular bacteria were killed with gentamicin as described above after incubation for $20 \mathrm{~min}$. Bioluminescence at $20 \mathrm{~min}$ and $2 \mathrm{~h}$ and $10 \mathrm{~h}$ was used to quantify intracellular bacteria post-infection using a Synergy 4 plate reader (BioTek; 1 -s read with sensitivity set at 150). Cell viability was determined using Alamar Blue (Life Technologies) after the $10 \mathrm{~h}$ read by adding $10 \mu \mathrm{l}$ of Alamar Blue to each well and incubating for $2 \mathrm{~h}$ at $37^{\circ} \mathrm{C}$ and $5 \% \mathrm{CO} 2$. Fluorescence (excitation wavelength, $560 \mathrm{~nm}$; emission wavelength, $600 \mathrm{~nm}$ ) was determined using a Synergy 4 plate reader. The average of the scrambled-siRNA control wells were used as controls for comparing to. Using the formula: 1 - (3 X (SD Cop $\beta 1$ RLU - SD scrambled RLU)/(AVG scrambled RLU - AVG Cop $\beta 1$ RLU)) where SD is the standard deviation and AVG is the average, a $Z$ factor $\left(Z^{\prime}\right)$ was calculated from each plate. Plates with $Z^{\prime}=0$ of $<0.3$ were repeated. The following formula: (siRNA RLU/AVG Copß1 RLU)/(AVG scrambled RLU/AVG Copß1 RLU) was used to normalize intracellular survival for each plate. Screen selection criteria was set at $\geq 50 \%$ inhibition of $Y$. pestis survival with $\leq 50 \%$ cytotoxicity as measured by alamar blue. Selection criteria was set to $\geq 50 \%$ inhibition of $Y$. pestis survival and $\leq 50 \%$ cytotoxicity base on three independent siRNA tests for a validation screen.

\section{Fluorescence Confocal Microscopy}

For confocal microscopy, cells were fixed to coverslips with $2.5 \%$ paraformaldehyde for $30 \mathrm{~min}$. All coverslips were mounted with Prolong Gold with DAPI (Life Technologies) and imaged on a Zeiss 
LSM 710 laser confocal microscope. Co-localization was determined using the COLOC module in IMARIS 8.0 (Bitplane).

\section{$\underline{\text { Neutrophil Studies: Bacterial Growth Conditions }}$}

Bacterial strains used in these studies are listed in Table 2-1. Prior to infection, Y. pestis was cultured for 15 to $18 \mathrm{~h}$ at $26^{\circ} \mathrm{C}$ in Difco brain heart infusion (BHI) broth (BD Biosciences) with aeration. Cultures were diluted 1:10 in fresh $\mathrm{BHI}$ broth containing $20 \mathrm{mM} \mathrm{MgCl} 2$ and $20 \mathrm{mM} \mathrm{Na}-$ oxalate and cultured at $37^{\circ} \mathrm{C}$ for $2 \mathrm{~h}$ with aeration to induce expression of the T3SS. Bacteria were centrifuged and re-suspended in LPS-free Krebs-Ringer phosphate buffer $(\mathrm{pH} 7.2)$ containing $0.2 \%$ dextrose (Krebs) buffer for infection. Y. pestis was killed using either 1\% PFA for 30 min, or heat killed by incubating $Y$. pestis at $60^{\circ} \mathrm{C}$ for $30 \mathrm{~min}$. The killed bacteria were pelleted and re-suspended in Krebs buffer prior to infection.

\section{$\underline{\text { Human Neutrophil Isolation }}$}

Use of human neutrophils was approved by the University of Louisville's Institutional Review Board (IRB) guidelines (approval no. 96.0191). Neutrophils were isolated from peripheral blood of healthy, medication-free donors as described previously [231]. Neutrophil isolations yielded $>95 \%$ purity with $>97 \%$ viability by Trypan blue exclusion staining and were used within $1 \mathrm{~h}$ of isolation. For RNAseq analysis, negative selection using EasySep ${ }^{\mathrm{TM}}$ antibody mediated magnetic separation was used to remove cells other than neutrophils, yielding highly pure (>99\%) neutrophils as previously described [232]

\section{$\underline{\text { Human Neutrophil Infection }}$}

Throughout these studies, all infections were performed in suspension as opposed to using adherent neutrophils. Cells in suspension were used primarily for two reasons: 1) The activation state of cells in suspension is closer to patrolling blood neutrophils, and. 2) All four granules can be assayed for release, unlike adherent neutrophils where the adherence process stimulates release of secretory vesicles. An important consideration for studies performed with cells in suspension is that the infections cannot be synchronized by centrifugation, and thus require higher 
MOls to ensure bacterial interactions with host cells, compared with assays using adherent cells that can be synchronized by centrifugation.

Neutrophils $\left(4 \times 10^{6}\right.$ cells; for Western blotting, $8 \times 10^{6}$ cells were used) were re-suspended in Krebs buffer or RPMI $+5 \%$ BSA and, where indicated, incubated at room temperature (RT) for 30 min with $1 \mu \mathrm{M}$ latrunculin A (catalog [cat.] no. 428021; Sigma), $20 \mu \mathrm{M}$ U0126 (cat. no. 70970; Cayman), 50 nM LY293111 (cat. no. 10009768; Cayman), or $3 \mu \mathrm{M}$ (5Z)-7-oxozeaenol (cat. no. 17459; Cayman). Neutrophils were infected at a multiplicity of infection (MOI) of 10 or 100 and incubated for $30 \mathrm{~min}, 1 \mathrm{~h}$ or $3 \mathrm{~h}$ in a $37^{\circ} \mathrm{C}$ water bath with gentle agitation. Coinfections were performed at a final MOI of 100 (50 for each strain), and bacteria were mixed together prior to adding to the cells. For secretory vesicles, specific and azurophilic granule exocytosis, the increases in plasma membrane expression of CD35, CD66b and CD63, respectively, were measured by flow cytometry as previously described [153]. To measure release of calprotectin, or $\mathrm{LTB}_{4}$, separate samples were centrifuged, and cell-free supernatants were transferred to new tubes containing Halt phosphatase and protease inhibitor cocktail (cat. no. 78442; Thermo Fisher Scientific), except for calprotectin samples and stored at $-80^{\circ} \mathrm{C}$. Calprotectin samples where neutrophils were treated for $3 \mathrm{~h}$ with PMA were unfrozen and mixed with a quantity of $Y$. pestis or $E$. coli equivalent to what would have been present during the neutrophil infection for the volume used, to assess degradation of calprotectin by proteolytic activity (e.g. $4 \times 10^{8} \mathrm{CFU}$ were added to $4 \times 10^{6}$ neutrophils to achieve an MOI of 100 in $1 \mathrm{~mL}$. For $100 \mathrm{uLs}$ of the PMA stimulated supernatant, $4 \times 10^{7} \mathrm{CFU}$ were used during the $3 \mathrm{~h}$ no cell incubation.) The samples were incubated at $37^{\circ} \mathrm{C}$ for $3 \mathrm{~h}$.

\section{Measurment of Exocytosis by Flow Cytometry and ELISA}

Neutrophils were stained with fluorescein isothiocyanate (FITC)-labeled anti-CD63 (cat. no. 215040; Ancell), FITC-labeled anti-CD66b (cat. no. 305104; BioLegend), or PE labeled CD35 as markers for azurophilic, specific granules, or secretory vesicles, respectively. As antibody isotype controls, neutrophils were separately labeled with FITC-labeled anti-lgM (cat. no. 401108; BioLegend) or FITC-labeled anti-lgG1 (cat. no. 400108; BioLegend) on ice for 45 min before washing with FTA buffer (BD Biosciences) plus $0.05 \%$ sodium azide and fixing with $1 \%$ paraformaldehyde (PFA). Mean cellular fluorescence intensity (MCF) was measured using a 
fluorescence-activated cell sorting (FACS) Aria flow cytometer (BD Biosciences) with isotype control values subtracted as previously described [233]. Human Calprotectin (cat. No 439707; BioLegend), mouse calprotectin (cat. no ab263885; Abcam) and LTB4 (cat. no. 520111; Cayman) levels were measured by enzyme-linked immunosorbent assay (ELISA) following the manufacturer's protocols.

\section{Chemotaxis Assay}

Supernatants from infected neutrophils were filtered using a $0.2 \mu \mathrm{m}$ syringe filter to generate conditioned supernatants. Naive neutrophils $\left(1 \times 10^{6} \mathrm{cells} / \mathrm{ml}\right)$ were loaded into the upper chamber of a 24-well Transwell plate (Corning). The lower chambers were filled with Krebs buffer, $100 \mathrm{nM}$ fMLF (Sigma), or the conditioned supernatants. After incubation for $30 \mathrm{~min}$ at $37^{\circ} \mathrm{C}$, neutrophils that migrated from the upper chamber to the lower side of the Transwell membranes were fixed and stained with Hema 3 (ThermoFisher) and counted by microscopy as described previously [234].

\section{Western Blotting}

After 30 min of infection, cell pellets were obtained by centrifugation $(6,000 \times$ gfor $30 \mathrm{~s})$. Pellets were lysed using ice-cold lysis buffer (20 mM Tris- $\mathrm{HCl}$ [pH 7.5], $150 \mathrm{mM} \mathrm{NaCl}, 1 \%$ [vol/vol] Triton X-100, 0.5\% [vol/vol] Nonidet P-40, 20 mM NaF, 20 mM NaVO3, 1 mM EDTA, 1mM EGTA, 5mM phenylmethylsulfonyl fluoride [PMSF], 2mM diisopropylfluorophosphate [DFP], $21 \mu \mathrm{g} / \mathrm{ml}$ aprotinin, and $5 \mu \mathrm{g} / \mathrm{ml}$ leupeptin). Lysates were mixed with Laemmli loading buffer and boiled for $10 \mathrm{~min}$ prior to snap cooling. Lysates were run on a $10 \%$ SDS-PAGE gel and immunoblotted with antibodies to phospho-ERK1/2, total ERK1/2, phospho-p38 MAPK, or total p38 MAPK (Cell Signaling) diluted 1:2,000 in $10 \mathrm{ml}$ of Tris-buffered saline plus $0.1 \%$ Tween 20 (TBST) plus $5 \%$ bovine serum albumin (BSA). The appropriate secondary antibodies were used at 1:50,000 (cat. no. A9169; SigmaAldrich; cat. no. 31430; ThermoFisher Scientific). SuperSignal West Femto maximum-sensitivity substrate (cat. no. 34095; ThermoFisher Scientific) was used to detect antigen-antibody binding. Densitometry was performed using ImageJ software to quantify bands, normalized using the total protein form. 
$\underline{\text { In vivo Infection }}$

C57BL6 wild type mice aged 6-12 weeks were infected intranasally with either WT Y. pestis Lux $\mathrm{xyszk}_{\text {or }}$ Y. pestis pCD1(-)LuX $\mathrm{x}_{\mathrm{cyszk}}$ at $1 \times 10^{4} \mathrm{CFU}$. Bacterial growth was monitored via CFU enumeration and BALF from the lungs was harvested at $12,24,48 \mathrm{~h} \mathrm{PI}$, or at the time of euthanasia.

\section{$\underline{\text { Statistics }}$}

Degranulation and $\mathrm{LTB}_{4}$ data are the mean of five biological independent experiments. Phosphorylation data are the mean of three biological independent experiments. For all, neutrophils were harvested from both male and female donors and infections were performed on different days. Where appropriate, one-way analysis of variance (ANOVA) with Dunnett's or Sidak's post-test, as indicated in individual figure legends, was used for statistical analysis and performed using Prism 8 (GraphPad). Unless noted, data are shown as the means \pm standard error of the mean (SEM). For microscopy, each experiment analyzed at least $100 \mathrm{YCVs}$, and power analyses were performed post-hoc to ensure that appropriate sample sizes were analyzed. P values were calculated using one-way analysis of variance (ANOVA) using GraphPad Prism software. 
CHAPTER 3

IDENTIFICATION OF RAB GTPASES CONTRIBUTING TO Y. PESTIS INTRACELLULAR SURVIVAL 


\section{INTRODUCTION}

Rab GTPases are proteins that shepherd intracellular vesicles to and from various locations within the cell. Over 70 Rab proteins are encoded within human cells and have specific subcellular localization [114, 235]. Activity of Rab GTPases requires several accessory proteins to assist Rab proteins in cycling between a membrane-GTP bound active state, to a cytosolic GDPbound inactive state [236]. GAP disassociation inhibitors (GDI) bind to inactive GDP-bound Rab GTPases and remove them from membranes [236]. GDIs bind the lipid anchoring prenyl group to protect the hydrophobic tail, removing the Rab from the membrane, and returns the Rab to the membrane of origin $[236,237]$. Switching between an inactive GDP bound state and active GTP bound state is carried out through the action of GDP/GTP exchange factor (GEF) and the reversal from GTP to GDP is enhanced by GTP activation proteins (GAP) [236]. With the assistance of GAPs, GEFs, and GDIs, Rabs carry out membrane trafficking duties in association with other interacting proteins to systematically direct vesicle activities. Rabs associate with vesicles in all stages of vesicle life, from forming vesicles through membrane budding, to coating, transporting, uncoating/tethering, and fusing to the target membrane [238].

During phagosome maturation, a sequence of Rab proteins associate and disassociate from the vacuole. The early endosome ( which early phagsomes can be classified as) associate with Rab5 [113]. As the vacuole matures and progresses to fusion with lysosomes, Rab5 dissociates from the vacuole membrane. Following the loss of Rab5, Rab7 is recruited and is required for fusion to lysosomes [113]. The elegant sequence of Rab recruitment and removal is essential to vacuole maturation. If disrupted, the endosome fails to mature, and the endosomal cargo is not exposed to enzymes like Cathepsin D or the phagosome may not undergo acidification through action of vATPases, ultimately resulting in endosomal cargo not being degraded [113].

Direct recruitment or exclusion of Rab proteins to or from the pathogen containing vacuole is a common way in which pathogens modulate phagosome maturation and remodel the vacuole into a niche for replication [239-245]. Hijacking host vesicular trafficking is a mechanism commonly exploited by intracellular pathogens and is an area of interest for better understanding the ways in which pathogens manipulate host cells to establish intracellular replicative niches. The exact 
mechanisms pathogens use to target host factors are multitudinous and often carried out through the action of bacterial effector proteins. Some pathogens encode relatively few effector proteins, while others such as Legionella pneumophila encode more than 300 [246]. The effector proteins are delivered into the host cell through bacterially encoded secretion systems that act as molecular delivery systems from the cytosol of the bacteria, through the membrane, and deposit the substrate either outside of the bacterial cell, or within the host cell $[67,247]$. Several different types of secretion systems exist, and many of them are able to deliver products to promote bacterial virulence. The T3SS, T4SS, and T6SS are capable of bridging both bacterial membranes and the host cell plasma/phagosomal membrane to directly deliver effector proteins into target cells [67].

Understanding the mechanisms Y. pestis uses to survive and replicate within macrophages is important for developing potential therapeutic strategies to prevent bacterial survival and replication. Previous studies have established that $Y$. pestis resides within a membrane bound compartment for the duration of the time $Y$. pestis is within macrophages $[108,117,248]$. Within this membranous compartment, $Y$. pestis is not exposed to acidic and degradative factors as would be expected for a non-pathogenic bacterium [117]. Instead, Y. pestis avoids fusion with the lysosome and the $\mathrm{pH}$ within the YCV remains between $\mathrm{pH} 6.5$ and 7.5 for the duration of the time within macrophages [109, 117-119].

We have shown that avoidance of acidification is dependent on the host factors Rab1b and Rab4a $[118,119]$. Inhibition of fusion with the lysosome is an active process, as paraformaldehydefixed Y. pestis traffics to the lysosome and is degraded within an acidic vacuole [118]. Intriguingly, neither Rab5 or EEA1, nor Rab7 or Cathepsin D, markers for early endosomes and mature phagolysosomes, respectively, have been found in association with the YCV [117]. Whether the lack of Rab5 indicates an alternative mechanism of entry into the cell, or if Rab5 association and disassociation from the YCV is too rapid to detect, has not been determined. More recently, we have shown that Rab1b, $4 a$, and $11 b$ are recruited to the YCV $[118,119]$, and $60-80 \%$ of YCVs eventually develop into autophagosomes, highlighted by association with LC3-II and acquisition of a second membrane [117]. 
Autophagy is a cellular recycling process that breaks down vacuolar contents to reuse the nutrients liberated [128]. Furthermore, autophagy is a mechanism to inhibit the growth of pathogens residing within the cytosol [249]. However there is evidence that autophagy can be beneficial to intracellular pathogens [250]. Specifically evidence from Salmonella suggests that autophagy may present a way for pathogens which reside in vacuoles to acquire nutrients [244, 251]. Rab11b, which interacts with the autophagy pathway, also appears to be recruited to the $Y C V$, and there is evidence that it is the bacteria within autophagosomes which are able to replicate within macrophages $[117,130]$

Because Rab GTPases are commonly targeted by bacterial pathogens to subvert lysosomal degradation by macrophages, and three Rab GTPases have already been shown to be essential for $Y$. pestis survival within macrophages, I was interested to know whether additional host Rab GTPases were essential for intracellular survival and biogenesis of the YCV. Using an RNAi approach, I was able to show that six Rab GTPases in addition to Rab1b, 4a, and 11b are required for $Y$. pestis to survive in macrophages. Moreover, I defined the relationship between the YCV and three of the new Rab targets during Y. pestis infection of mouse macrophages.

\section{$\underline{\text { RESULTS }}$}

RNAi screen identifies Rab GTPases required for $Y$. pestis survival within macrophages.

Rab GTPases are a common target for bacterial pathogens to subvert host cell vesicular trafficking that would otherwise be detrimental to the survival and/or replication of the pathogen [252]. To identify additional Rab proteins required by Y. pestis for macrophage intracellular survival, a Rab GTPase specific RNAi screen was performed in a mouse macrophage cell line. A pooled siRNA approach was used, similar to that reported by Connor et al. [119]. Briefly, three siRNA targeting a single Rab protein were pooled and transfected into RAW264.7 macrophages. The macrophages were then infected with a bioluminescent Y. pestis (Y. pestis CO92 pCD1(-) LuXPtolC) and intracellular survival was monitored as a function of bioluminescence, and host cell survival was monitored by Alamar blue staining. For this primary screen, positive cutoff criteria was set as $50 \%$ reduction in intracellular $Y$. pestis survival compared to scrambled siRNA, and less than $50 \%$ 
decrease in cell viability. Of the 39 Rab GTPases screened, RNAi of 13 Rabs met this criteria (Figure 3-1). Importantly, the three Rab GTPases previously shown as required for $Y$. pestis intracellular survival, Rab1b, 4a, and 11b, were among these hits [119].

While siRNA technology has come a long way, one potential problem is false positive results from off target inhibition of unattended targets, which can be exacerbated in pooled siRNA screens. A common technique to increase confidence in RNAi screens, and to reduce the likelihood of false positives, is to validate primary hits from a pooled siRNA screen with a secondary screen in which the pooled siRNAs are deconvoluted (i.e. each siRNA from the pool is individually screened for phenotypes). In this scenario, if a phenotype is due to an off target artifact, it should only occur with one of the three siRNAs. If the phenotype is validated with two or more of the siRNAs, it is then significantly more likely to be a true positive hit and is less likely to be an off target artifact. Using this approach, RAW264.7 cells were transfected with individual siRNAs (three siRNAs for each gene) for the 13 Rab GTPases identified in the primary pooled siRNA screen cells were then infected with Y. pestis CO92 pCD1 $1^{(-)}$LuXPtolc or Y. pestis KIMD19 pCD1(+) LuXPtolc., Genes in which at least three of the six individual infections resulted in $\geq 50 \%$ reduction of intracellular survival were considered validated hits.

From the 13 primary hits, eight Rab GTPases were validated as required for Y. pestis intracellular survival within macrophages (Figure 3-2). Moreover, as I expected, there were not significant differences in intracellular survival between a strain caring the pCD1 plasmid (encoding the T3SS) and one lacking PCD1, supporting previous data that the T3SS is not required for YCV biogenesis [117].

\section{Rab GTPases are differentially localized to the YCV.}

To influence vesicular trafficking of the phagosome, many intracellular pathogens recruit specific Rab proteins to the vacuole membrane [252]. Each Rab GTPase has a unique role in maintaining membrane traffic and must directly insert into the target membrane to function [114]. The coordinated interactions between the membrane, Rab GTPase, Rab interacting partners, and other host structures, such as the cytoskeleton, cause membrane shuttle flow from one cellular location to another via vesicle transport [115]. Disruption of Rab membrane association/localization 
alters membrane trafficking, and can lead to errant trafficking events. For example, if membrane targeted to the golgi errantly localizes with Rab11b, instead of Rab1b, the vesicle would traffic as a part of the recycling pathway, not to the golgi apparatus. Therefore, I sought to determine if $Y$. pestis recruited the validated Rab GTPases to the YCV during infection as a way to alter phagosome maturation. We previously characterized localization of Rab4a and Rab11b [119], which left localization of six Rab GTPase hits as unknowns. I prioritized four of the six remaining Rab proteins by degree of change in intracellular survival observed in the validation screen, giving higher priority to those whose RNAi resulted in greatest inhibition of $Y$. pestis intracellular survival $(2 b>23>13>22 a>40 b>20)$. I then also considered the functional categories of the individual Rab proteins. The Rab proteins identified from the screen generally fall into one of four functional categories: recycling (Rab23, 13, and 22a), sorting endosome (Rab20), secretory (Rab40b), or retrograde $($ Rab2b) trafficking. Three of the top four hits fall in the recycling pathway, which we have already established as being important for YCV biogenesis [119]. Therefore, to expand our analysis to include additional pathways, I chose to examine Rab20 over Rab23 because interactions with the sorting endosome is likely the earliest step in the YCV biogenesis process, and the role of the sorting complex in the context of $Y$. pestis infection is not as well understood. Therefore, Rab2b, 13, 22a, and 20 were chosen for further characterization. 


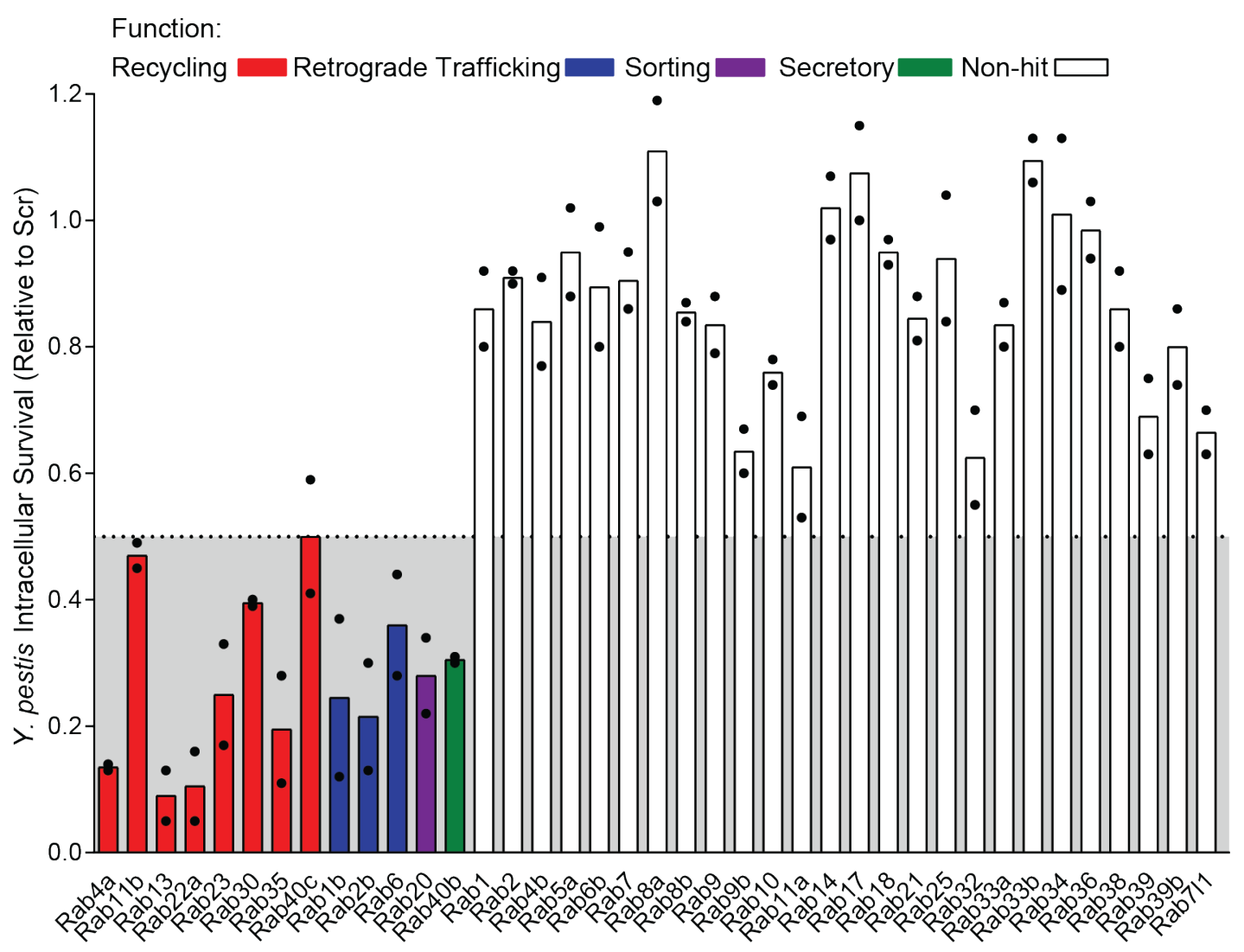

Figure-3-1: Rab proteins identified as essential for Y. pestis intracellular survival.

RAW 264.7 macrophages were transfected with three siRNAs targeting 39 different Rab genes. Forty-eight hours after transfection, cells were infected with Y. pestis CO92 pCD1(-) LuXPtolC (MOI of 10), and intracellular bacterial numbers were determined by bioluminescence (RLU) at $2 \mathrm{~h}$ or 10 h post-infection. Scrambled (Scr) siRNA was used as a negative control. Data is shown as the mean percent of intracellular bioluminescence at $10 \mathrm{~h}$ post-infection compared to $2 \mathrm{~h}$ post-infection for two independent replicates. Values for which $Y$. pestis intracellular growth was inhibited $\geq 50 \%$ compared to scramble are highlighted in the gray shaded area. Predicted trafficking pathways each identified Rab belongs to is indicated by color: red = recycling; blue = retrograde trafficking; purple =sorting; green = the secretory pathway; white = did not meet cutoff criteria. 
To test whether Y. pestis recruits these Rab proteins to the YCV, RAW264.7 macrophages overexpressing GFP-tagged Rab proteins were infected with Y. pestis CO92 pGEN222::mCherry (Yp) or E. coli K-12 pGEN::mCherry $(\mathrm{Ec})$, the latter bacterium is non-pathogenic and readily degraded by macrophages. At $20 \mathrm{~min}, 80 \mathrm{~min}$, or $10 \mathrm{~h}$ post infection, infected cells were fixed and imaged using confocal microscopy. Imaris (Bitplane) was used to identify co-localization of RabGFP proteins with the bacteria containing vacuoles (Figure 3-3). By $10 \mathrm{~h}$ post infection, no E. coli could be identified, which is evidence of bacterial degradation. At early time points when $E$. coli was visible, less than $10 \%$ of $E$. coli vacuoles co-localized with Rab2b, Rab13, and Rab20. Similarly, Rab13 was not observed co-localizing with Y. pestis over that observed for $E$. coli at the time points studied. However, Y. pestis co-localized with Rab2b and Rab20 at a significantly higher frequency than $E$. coli ( $p ; \leq 0.01$ and $\leq 0.05$, respectively), indicating recruitment of both Rab proteins to the YCV by 80 min post infection. To determine if $Y$. pestis actively recruited Rab2b and Rab20 to the YCV, macrophages were infected with PFA-fixed Y. pestis. Similar to E. coli, no PFAfixed Y. pestis was observed at $10 \mathrm{~h}$ post infection. As with both $E$. coli and live Y. pestis, Rab13 did not co-localize with PFA fixed Y. pestis. Rab2b and Rab20 co-localized with PFA fixed Y. pestis to intermediate levels between $Y$. pestis and E. coli.

Repeated attempts to overexpress Rab22a consistently resulted in cell death. Therefore, I was unable to define the localization of Rab22a using this method. The lack of viable cells upon Rab22a overexpression may suggest Rab22a trafficking within RAW264.7 is important for cell viability. Rab22a mediates transfer of endosomes into recycling endosomes and it is possible that overexpression of Rab22a causes endosomes to recycled back to the plasma membrane and thus fails to deliver nutrients or signaling factors from the media to important intracellular locations. 


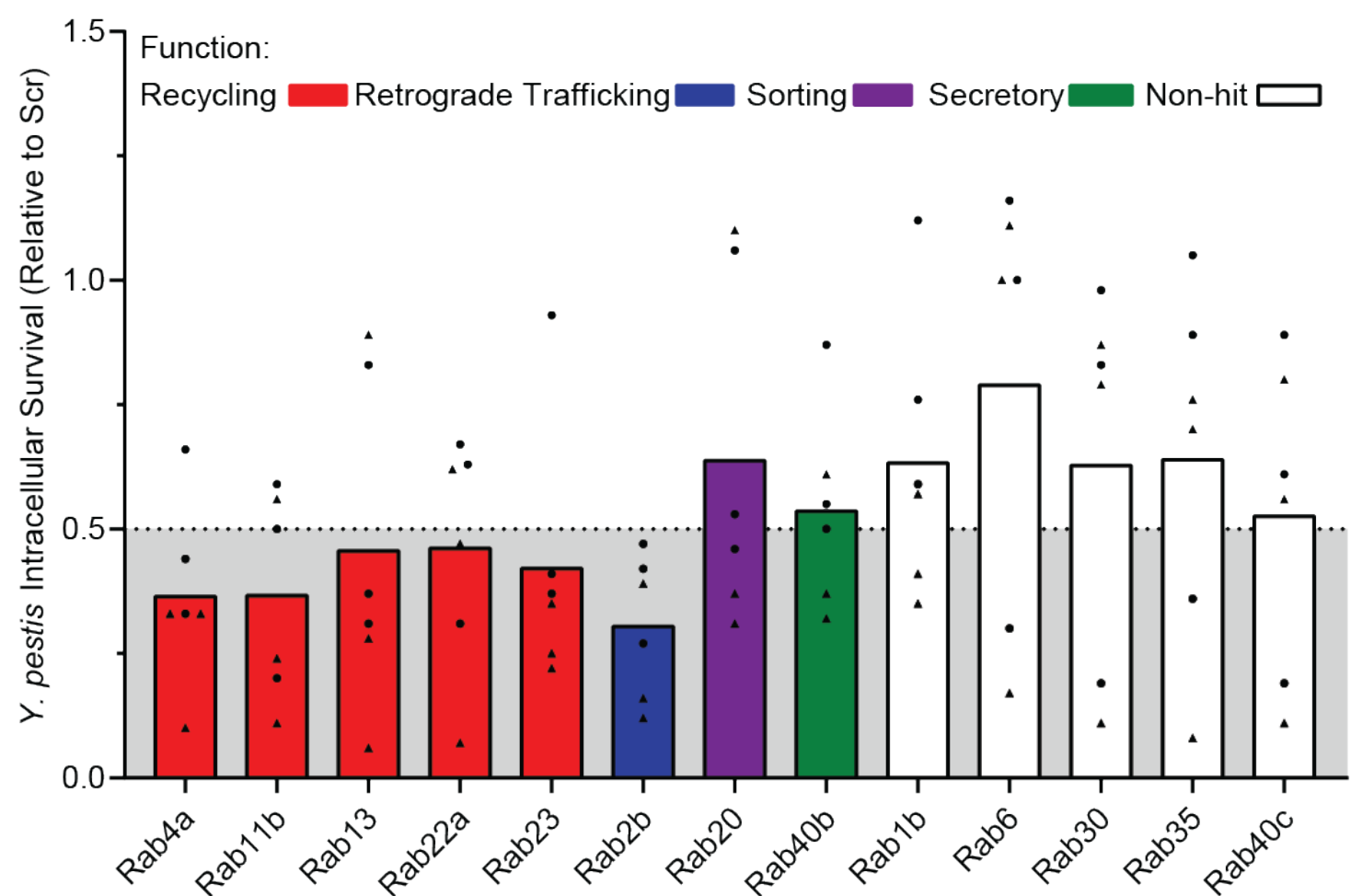

Figure-3-2: Rab proteins identified as essential for $Y$. pestis intracellular survival from deconvoluted validation.

RAW 264.7 macrophages were transfected with three siRNAs targeting 13 different Rab genes. Forty-eight hours after transfection, cells were infected with Y. pestis CO92 pCD1(-)LuXPtolC (•circles) or KIM D19 pCD1(+) LuXPtolc ( $\mathbf{\Delta}$ triangles) (MOI of 10) and intracellular bacterial numbers were determined by bioluminescence $(\mathrm{RLU})$ at $2 \mathrm{~h}$ or $10 \mathrm{~h}$ post-infection. Scrambled (Scr) controls were used as negative controls. Data is shown as the mean of percent of intracellular bioluminescence at $10 \mathrm{~h}$ post infection compared to $2 \mathrm{~h}$ post infection. Predicted trafficking pathways each identified Rab belongs to is indicated by color: red = recycling; blue = retrograde trafficking; purple =sorting; green = the secretory pathway; white $=$ did not meet cutoff criteria 


\section{DISCUSSION}

The role of three Rab GTPases in Y. pestis intracellular survival have been previously investigated to date $[118,119]$. Rab1b and Rab4a are recruited to the YCV early, and subsequently lost, while Rab11b co-localizes to the YCV throughout the course of infection $[118,119]$. Rab1b and Rab4a are required to subvert phagosomal acidification, whereas Rab11b is not. Instead Rab11b targeting appears to disrupt host cell recycling by sequestration of Rab11b to the YCV [119]. Moreover, we also showed that targeting and sequestration of Rab11b by $Y$. pestis impacts the transition of the YCV into a spacious vacuole, and Rab11b is required for autophagy, so that targeting of Rab11b may link $Y$. pestis to autophagy through the host cell recycling pathway during infection $[119,127,130]$. Recruitment/sequestration of Rab GTPases by Y. pestis may be mediated by bacterial effector proteins that modify GTP to GDP, or vice versa transitions, ability to occur. Alternatively, Rab GTPase insert into membranes can be altered via prenyl group modifications. Pathogenic bacteria commonly use these modifications to avoid degradation by macrophages [239], but whether such modifications occur in the context of $Y$. pestis infection have yet to be explored.

Here I have expanded on our understanding of the role of Rab proteins in Y. pestis pathogenesis by using a Rab specific RNAi screen to identify those Rab GTPases required for intracellular survival. All screens require an established criteria to identify hots that have the greatest impact on the observed phenotype. In this case, we used a cutoff of $>50 \%$ decrease in $Y$. pestis intracellular survival. It is possible that the Rab GTPases approaching a 50\% reduction of intracellular survival may also contribute to $Y$. pestis intracellular survival and YCV biogenesis could be considered as areas for future study. However, we focused on those Rab proteins that when knocked down, individually had the greatest impact on $Y$. pestis intracellular survival. Specifically, I demonstrated that six Rab proteins in addition to Rab1b, 4a, and $11 \mathrm{~b}$ are required for $Y$. pestis intracellular survival. Four of these Rab GTPases (Rab2, 20, 23, 40b) were also identified as potential hits in a previous whole genome pooled siRNA screen, but two (Rab13 and 22a) did not meet cutoff criteria in the original screen [119]. The use of a smaller format (39 versus $>17,000$ genes) and deconvolution for secondary validation may have allowed us to identify hits that were 
missed in the original screen. Three of these identified Rab targets (Rab13, 22a, and 23) have been linked to the host cell recycling pathway [253-255], which supports our previous study demonstrating that subversion of this pathway is an important step in avoiding killing by macrophages [119]. However, the other three Rab proteins have been previously suggested to be involved in the trafficking of the sorting endosome (Rab20), the secretory pathway (Rab40b), and retrograde trafficking (Rab2b) [256-258]. The importance of these Rab GTPases for intracellular survival suggest 1) pathways other than the recycling pathway are important for subversion of phagosomal maturation and formation of the YCV by Y. pestis, or 2) these Rab GTPases function within the recycling pathway, but their contributions have not been defined to date.

Upon phagocytosis, phagosomes enter the cells as sorting endosomes, which can traffic through either the phagosome maturation pathway or the recycling pathway, depending on cargo and subsequent association with downstream Rab GTPases. Rab20 has been shown to associate with the early endosome but not with late phagosomes [256, 259, 260]. In fact, retention of Rab20 on the early endosome prolongs retention of Rab5 (another Rab associated with endosome sorting [260]) and delays phagosome maturation and lysosomal fusion, indicating that Rab20 is a key regulator of phagosome maturation [256, 259]. During Y. pestis infection, I showed that Rab20 colocalized with the YCV within 20 min post-infection, and was retained over the course of infection. This was significantly different from E. coli, which did not appear to associate with Rab20 after 20 min post-infection. These data indicate that $Y$. pestis artificially retains Rab20 to the YCV, resulting in stalling phagosomal maturation, and perhaps allowing for subsequent entry in the recycling pathway. Rab20 has only been reported previously in the context of Mycobacterium infection, and has been implicated as a mechanism of restricting M. tuberculosis growth [261]. Schnettger et al. have shown that $M$. tuberculosis can rupture phagosomes to acquire nutrients in the host cytosol [261]. To prevent access to the cytosol, the host cell maintains an intact vacuole membrane through a Rab20 dependent mechanism [261]. Therefore, similar to autophagy, Rab20 appears to be detrimental for bacteria that access the cytosol. Moreover, these data support that $Y$. pestis remains in an intact vacuole and does not need to directly access the cytosol to survive within macrophages. Early retention of Rab20 on the YCV suggests that it may be key for Y. pestis to avoid phagosome 
acidification and lysosomal fusion, which should be directly tested in the future. Moreover, the potential role of Rab20 in autophagosome formation should be explored.

A

Rab2b

B

Rab13

C

Rab20
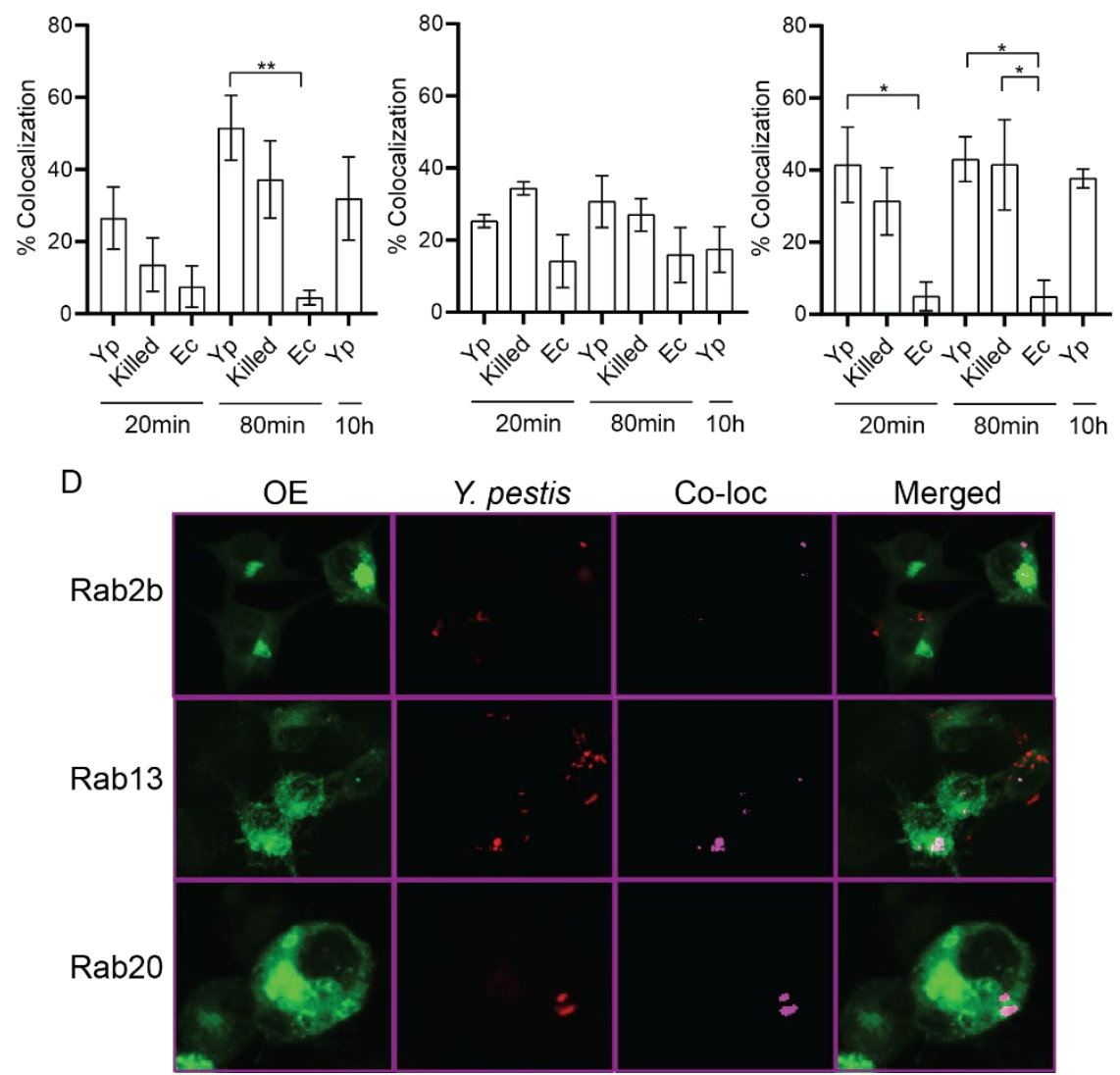

Figure 3-3: Rab2b, Rab13, and Rab20 co-localization in RAW264.7 macrophages.

RAW264.7 macrophages expressing indicated Rab-GFP proteins were infected with live Y. pestis CO92 pCD1(-) pGEN222::mCherry (Yp) (MOI 5), PFA-killed Y. pestis CO92 pCD1(-) pGEN222::mCherry (Killed) (MOI 5), or E. coli K-12 pGEN::mCherry (Eci) (MOI 20) (n=3) Frequency of co-localization of bacterium-containing vacuoles with transfected (A) Rab2b-EGFP (B) Rab13-EGFP, or (C) Rab20-EGFP Yp = Y. pestis; Killed = PFA fixed Y. pestis; Ec = E. coli. (D) Representative images of RAW264.7 macrophages transiently transfected with pEGFP-Rab (green) and co-infected with Y. pestis CO92 pCD1(-) pGEN222::mCherry. One-way ANOVA with Tukey's posthoc test was performed, and the results are indicated as follows: ns, not significant; *, $P \leq 0.05 ;{ }^{* *}, P \leq 0.01$. 
An essential process for maintaining membrane balance is the return of membrane from the golgi to the endoplasmic reticulum (ER) mediated by Rab2b, termed retrograde trafficking [262] [258]. Rab2b has also been linked to transport of exosomes out of the cell, which may indicate that there is more to be understood regarding the functions of Rab2b [263]. My data shows that unlike Rab20 which was observed to co-localize with $\mathrm{YCVs}$ by 20 min post-infection, Rab2b was recruited to the YCVs containing both live and PFA-fixed Y. pestis, by 80 min post-infection and retained for the duration of the infection. The co-localization to $Y$. pestis was significantly greater than that observed for E. coli, which did not appear to co-localize with Rab2b at 80 min post infection. These data indicate that $Y$. pestis infection induces recruitment of Rab2b to the YCV, which may function as a way for $Y$. pestis to recruit nutrients/membrane to the $\mathrm{YCV}$ for survival and replication. The only bacterium reported to interact with Rab2b is Brucella abortus, which targets Rab2b via the effector protein RicA [243], and modulates the Brucella containing vacuole into an ER like compartment [264]. Interestingly, silencing of Rab2b leads to diminished intracellular growth of $B$. abortus [264], similar to what is observed for $Y$. pestis. Acquisition of Rab2b by the YCV suggests that it may be a way for $Y$. pestis to directly alter phagosomal maturation or acquire nutrients/membrane, which should be explored in the future.

Interestingly, similar levels of Rab20 and Rab2b were observed on vacuoles containing live Y. pestis and killed Y. pestis vacuoles. The lack of difference between the live and PFA-fixed samples is unusual, as we previously showed that Rab1b, $4 a$, and $11 \mathrm{~b}$ recruitment to the UCV was dependent on live bacteria. This suggests that some factor conserved through fixation of $Y$. pestis, potentially a surface exposed ligand not shared with E. coli such as Ail, Pla, or another moiety, may impact the vesicular trafficking events leading to Rab2b and Rab20 co-localization. Additionally, active protein production may not be required for Rab20 retention and Rab2b recruitment. This observation may instead indicate that the bacterial factors responsible, are generated before macrophage interactions. Finally, it may also suggest that the method of entry into the cell may contribute to downstream trafficking events, which will be discussed in greater detail in Chapter 5 .

The recycling pathway is commonly targeted by pathogens as a way to avoid phagolysosomal maturation and degradation [245, 265-267]. Previously I discussed how Rab20 
delivers endosomes into the recycling pathways. Similarly, Rab13 is involved in delivering membranes from the Golgi to the recycling pathway [253]. Rab13 was not observed to be localized to the YCV or E. coli. Although Rab13 does not appear to be directly associated with the YCV, the phenotype observed upon silencing of Rab13 using siRNA may be due to an impact on host cell recycling apart from direct localization. Previously we published that $Y$. pestis stalls the recycling pathway likely through sequestration of Rab11b, potentially via a bacterial effector protein that recruits and retains Rab11b on the YCV; as overexpression of Rab11b restores recycling. Silencing of Rab11b expression does not result in $Y$. pestis death, but rather in the inability of $Y$. pestis to replicate, as $Y$. pestis remains at $10 \mathrm{~h}$ post infection without being degraded or replicating [119]. An alternative possibility for why Rab13 is important for $Y$. pestis intracellular survival, but does not localize to the YCV may attributable to interaction with Rab11b vesicles upstream of $Y$. pestis interception, thus indirectly contributing to $Y$. pestis survival by supplying membrane to the Rab11b dependent pathway. To date, exploitation of Rab13 has not been demonstrated as a host factor required for establishment of pathogen containing vacuoles. However, L. pneumophilia encoded LepB, a Rab1 GAP, is speculated to function as a GAP for additional Rab proteins including Rab13, although this has not been shown to occur in vivo [268]. One caveat that warrants consideration is that Rab13 interactions may be transient and thus missed in this analysis. Additional time points, or the use of a constitutively active form of Rab13 may help to identify whether transient Rab13 localization occurs. To better understand how Rab13 contributes to Y. pestis intracellular survival, further work is needed to evaluate whether Rab13 is critical for avoiding degradation or if it contributes to formation of the spacious YCV and replication.

Rab22a delivers endosomes to the slow recycling pathway, known to be important for $Y$. pestis survival [269-271]. Moreover, knockdown of Rab22a inhibits the return of transferrin through the slow recycling pathway, which we have previously is inhibited by $Y$. pestis infection of macrophages [119, 254]. Therefore, I formed the hypothesis that Rab22a is recruited by Y. pestis to the YCV in order to inhibit phagosomal maturation and divert to the slow recycling pathway instead. This would be similar to how M. tuberculosis and A. phagocytilim recruit Rab22a to their vacuoles in order to avoid degradation [113, 266, 272]. Although I was unable to determine if 
Rab22a is localized to the YCV using an overexpression construct, I speculate that Rab22a localizes to the YCV early during infection, which could be tested using a Rab22a antibody to assay endogenous Rab22a localization to the YCV. Additionally, the activity of Rab22a independent of localization relative to the YCV should be explored, such as the impact of Rab22a knockdown on acidification, induction of autophagy, and formation of the spacious YCV.

In conclusion, I have shown eight Rab GTPases, two of which were not identified by the Connor et al. genome wide screen, are important for $Y$. pestis intracellular survival within macrophages [119]. Moreover, I have shown that Rab2b and Rab20 are recruited to the YCV early during infection, while Rab13 is not. Defining the role of these Rab GTPases in avoidance of phagosome acidification and YCV maturation, in addition to defining the role of the other Rab GTPase screen hits will be important for understand how Y. pestis survives, replicates, and escapes from macrophages. In so doing, a clearer picture of how Y. pestis causes disease in the mammalian host will become evident. 


\section{CHAPTER 4}

\section{REDUNDANT AND COOPERATIVE ROLES FOR YERSINIA PESTIS YOP EFFECTORS IN THE INHIBITION OF HUMAN NEUTROPHIL EXOCYTIC RESPONSES REVEALED BY GAIN-OF-FUNCTION APPROACH ${ }^{1}$}

\footnotetext{
${ }^{1}$ Pulsifer AR, Vashishta A, Reeves SA, Wolfe JK, Palace SG, Proulx MK, Goguen J, Bodduluri SR, Haribabu B, Uriarte SM, Lawrenz MB. 2020. Redundant and cooperative roles for Yersinia pestis Yop effectors in the inhibition of human neutrophil exocytic responses revealed by gain-offunction approach. Infect Immun 88:e00909-19. ${ }^{1}$ PubMed PMID: 31871100.
} 


\section{INTRODUCTION}

Plague is the human disease caused by infection with the bacterial pathogen Yersinia pestis [1]. Depending upon the route of inoculation, plague can manifest in three forms [1]. Primary bubonic, pneumonic, or septicemic plague arises when bacteria are inoculated into the skin, lungs, or bloodstream, respectively. Upon infection with Y. pestis, mean time to death without medical intervention can range from 3 days for primary pneumonic or septicemic plague to 7 days for bubonic plague. A hallmark of $Y$. pestis infection is the lack of inflammation during early stages of colonization. During pneumonic plague in mice, a minimal inflammatory response is observed for the first 24 to $36 \mathrm{~h}$ of infection [37, 221, 273, 274]. Beginning at $\sim 48 \mathrm{~h}$ post-infection, the inflammatory response to $Y$. pestis changes, resulting in a significant increase in inflammatory mediators, including monocyte chemoattractant protein 1 (MCP-1), tumor necrosis factor alpha (TNF- $\alpha$ ), interleukin 12p70 (IL-12p70), gamma interferon (IFN- - ), and IL-6 [37, 221, 273, 274]. This coincides with an influx of immune cells, especially neutrophils, into the lungs, resulting in a rapid pneumonia $[37,221,273,274]$. Similarly, inflammation is delayed in bubonic plague and does not occur until after $Y$. pestis has begun to proliferate in the draining lymph node and disseminate [3, $4,36]$. The ability of $Y$. pestis to actively inhibit innate immune responses is a key virulence mechanism for $Y$. pestis [36, 37, 147, 273-275]. Normally, neutrophils are recruited in response to a variety of stimuli derived from damaged or activated host cells via damage-associated molecular patterns (DAMPs), cytokines, chemokines, or complement products [152, 231, 276]. Microbial components, such as lipopolysaccharide, peptidoglycan, or $\mathrm{N}$-formylmethionine-leucylphenylalanine peptides (fMLF), known as pathogen-associated molecular patterns (PAMPs), can also stimulate the recruitment of neutrophils [149]. Upon stimulation, neutrophils traverse the vasculature to reach the site of infection. Upon arrival at the site of infection, neutrophil antimicrobial responses are multifactorial and are comprised of phagocytosis, induction of the respiratory burst, degranulation, and release of neutrophil extracellular traps (NETs) [149]. Combined efforts from each of these responses make neutrophils very adept at killing microorganisms. Phagocytosis is important for clearing many bacterial infections, although some pathogens have acquired virulence 
factors that inhibit uptake by neutrophils $[165,277]$. In such situations, neutrophils rely upon extracellular release of antimicrobial mechanisms to effectively clear the infection.

One mechanism utilized by neutrophils to combat extracellular pathogens is the release of antimicrobial cargo contained in preformed granules (a process referred to as degranulation or graded exocytosis) [153]. Degranulation occurs in a regulated manner to coordinate release or modification of cytokines, chemokines, and signaling ligands/receptors to facilitate neutrophil transmigration and chemotaxis, with release of antimicrobial components that can directly restrict pathogen growth. Neutrophils contain four different granule subtypes, and mobilization of each granule is tightly controlled and dependent on the intensity of stimulation to coordinate functional responses [278]. Neutrophil degranulation is hierarchical, with secretory vesicles being the first subtype to undergo exocytosis, followed by gelatinase granules. Degranulation of specific and azurophilic granules, both loaded with toxic antimicrobial cargo, is more limited and requires stronger stimulation to promote granule mobilization [149]. Tightly graded control of granule release ensures that contents are released at the correct location to diminish collateral damage to the host.

The ability of neutrophils to mediate inflammatory responses has become more appreciated [279]. Neutrophils release a variety of cytokines and chemokines, as well as other immune modulatory factors that contribute to the cellular communication network during inflammation $[38,280]$. One of the most potent modulators released by neutrophils is leukotriene $\mathrm{B}_{4}\left(\mathrm{LTB}_{4}\right)$. Not only is it important for recruitment of additional neutrophils to the site of infection [181, 190, 281], but LTB4 also enhances the antimicrobial responses of both neutrophils and macrophages, including phagocytosis, respiratory burst, degranulation, and the release of inflammatory cytokines [196, 198, 203, 205, 209]. Importantly, LTB 4 production is not dependent on transcriptional regulation [195], and is therefore produced more rapidly than other chemoattractants, such as IL-8. Due to the rapidity of LTB 4 production, it is pivotal in mounting a swift inflammatory response $[198,202,208,209,282]$. Moreover, release of $\mathrm{LTB}_{4}$ is independent of degranulation [194], suggesting that regulation of LTB $_{4}$ release also differs from degranulation.

Although neutrophils are extremely capable of restricting microbial colonization, Y. pestis encodes a variety of virulence factors to evade recognition and killing by neutrophils $[43,48,53$, 
166, 283]. The Ysc type 3 secretion system (T3SS) secretes seven Yersinia outer protein (Yop) effectors directly into host cells and is paramount for inhibition and evasion of neutrophil responses $[40,147,166,221,226,284-286]$. Moreover, several in vivo studies have demonstrated that neutrophils are the primary cell type that $Y$. pestis interacts with during early stages of infection [42, 43, 221]. Once injected into neutrophils, Y. pestis Yop effectors interact with specific host factors to disrupt multiple host signaling pathways. YpkA, YopE, YopH, and YopT disrupt the actin cytoskeleton via interactions with host Rac, Rho, and focal adhesion complex proteins $[76,77,81$ 83, 225, 226, 285-295]. YopH has also been shown to inhibit host cell calcium flux [81, 296], while YopJ inhibits mitogen-activated protein kinase (MAPK) and nuclear factor kappa B (NF-kB) cascades [86, 224, 229, 284, 296]. Together, these Yop effectors have been shown to effectively inhibit neutrophil phagocytosis, respiratory burst, and cytokine/chemokine release $[86,166,224$, 284]. Importantly, the Yop translocon pore and effects of Yop effectors on host proteins can trigger inflammasome activation, which should lead to inflammatory responses [91, 297-299]. However, YopM and YopK function to inhibit inflammasome activation and subsequent inflammatory responses [91, 93, 97, 297, 299-302]. Together, the Yop effectors allow Y. pestis to actively inhibit the inflammatory response.

Recently it was shown that Yersinia pseudotuberculosis inhibits neutrophil degranulation in a T3SS-dependent manner, which was dependent on the actions of YopE and YopH [233]. Here, we show T3SS-dependent inhibition of neutrophil degranulation by $Y$. pestis, as well as roles of both YopE and YopH in inhibition. However, using a gain-of-function approach with a library of $Y$. pestis strains only expressing one Yop effector, we were able to identify additional Yop effectors contributing to inhibition of degranulation that have not been previously observed. Moreover, we show for the first time that $Y$. pestis actively inhibits production of $\mathrm{LTB}_{4}$ by human neutrophils, and we identify the Yop effectors contributing to this inhibition. 


\section{RESULTS}

$\underline{Y}$. pestis inhibits neutrophil degranulation in a T3SS-dependent manner.

Degranulation is a highly regulated but quick response that generally occurs within minutes after encountering a stimulus. Multiple studies have provided an understanding of the contents of the different granules that are released during degranulation (e.g., albumin is released during degranulation of secretory vesicles; gelatinase is released during degranulation of gelatinase granules), and the increased expression of receptors displayed on the neutrophil cell surface upon granule fusion with the plasma membrane (e.g., CD66b is displayed after degranulation of specific granules; CD63 is displayed after degranulation of azurophilic granules) (reviewed by Cowland and Borregaard [167]). Importantly, using these markers, degranulation of each granule subtype in response to different stimuli can be reliably monitored. Recently, it has been shown that $Y$. pseudotuberculosis inhibits degranulation by human neutrophils [233]. To determine whether $Y$. pestis similarly inhibits degranulation, human neutrophils were infected with Y. pestis CO92 or with a strain lacking the pCD1 plasmid encoding the Ysc T3SS [Y. pestis CO92 T3(-)]. At a multiplicity of infection (MOI) of 10 or 100, minimal, if any, release of the four granule subtypes was observed in response to Y. pestis CO92 (Figure 3-1). Similarly, at an MOI of 10, infection with Y. pestis CO92 $\mathrm{T}^{(-)}$did not result in degranulation. However, at an $\mathrm{MOI}$ of $100, \mathrm{Y}$. pestis $\mathrm{CO} 92 \mathrm{~T}^{(-)}$caused significant release of all four granule subtypes compared to infection with Y. pestis CO92 (Figure. 3-1 and Figure 3-2A and B). Surprisingly, using an $\mathrm{MOI}$ 10-fold higher, granules are not released in response to infection with Y. pestis $\mathrm{CO} 2 \mathrm{~T}^{(-)}$, indicating the T3SS is a potent inhibitor of granule release. Infection with $Y$. pestis KIM derivative with and without the $\mathrm{pCD} 1$ plasmid recapitulated the phenotypes observed for $Y$. pestis CO92 and CO92 $\mathrm{T}^{(-)}$, respectively. Together, these data, and data from a T3 effector-less mutant that produced the same phenotype as T3- (data not shown), indicate that degranulation is inhibited by $Y$. pestis in a T3SS-dependent manner. 
Cooperative inhibition of neutrophil degranulation by Yop effectors revealed through gain-offunction approach.

The Ysc T3SS delivers seven effector proteins into targeted host cells [303]. To determine if a single Yop effector is responsible for inhibiting neutrophil degranulation, human neutrophils were infected with a library of $Y$. pestis KIM1001 strains containing in-frame deletions of one yop gene (Table 2-1). While Y. pestis is able to inhibit release of all four granule subtypes (Figure 4-1), specific and azurophilic granules contain most of the antimicrobial components produced by neutrophils, and are typically released at the site of infection, where neutrophils would come into direct contact with $Y$. pestis. We therefore focused on the ability of $Y$. pestis Yop effectors to inhibit release of these two granule subtypes. Moreover, comparing the expression of degranulation markers after incubation with $Y$. pestis $\mathrm{T}^{(-)}$for 30 and 60 min indicated that degranulation peaked by 30 min post-infection (Figure $4-2 C$ and $D$ ). Therefore, degranulation was monitored at 30 min post-infection for subsequent experiments. As shown in Figure 3-3, infection with the Y. pestis KIM1001 $\mathrm{T3}^{(-)}$strain resulted in significant release of both specific and azurophilic granules compared to infection with mutants lacking any single yop gene. Each of the individual deletion mutants retained the ability to inhibit release of either granule, with surface expression of degranulation markers similar to that observed for Y. pestis KIM1001 (Figure 4-3). Similar results were observed with individual yop deletion mutants in the $Y$. pestis CO92 background (data not shown). These data suggest that more than one Yop effector protein is able to inhibit neutrophil granule release (i.e., functional redundancy in the system). 
A

Secretory Vesicle

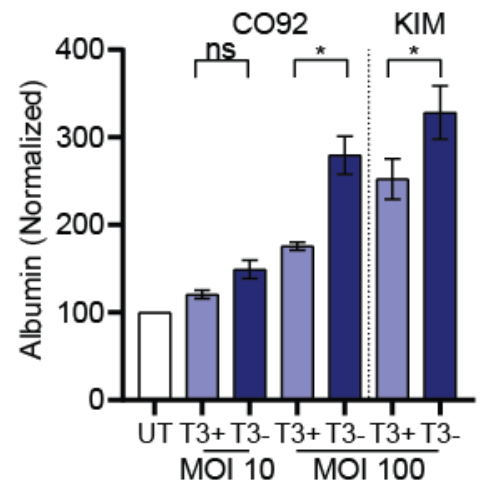

C

Specific Granule

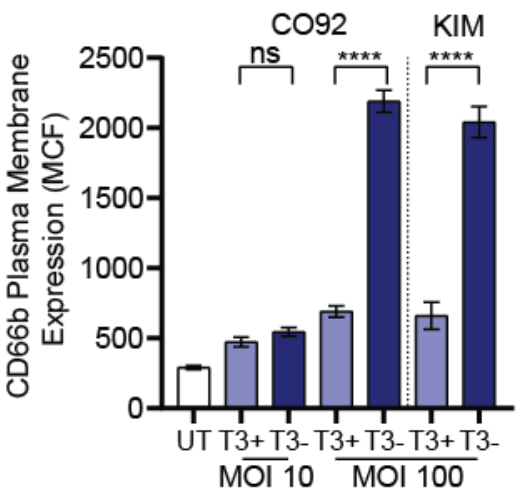

B Gelatinase Granule

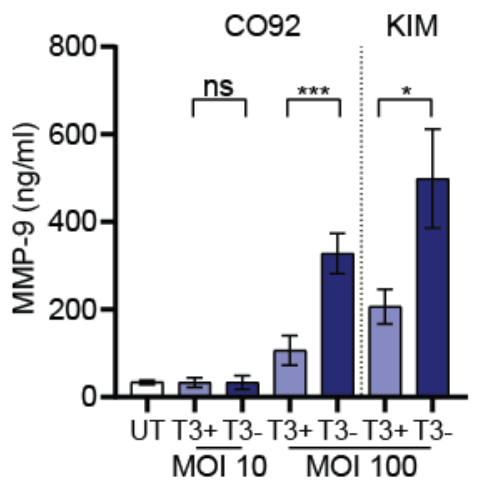

D Azurophilic Granule

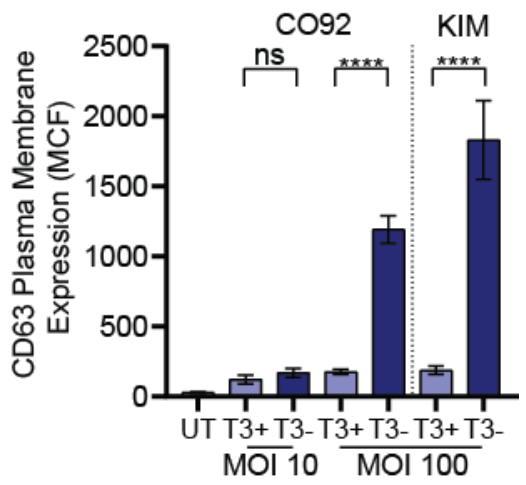

Figure-4-1: Y. pestis inhibits degranulation in a T3SS-dependent manner.

Human neutrophils $\left(4 \times 10^{6}\right)$ were infected with Y. pestis CO92 or Y. pestis KIM1001 with or without the pCD1 plasmid encoding the T3SS (T3+ or T3-, respectively) at indicated multiplicities of infection (MOIs) (10 or 100). Degranulation was measured after 30 min of infection for (A) secretory vesicles and (B) gelatinase, (C) specific, and (D) azurophilic granules. UT, untreated cells. Mean \pm standard error of the mean (SEM) from 5 biologically independent experiments. One-way analysis of variance (ANOVA) with Sidak's post hoc test; *, $\mathrm{P}<0.05 ;{ }^{* * *}, \mathrm{P}<0.001$; ${ }^{* * *}, \mathrm{P}<0.0001$. Representative flow cytometry histograms for specific and azurophilic granules are shown in Figure 4-2. 


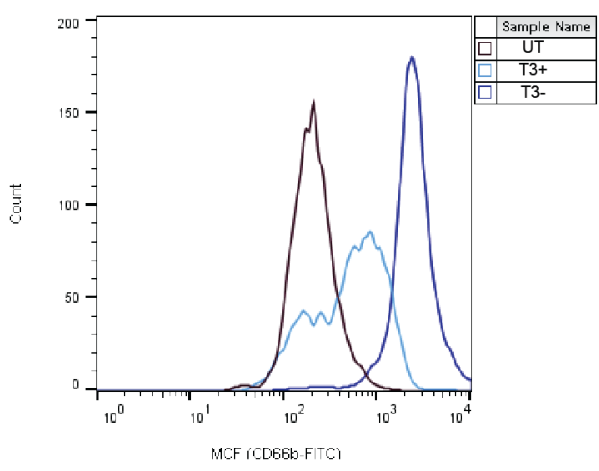

B Specific Granule

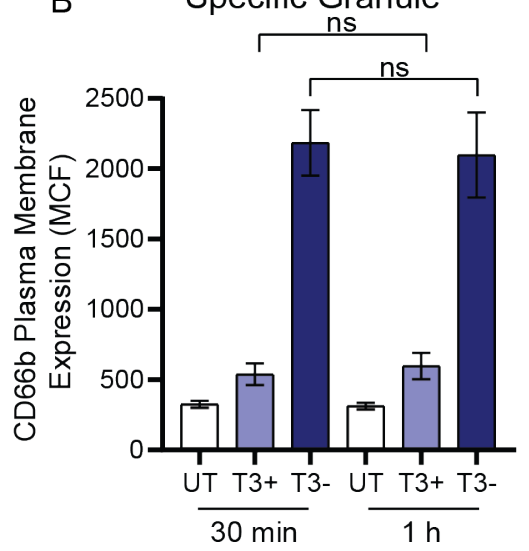

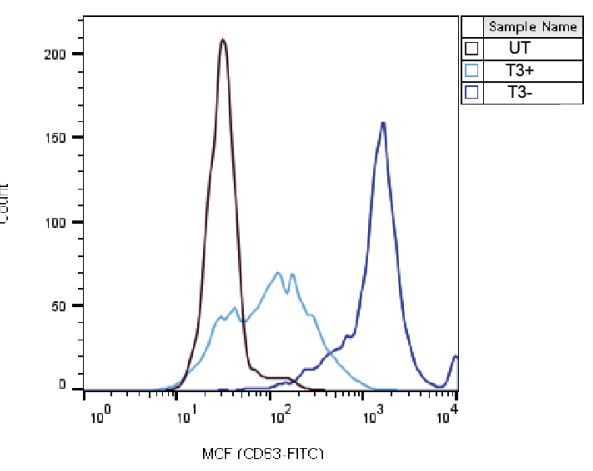

D Azurophilic Granule

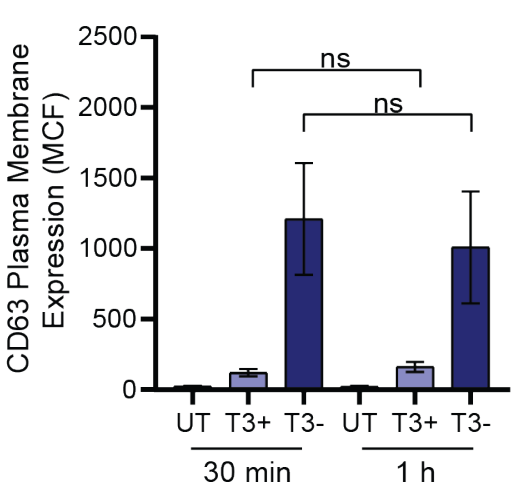

Figure-4-2: Degranulation of specific and azurophilic granules peaks by 30 min postinfection.

Human neutrophils $\left(4 \times 10^{6}\right)$ were infected with Y. pestis CO92 with or without the PCD1 plasmid encoding the T3SS (T3+ or T3-, respectively); MOI of 100. Degranulation of (A and B) specific and $(C$ and $D)$ azurophilic granules was measured $30 \mathrm{~min}$ and $1 \mathrm{~h}$ post-infection by flow cytometer. (A) and (C) Representative histogram for one experiment from (B) and (D) respectively. For (B) and (D) Mean \pm SEM from 4 biologically independent experiments. One-way ANOVA with Sidak's post-hoc; ns = not significant. UT = untreated cells. 
A

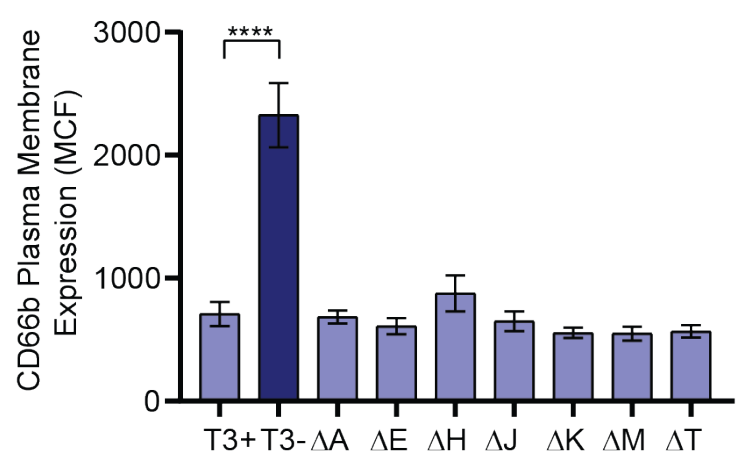

B

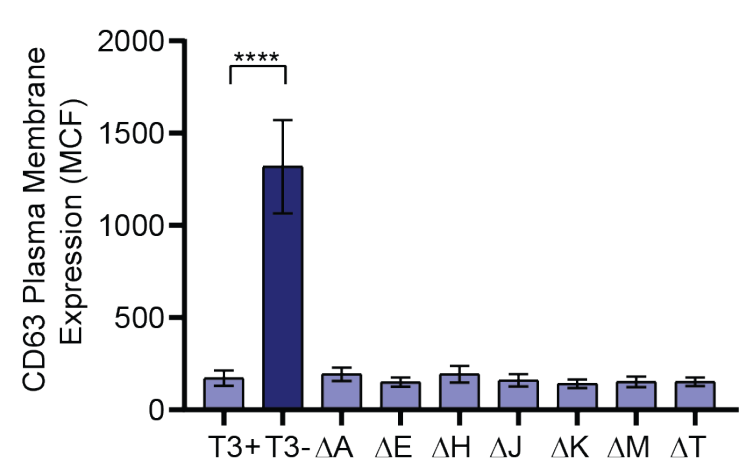

Figure-4-3: Deletion of individual Yop effector proteins does not alter neutrophil degranulation response to $Y$. pestis infection.

Human neutrophils $\left(4 \times 10^{6}\right)$ were infected with Y. pestis KIM1001 with or without the pCD1 plasmid encoding the T3SS (T3+ or T3-, respectively) or with strains lacking ypkA $(\Delta A)$, yopE $(\Delta E)$, yopH $(\Delta \mathrm{H})$, yopJ $(\Delta \mathrm{J})$, yopK $(\Delta \mathrm{K})$, yopM $(\Delta \mathrm{M})$, or yopT $(\Delta \mathrm{T})$; MOI=100. Degranulation was measured after 30 min of infection for (A) specific or (B) azurophilic granules. Mean \pm SEM from 5 biologically independent experiments. One-way ANOVA with Dunnett's post hoc test to T3-; ${ }^{* * * *}, \mathrm{P}<0.0001$. 
Recently, Palace et al. developed a library of $Y$. pestis strains that only express one Yop effector [229]. This library allows for the study of individual Yop effectors without the presence of the other six, which could confound data interpretation due to phenotypical masking by functionally redundant proteins. To determine whether individual Yop effectors inhibit degranulation, neutrophils were infected with strains from this library and monitored for exocytosis of specific and azurophilic granules (Figure 4-4). While strains expressing YopE, YopH, or YopT trended toward decreased specific granule exocytosis, none of the mutants demonstrated statistically significant decreases in exocytosis compared to the $T 3^{(-)}$strain (Figure 4-4A). Similar trends were observed for exocytosis of azurophilic granules for strains expressing YopE and $\mathrm{YopH}$, but surprisingly, the strain expressing only YopT caused increased release of azurophilic granules (Figure 4-4B). These data indicate that while there is functional redundancy for inhibiting degranulation by neutrophils, the effector proteins also work in a cooperative manner during Y. pestis infection to effectively inhibit exocytosis of specific and azurophilic granules.

YopE, YopH, YopJ, and YpkA act cooperatively to inhibit degranulation of specific and azurophilic granules.

To determine which Yop effectors act cooperatively to inhibit degranulation, a coinfection approach with two strains of $Y$. pestis expressing different individual Yop effectors was employed. Neutrophils were infected with a 1:1 mixture of two Y. pestis strains, each expressing different Yop proteins (final $\mathrm{MOI}$ is 100; $\mathrm{MOI}$ of 50 for each strain). Exocytosis of specific and azurophilic granules was compared to that of cells infected with $Y$. pestis $\mathrm{KIM} 1001 \mathrm{~T} 3^{(-)}$or a 1:1 mixture of $Y$. pestis KIM1001 and Y. pestis KIM1001 T3(-). As expected, coinfections with Y. pestis KIM1001 expressing all of the Yop proteins significantly decreased exocytosis of both specific and azurophilic granules compared to infection with only Y. pestis KIM1001 T3(-) (Figure 4-5 and 4-6). 
A

Specific Granule

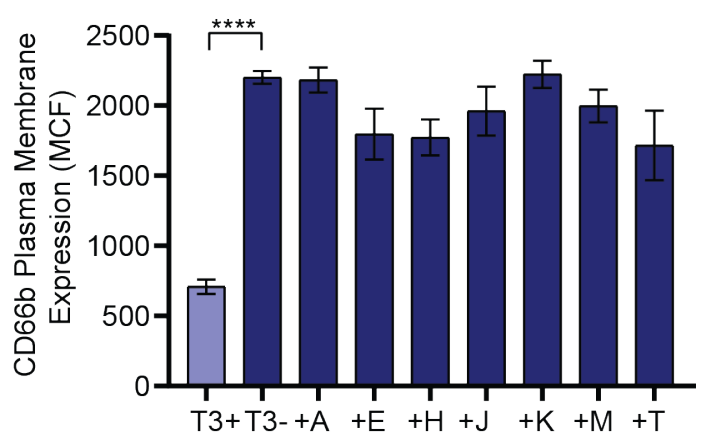

B

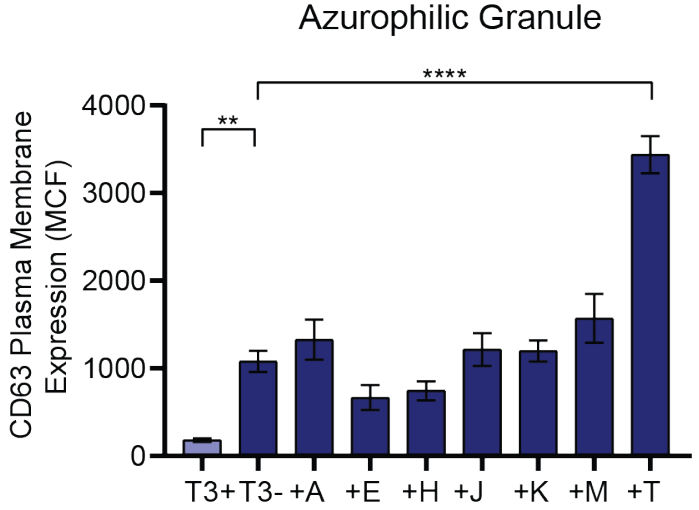

Figure-4-4: Individual Yop effector proteins are unable to completely inhibit degranulation. Human neutrophils $\left(4 \times 10^{6}\right)$ were infected with Y. pestis KIM1001 with or without the PCD1 plasmid encoding the T3SS (T3+ or T3-, respectively) or with strains expressing only ypkA $(+A)$, yopE $(+E)$, yopH $(+H)$, yopJ $(+J)$, yopK $(+K)$, yopM $(+M)$, or yopT $(+T)$; MOI=100. Degranulation was measured after 30 min of infection for (A) specific or (B) azurophilic granules. Mean SEM from 5 biologically independent experiments. One-way ANOVA with Dunnett's post hoc test to T3+: ${ }^{* *}, \mathrm{P}<0.01$; ${ }^{* * *}$, $\mathrm{P}<0.0001$. 
A

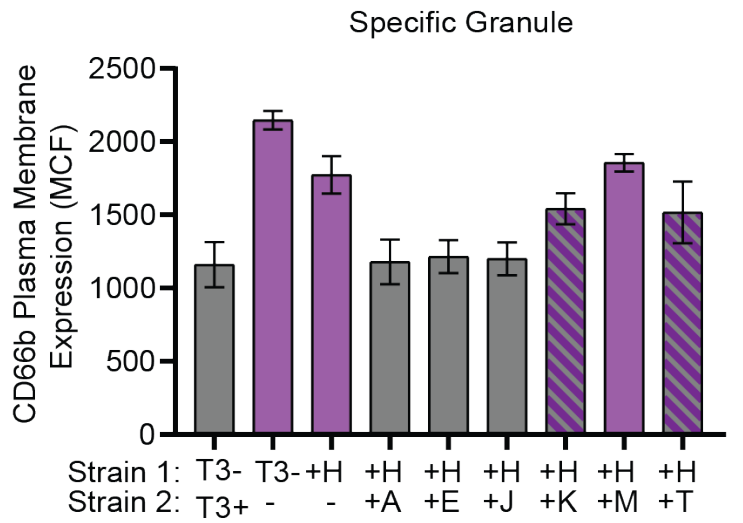

C

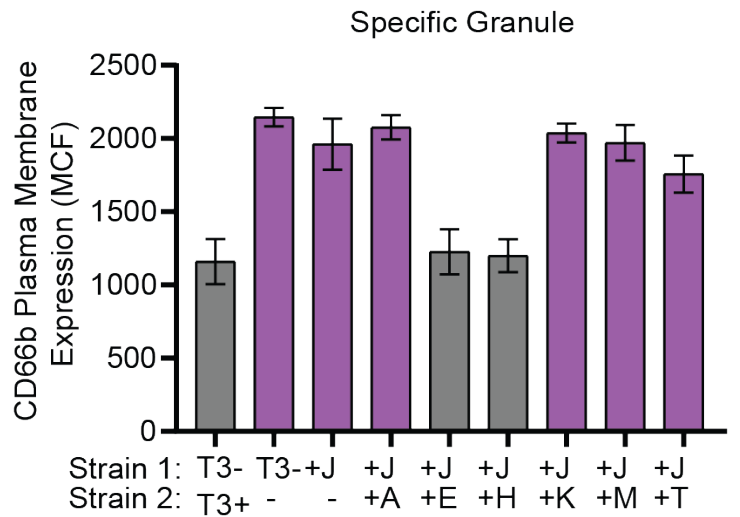

B

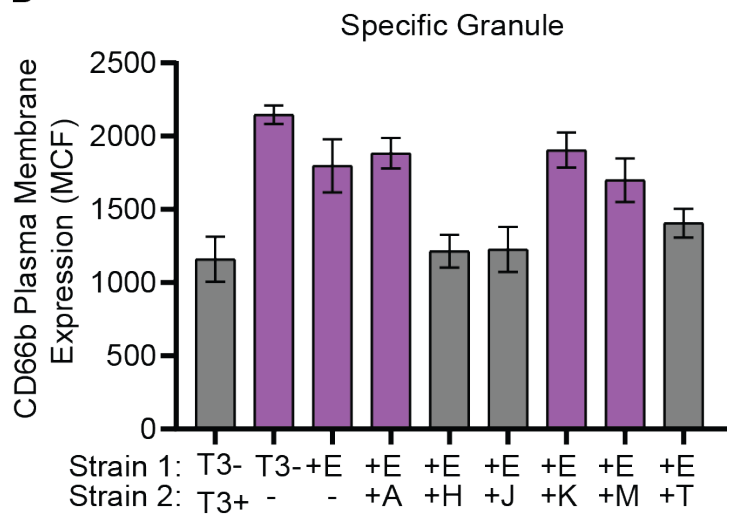

$\mathrm{D}$

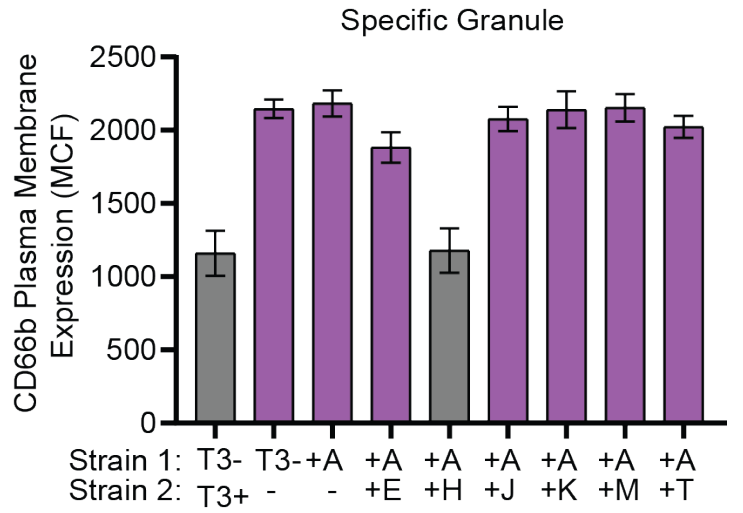

Figure 4-5: At least two Yop effector proteins are required to fully inhibit specific granule release.

Human neutrophils $\left(4 \times 10^{6}\right)$ were co-infected with Y. pestis KIM1001 with or without the pCD1 plasmid encoding the T3SS (T3+ or T3-, respectively) or with strains expressing only ypkA $(+\mathrm{A})$, yopE $(+\mathrm{E})$, yopH $(+\mathrm{H})$, yopJ $(+\mathrm{J})$, yopK $(+\mathrm{K})$, yopM $(+\mathrm{M})$, or yopT $(+\mathrm{T})$ mixed at a 1:1 ratio with strains expressing only $(\mathrm{A})$ yopH $(+\mathrm{H})$, (B) yopE (+E), (C) yopJ (+J), or (D) ypkA (+A); MOI of each strain was 50 for a combined $\mathrm{MOI}$ of 100 . Specific granule release was measured after $30 \mathrm{~min}$ of infection. Mean \pm SEM from 5 biologically independent experiments. One-way ANOVA with Dunnett's post hoc test. Gray bars are significantly different than T3- $(P<0.05)$; purple bars are significantly different from T3+/T3-. 
A
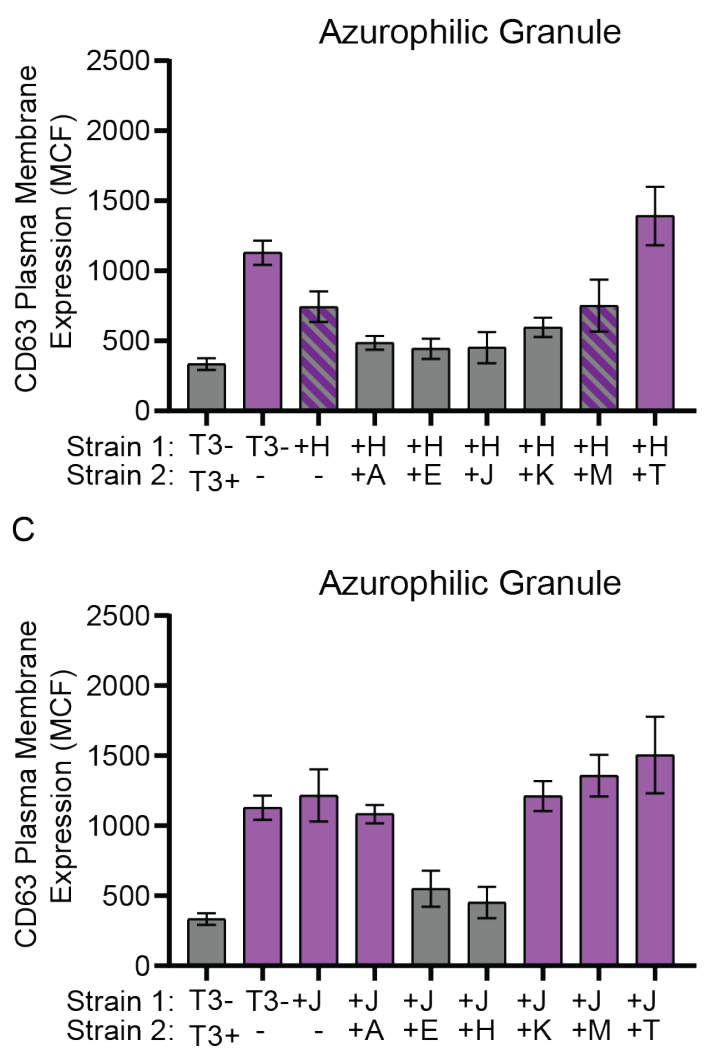

B
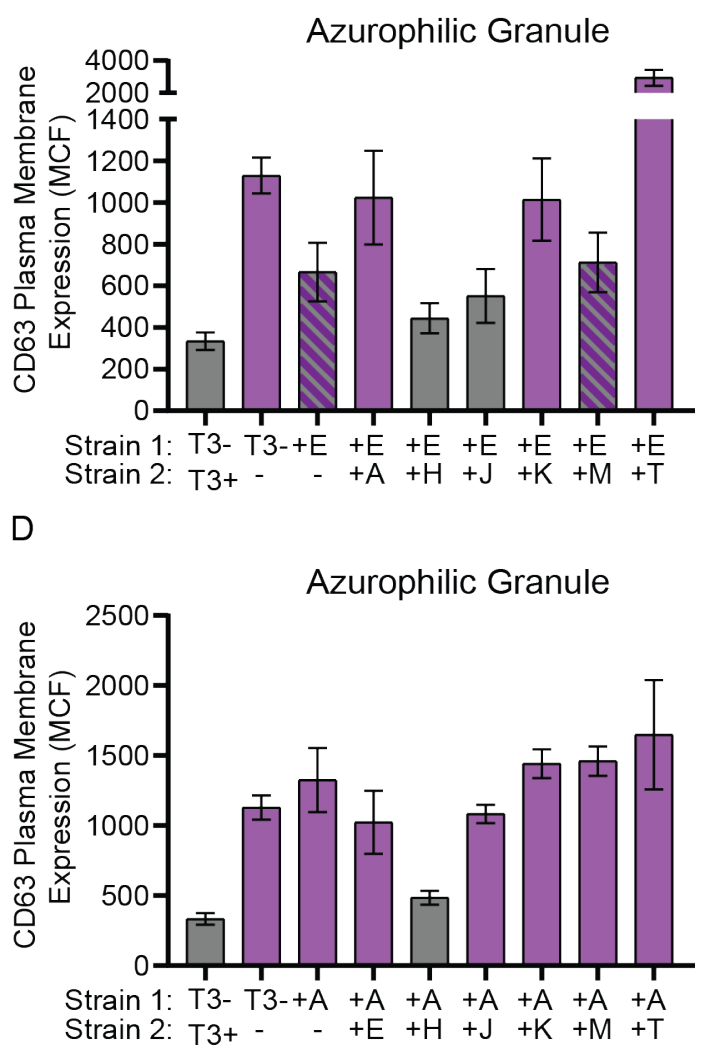

Figure 4-6: At least two Yop effector proteins are required to fully inhibit azurophilic granule release.

Human neutrophils $\left(4 \times 10^{6}\right)$ were co-infected with Y. pestis KIM1001 with or without the pCD1 plasmid encoding the T3SS (T3+ or T3-, respectively) or with strains expressing only ypkA $(+\mathrm{A})$, yopE $(+\mathrm{E})$, yopH $(+\mathrm{H})$, yopJ $(+\mathrm{J})$, yopK $(+\mathrm{K})$, yopM $(+\mathrm{M})$, or yopT $(+\mathrm{T})$ mixed at a $1: 1$ ratio with strains expressing only (A) yopH (+H), (B) yopE (+E), (C) yopJ (+J), or (D) ypkA (+A); $\mathrm{MOI}$ of each strain was 50 for a combined $\mathrm{MOI}$ of 100 . Azurophilic granule release was measured after $30 \mathrm{~min}$ of infection. Mean \pm SEM from 5 biologically independent experiments. One-way ANOVA with Dunnett's post hoc test. Gray bars are significantly different than T3- $(P<0.05)$; purple bars are significantly different than T3+/T3- $(P<0.05)$; hatched bars are not significantly different than T3- or T3+/T3-. 
Co-infection with two strains expressing only one Yop protein revealed that cooperative actions by four effectors were sufficient to inhibit degranulation of both specific and azurophilic granules. Coinfection with strains expressing YopH and YopE, YopH and YpkA, YopH and YopJ, or YopE and YopJ was sufficient to inhibit degranulation of both granules to levels similar to coinfection with Y. pestis KIM1001 and Y. pestis KIM1001 T3(-) (Figure 4-5 and 4-6). Coinfection with YopH and YopK appeared to also sufficiently inhibit release of azurophilic granules. For specific granules, coinfection with YopH and YopK, YopH and YopT, or YopE and YopT showed intermediate phenotypes. Coinfection with YopT could reverse the ability of YopH and YopE to partially inhibit degranulation of azurophilic granules (Figure 4-6A and B), reflecting the enhanced degranulation previously observed in single YopT infection (Figure 4-4B). However, coinfection with YopJ or YpkA appeared to inhibit the YopT enhanced degranulation phenotype (Figure 4-6C and D). Together, these data confirm previously reported roles for YopH and YopE in inhibition of degranulation [233], and also revealed previously hidden contributions of YpkA, YopJ, and YopK.

Y. pestis inhibits $\mathrm{LTB}_{4}$ response of human neutrophils.

$\mathrm{LTB}_{4}$ is a potent chemoattractant released by neutrophils independently of degranulation, and it contributes to early inflammation in response to infection [181, 194]. As inhibition of inflammation is a hallmark of $Y$. pestis infection, we next asked whether $Y$. pestis inhibits release of $\mathrm{LTB}_{4}$ by human neutrophils. Neutrophils were infected with Y. pestis KIM1001 or Y. pestis $\mathrm{KIM} 1001 \mathrm{~T}^{(-)}$, and the level of $\mathrm{LTB}_{4}$ released into the supernatant was compared to that released by untreated neutrophils (Figure 4-7A). Infection with Y. pestis KIM1001 did not result in significant release of $\mathrm{LTB}_{4}$ compared to untreated neutrophils. However, when neutrophils were infected with Y. pestis KIM1001 T3(-), a significant increase in $\mathrm{LTB}_{4}$ secretion was observed (Figure 4-7A; $P<0.01)$. To determine if these differences in $\mathrm{LTB}_{4}$ levels were sufficient to alter chemotaxis of naive neutrophils, conditioned supernatants from infected neutrophils were used in a chemotaxis assay and compared to supernatant from untreated neutrophils (Figure 4-7B). Naive neutrophils exposed to buffer or fMLF, a known chemoattractant, were used as controls. The numbers of naive neutrophils migrating toward the conditioned supernatant from untreated and Y. pestis KIM1001- 
infected neutrophils were not significantly different. However, in direct correlation with the elevated levels of

A

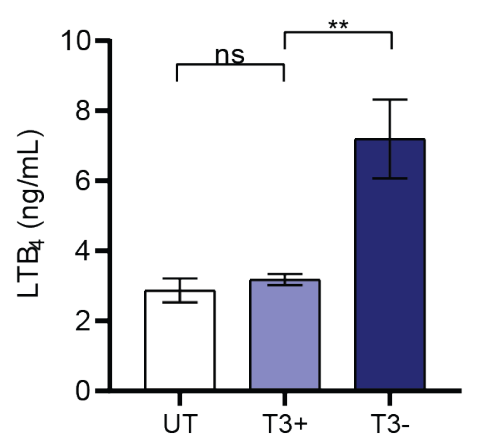

D

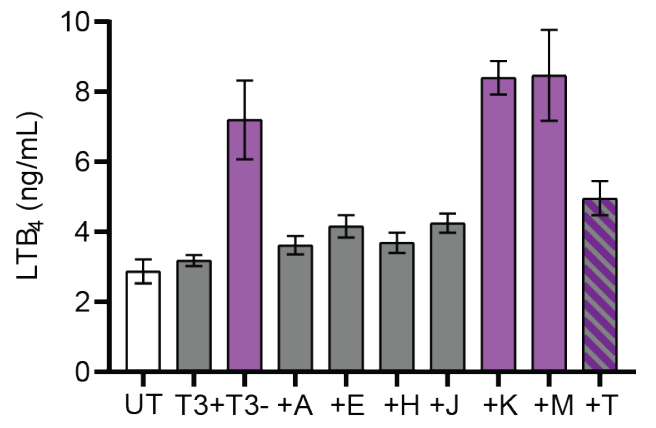

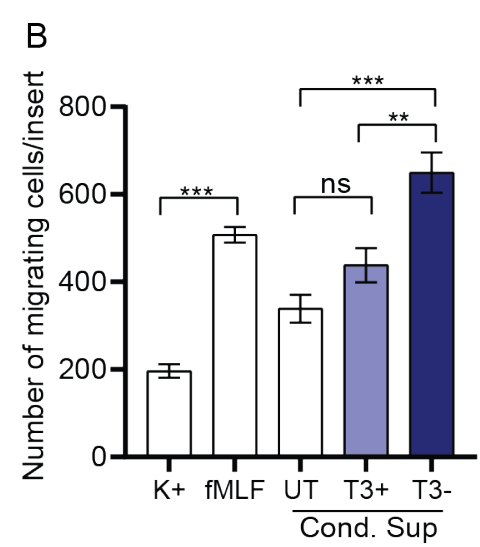

$\mathrm{E}$
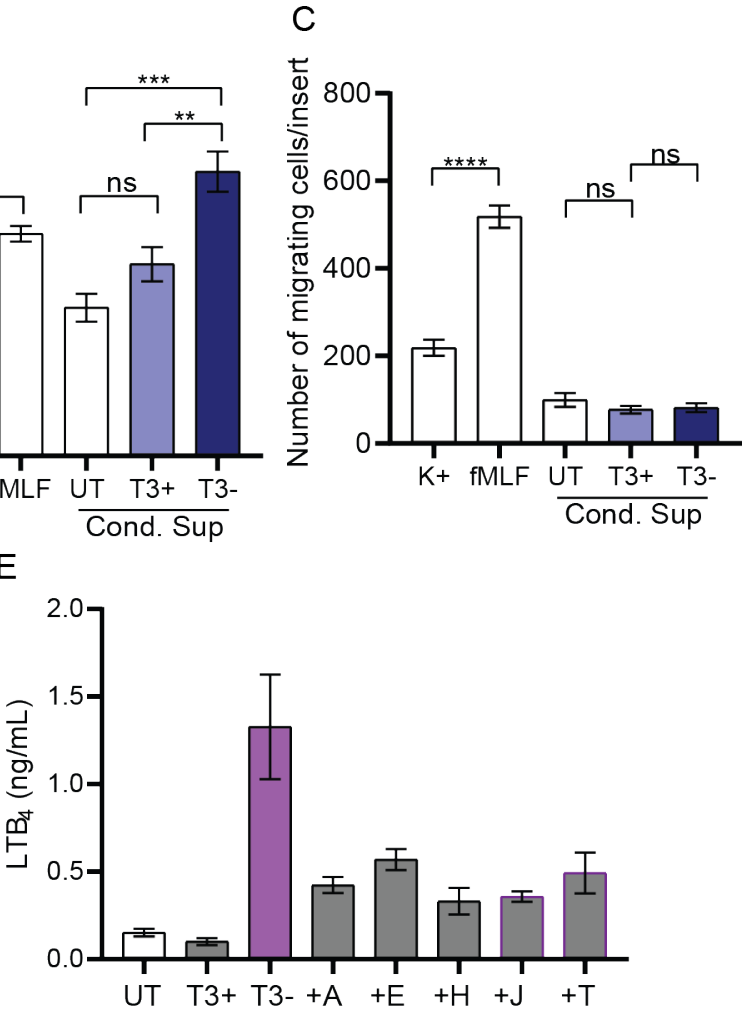

Figure 4-7: Y. pestis inhibits human neutrophil LTB4 response.

Human neutrophils $\left(4 \times 10^{6}\right)$ were infected with Y. pestis KIM1001 with or without the PCD1 plasmid encoding the T3SS (T3+ or T3-, respectively); $\mathrm{MOI}=100$. (A) Release of $\mathrm{LTB}_{4}$ was measured after 30 min of infection in supernatant. ( $B$ and $C$ ) Chemotaxis of naive neutrophils in response to conditioned supernatant (B) without or (C) with pretreatment of the BLT1 inhibitor LY293111. (D) $\mathrm{LTB}_{4}$ concentrations in the supernatant or $(\mathrm{E})$ cell lysates of neutrophils infected with strains expressing only ypkA $(+\mathrm{A})$, yopE $(+\mathrm{E})$, yopH $(+\mathrm{H})$, yopJ $(+\mathrm{J})$, yopK $(+\mathrm{K})$, yopM $(+\mathrm{M})$, or yopT $(+\mathrm{T})$. Mean \pm SEM from 5 biologically independent experiments. One-way ANOVA with Dunnett's post hoc test. ${ }^{* *}, P<0.01 ;{ }^{* * *}, P<0.001 ;{ }^{* * *}, P<0.0001$; ns, not significant. (D and $\left.E\right)$ Gray bars are significantly different than T3- $(P<0.05)$; purple bars are significantly different from T3+/T3$(P<0.05)$; hatched bars are not significantly different from T3- or T3+/T3-. 
$\mathrm{LTB}_{4}$ in the conditioned supernatant, significantly more neutrophils migrated toward the supernatant collected from cells infected with Y. pestis KIM1001 T3 $3^{(-)}$(Figure 4-7B; $P<0.01$ ). Pretreatment of naive neutrophils with an inhibitor that blocks signaling through the $\mathrm{LTB}_{4}$ highaffinity receptor BLT1 eliminated chemotaxis toward the conditioned supernatant but not toward fMLF (Figure 4-7C). These results indicate that the presence of $\mathrm{LTB}_{4}$ in the conditioned supernatant was promoting chemotaxis.

Next, we used the library of $Y$. pestis mutants expressing only one Yop effector to ask whether individual effector proteins are sufficient to inhibit $\mathrm{LTB}_{4}$ release. In contrast to the data observed for inhibition of specific and azurophilic granule release, four of the seven Yop effectors (YpkA, YopE, YopH, and YopJ) were able to inhibit LTB 4 release to levels similar to those of $Y$. pestis KIM1001 (Figure 4-7D). Moreover, infection with the strain only expressing YopT also substantially decreased the amount of $\mathrm{LTB}_{4}$ released from the neutrophils, although to a lesser degree than the other four effectors. Finally, to determine if $Y$. pestis infection inhibits synthesis or release of $L T B$, intracellular levels of $\mathrm{LTB}_{4}$ from infected neutrophils were measured. Similar to the results observed for conditioned supernatants, significantly lower amounts of intracellular LTB 4 were detected in cells infected with Y. pestis KIM1001 and mutants expressing YpkA, YopE, YopH, YopJ, and YopT (Figure 4-7E). Together, these data indicate that Y. pestis actively inhibits synthesis of $\mathrm{LTB}_{4}$ from human neutrophils in a T3SS-dependent manner, multiple Yop effectors are sufficient to inhibit $\mathrm{LTB}_{4}$ synthesis, and the inhibition of $\mathrm{LTB}_{4}$ release by infected neutrophils negatively impacts the chemotactic activity of naïve neutrophils to respond to the infection.

Disruption of the host cytoskeleton inhibits $\mathrm{LTB}_{4}$ release in response to $Y$. pestis infection.

Although different mechanisms are used by YpkA, YopE, YopH, and YopT, all four proteins have been shown to affect actin cytoskeletal rearrangement in host cells [225, 285, 304-306]. Because of this common effect, we hypothesized that $Y$. pestis disruption of the actin cytoskeleton could inhibit $\mathrm{LTB}_{4}$ release. If true, the release of $\mathrm{LTB}_{4}$ observed during infection with $Y$. pestis KIM1001 $\mathrm{T3}^{(-)}$could be blocked by artificially disrupting the actin cytoskeleton. To test this hypothesis, human neutrophils were incubated with latrunculin A, a chemical inhibitor of actin polymerization, prior to infection with $Y$. pestis, and $\mathrm{LTB}_{4}$ released into the supernatant was 
measured. As previously observed, significantly higher levels of LTB 4 were secreted by neutrophils treated with the vehicle and infected with Y. pestis $\mathrm{KIM}_{1001} \mathrm{~T}^{(-)}$than by vehicle-treated neutrophils infected with $Y$. pestis KIM1001 (Figure 4-8A; $P<0.01$ ). However, treatment with latrunculin $A$ resulted in loss of $\mathrm{LTB}_{4}$ release in response to the strain lacking the T3SS, supporting that actin cytoskeleton disruption by Yop effectors can inhibit the LTB 4 response in neutrophils.

\section{Disruption of MAPK signaling inhibits LTB 4 synthesis in response to $Y$. pestis infection.}

YopJ does not directly impact the host cell cytoskeleton, but it is a potent inhibitor of MAPK signaling $[71,87,307,308]$. Since MAPK signaling has been shown to control $\mathrm{LTB}_{4}$ synthesis in other models [71, 87, 307-312], we hypothesized that YopJ inhibition of LTB $_{4}$ synthesis is mediated by disruption of MAPK signaling. In vitro data indicate that YopJ can interact with multiple kinases in this pathway, including MAP3K (e.g., the TGF- $\beta$ activating kinase [TAK1]) and MAP2K (e.g., mitogen-activated kinase kinase 6 [MEK6]) [71, 302, 312-314]. Because TAK1 represents the earliest point in MAPK signaling targeted by YopJ, we tested whether treatment of neutrophils with a TAK1 chemical inhibitor was sufficient to inhibit $\mathrm{LTB}_{4}$ synthesis in response to Y. pestis KIM1001 $T 3^{(-)}$. As expected, when cells were exposed to the drug vehicle, we observed a significant increase in $\mathrm{LTB}_{4}$ release by neutrophils infected with $Y$. pestis $\mathrm{KIM}_{1001} \mathrm{~T}^{(-)}$compared to that by neutrophils infected with Y. pestis KIM1001 (Figure 4-8B; untreated, $P<0.001$ ). However, addition of the TAK1specific inhibitor (5Z)-7-oxozeaenol [(5Z)-7-oxo] inhibited this response by neutrophils, and no difference in $\mathrm{LTB}_{4}$ concentration was observed in the supernatants of neutrophils infected with $Y$. pestis KIM1001 or Y. pestis KIM1001 T3 ${ }^{(-)}$[Figure 4-8B; (5Z)-7-oxo]. TAK1 signaling is upstream of the MAPKs ERK and p38, but has not been shown to activate JNK in neutrophils [313]. To determine which MAPK was impacted by inhibition of TAK1 signaling, cell lysates from infected neutrophils were harvested, and the levels of phosphorylated p38 and ERK were measured by Western blotting. Compared to untreated neutrophils, we observed no difference in the phosphorylation of p38 during Y. pestis infection in the presence of the TAK1 inhibitor [Figure 4-8C and 4-9B; untreated versus (5Z)-7-oxo]. However, while phosphorylation of ERK was significantly increased in untreated cells during infection with Y. pestis KIM1001 T3(-), chemical inhibition of 
TAK1 signaling resulted in decreased ERK phosphorylation [Figure 4-8D; (5Z)-7-oxo], indicating that TAK1-

A

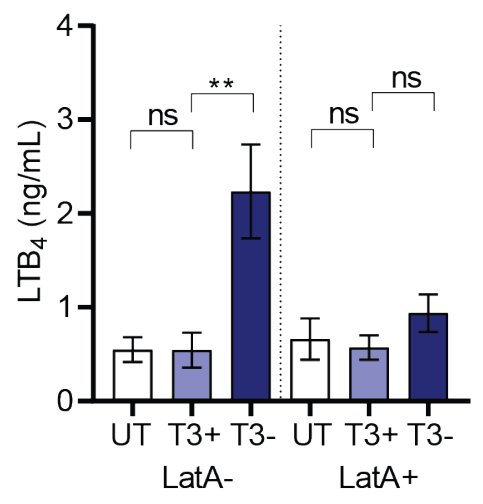

B

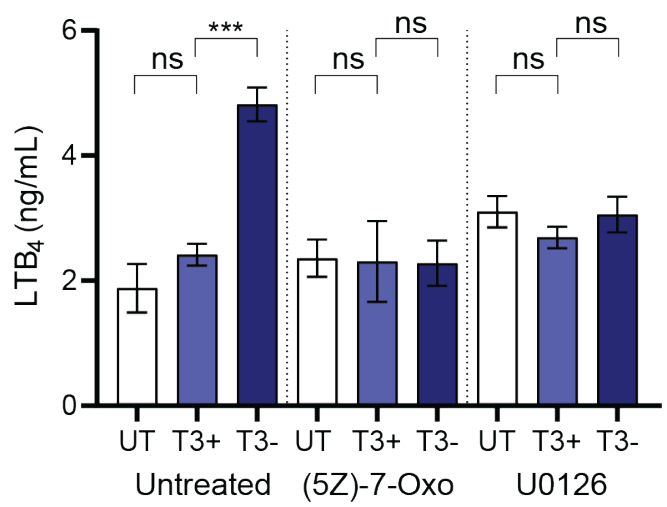

C

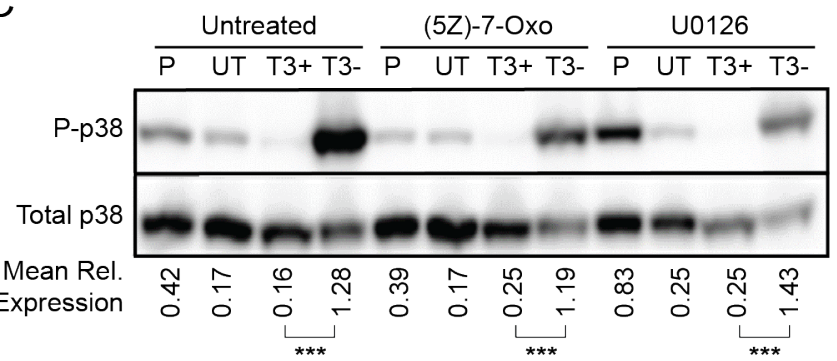

D

$\mathrm{E}$
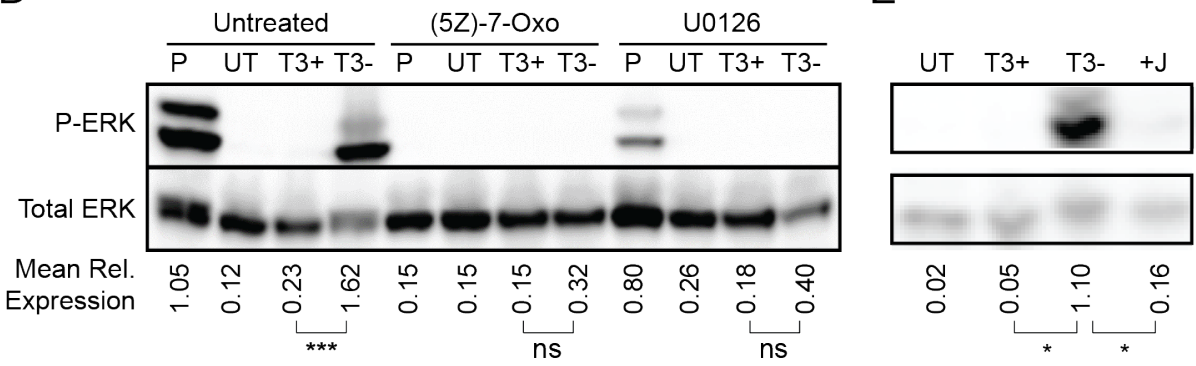

Figure 4-8: Inhibition of cytoskeletal rearrangement or MAPK signaling inhibits LTB 4 release.

Inhibition of cytoskeletal rearrangement or MAPK signaling inhibits LTB L $_{4}$ release. Human neutrophils $\left(4 \times 10^{6}\right.$ for LatA treatment or $8 \times 10^{6}$ for Western blots) were infected with $Y$. pestis KIM1001 with or without the PCD1 plasmid encoding the T3SS (T3+ or T3-, respectively); MOI=100. (A) Concentration of $\mathrm{LTB}_{4}$ in supernatant from infected neutrophils with indicated Y. pestis strains after pretreatment with vehicle control (LatA-) or latrunculin $A(L a t A+)$ prior to infection. (B) Concentration of $\mathrm{LTB}_{4}$ in culture supernatants after infection with indicated $Y$. pestis strains after pretreatment with vehicle control (untreated), the TAK1 inhibitor (5Z)-7-oxozeaenol [(5Z)-7-Oxo], 
or the ERK inhibitor U0126. (C) Phosphorylation of p38 and (D) ERK during infection with indicated strains after pretreatment with vehicle control (untreated), the TAK1 inhibitor (5Z)-7-oxozeaenol [(5Z)-7-Oxo], or the ERK inhibitor U0126. (E) Phosphorylation of ERK during infection with indicated Y. pestis strains. T3+, Y. pestis KIM1001; T3-, Y. pestis KIM1001 T3(-); +J, KIM1001 expressing only yopJ; UT, uninfected. (A and B) Mean \pm SEM from 5 biologically independent experiments. (C, D, and E) Mean relative expression calculated from 3 biologically independent Western blots. One-way ANOVA with Sidak's post hoc test. * $P<0.05 ;{ }^{* *}, P<0.01 ;{ }^{* *}, P<0.001$.

mediated activation of $\mathrm{LTB}_{4}$ synthesis during $Y$. pestis $\mathrm{KIM}_{1001} \mathrm{~T}^{(-)}$infection is through the ERK signaling pathway. To confirm that ERK signaling mediates LTB4 production in response to $Y$. pestis KIM1001 T3(-), neutrophils were treated with the ERK-specific inhibitor U0126 prior to Y. pestis infection. Similarly to treatment with the TAK1 inhibitor, blocking ERK signaling with U0126 inhibited the release of $\mathrm{LTB}_{4}$ in response to the Y. pestis KIM1001 T3 ${ }^{(-)}$strain (Figure 4-8B; U0126). Western blot analysis confirmed that U0126 specifically inhibited ERK phosphorylation and not p38 phosphorylation during $Y$. pestis $\mathrm{KIM} 1001 \mathrm{~T}^{(-)}$infection (Figure 4-8C and D and 4-9B; U0126). Importantly, infection with Y. pestis KIM1001 expressing only YopJ recapitulated the inhibition of ERK phosphorylation observed during infection with $Y$. pestis expressing all of the Yop effectors (Figure 4-8E and 4-9C), demonstrating that YopJ is sufficient to inhibit ERK signaling during $Y$. pestis infection. While inhibition of ERK signaling is sufficient to inhibit LTB4 release, the T3SS also inhibits phosphorylation of p38 in a non-TAK1 dependent manner (Figure 4-9A). Together, these data indicate that inhibition of ERK signaling in neutrophils by YopJ is sufficient to inhibit LTB4 synthesis during Y. pestis infection.

\section{DISCUSSION}

Through the T3SS and other virulence factors, Y. pestis is able to actively evade and inhibit the mammalian innate immune response, which allows the bacterium to colonize the host $[44,102$, 303]. Previous work has demonstrated targeting of resident and arriving neutrophils by $Y$. pestis for T3SS injection, which inhibits neutrophil antibacterial mechanisms that would otherwise result in bacterial killing $[40,42,43,55,166,221,222,224,315]$. Specifically, Y. pestis has been shown 
to inhibit phagocytosis [147, 166], reactive oxygen species production [147, 166, 284], and production of cytokines [224] by neutrophils. Our study further expands the understanding of how Y. pestis impairs the inflammatory response of host neutrophils by inhibition of neutrophil degranulation and $\mathrm{LTB}_{4}$ synthesis. Work in the closely related species $Y$. pseudotuberculosis demonstrated that the T3SS actively inhibits neutrophil degranulation via the contributions of YopE and YopH [233]. The Y. pseudotuberculosis study used adherent neutrophils, whereas we used neutrophils in suspension for several reasons. Adhering neutrophils causes partial activation and release of secretory vesicles, so that assaying secretory vesicle release would not be possible [316]. Furthermore, adherent neutrophils are also partially primed and more sensitive to further stimulus than neutrophils in suspension [212, 213, 316]. Moreover, interactions between bacteria and neutrophils are more random in suspension, and requiring higher MOls to observe the same phenotype using adherent neutrophils $[149,317]$. To understand the impact of $Y$. pestis infection on all four granule subtypes, neutrophils in suspension were used with an MOI higher than was used for the Y. pseudotuberculosis study [233].

Our data, and a recent report from Eichelberger et al. [318], demonstrate that $Y$. pestis also utilizes these two effector proteins to inhibit neutrophil degranulation. However, by using a gain-offunction technique, we were also able to identify the contributions of YopJ and YpkA to the inhibition of specific and azurophilic granule exocytosis. Moreover, and importantly, we were able to show that multiple Yop effectors must act cooperatively to inhibit degranulation. The likely reasons YopJ and YpkA contributions were missed previously are because (i) four different protein combinations can inhibit degranulation of both specific and azurophilic granules, and (ii) while four proteins are involved, the bacterium requires either YopH or YopE (i.e., YopJ and YpkA cannot inhibit without YopE or YopH). Therefore, using a conventional loss-of-function deletion approach, a yopE yopH double mutant will have a phenotype, while any other double mutation combination will not, leading to the erroneous conclusion that YopE and YopH are redundant and sufficient to inhibit degranulation. These data also suggest the potential for hidden contributions of Yop effectors to other previously described phenotypes identified via loss-of-function mutational approaches. For example, while YopJ has been linked to inhibition of IL-8 by neutrophils, a yopJ mutant does not 
release as much IL-8 as a T3SS-deficient mutant, suggesting cooperative actions by other Yop effectors [224]. Identification of other Yop effectors involved in inhibition could be performed using a similar gain-of-function approach to that described here.

A

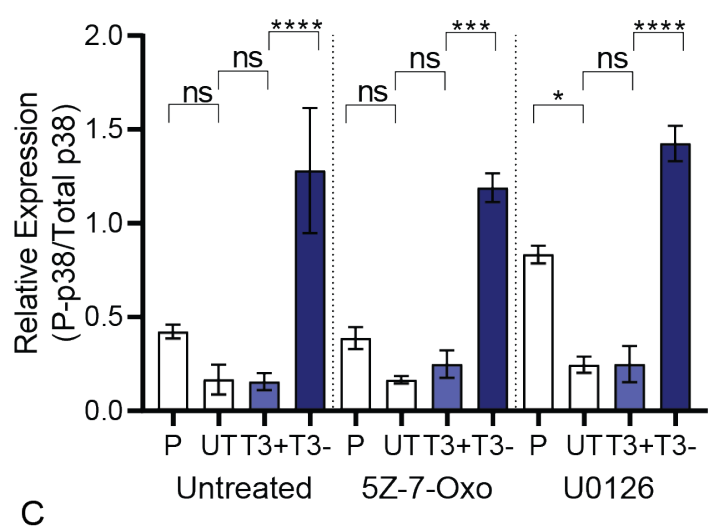

C

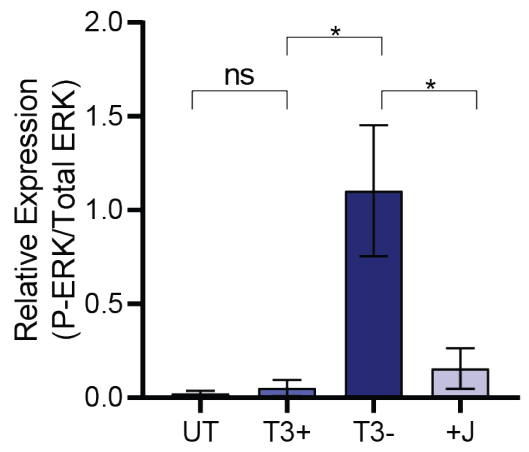

B

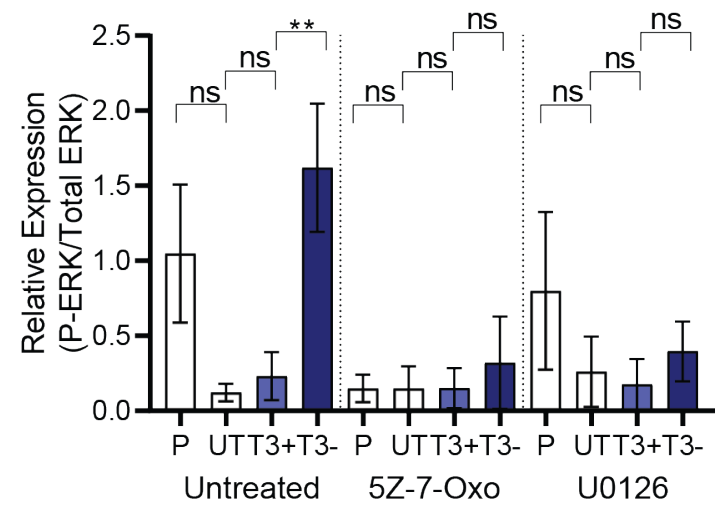

Figure 4-9: Quantification of p38 and ERK phosphorylation.

Human neutrophils $\left(8 \times 10^{6}\right)$ were infected with Y. pestis KIM1001 with or without the pCD1 plasmid encoding the T3SS (T3+ or T3-, respectively); $\mathrm{MOI}=100$. (A) Mean relative expression phosphorylated p38 or (B) phosphorylated ERK during infection with indicated strains after pretreatment with vehicle control (Untreated), the TAK1 inhibitor (5Z)-7-Oxozeaenol ((5Z)-7-Oxo), or the ERK inhibitor (U0126). (C) Mean relative expression of phosphorylated ERK during infection with indicated Y. pestis strains. Y. pestis KIM1001 $=$ T3+; KIM1001 T3(-) $=$ T3-; KIM1001 expressing only yopJ $=+\mathrm{J}$; Uninfected $=\mathrm{UT}$. Mean \pm SEM from 3 biologically independent experiments. Oneway ANOVA with Sidak's post-hoc; ns= not significant, ${ }^{*}, \mathrm{P}<0.05$. 
Previous work has shown that YopE and YpkA target Rac signaling [72, 77, 319], YopH targets the focal adhesion complex [285], and YopJ targets the MAPK signaling pathway $[71,86$, $87,295,308-310]$. All three of these host factors are key nodes in signaling pathways shown to be integral to regulating neutrophil granule release $[154,169]$. However, based on data from infections with single gain-of-function mutants, inhibition of one of these pathways by an individual Yop effector is not sufficient to inhibit degranulation. This suggests that individual signaling pathways may not be completely inhibited by the effector, or alternatively, that loss of signaling through one pathway can be compensated for in the neutrophil by signaling through the other pathways.

While the latter hypothesis may be supported by our observation that YopE and YpkA, which both target the same node/pathway, are not able to inhibit degranulation, our data do not rule out the former, as some degree of signaling through this node may still occur during coinfection with the YopE and YpkA strains. To overcome this hurdle, Y. pestis evolved to inhibit all three signaling pathways, with inhibition of at least two being sufficient to inhibit degranulation (an example of cellular process redundancy [320]). Importantly, the signaling pathways affected by these nodes are also important for other neutrophil antimicrobial mechanisms [153, 179, 216, 321]. Therefore, by targeting these host factors, $Y$. pestis is able to simultaneously inhibit multiple arms of the neutrophil response to subvert the functions of host neutrophils.

While the contributions of YopH, YopE, YpkA, and YopJ to inhibition of degranulation were conserved for specific and azurophilic granules, coinfections with YopH and YopK only appeared to inhibit the release of azurophilic granules. Based on the described function of YopK, which is thought to primarily regulate the translocation of other Yop effectors into the host cell to evade inflammasome recognition [93], we were surprised that YopK enhanced inhibition during coinfection with YopH. While YopK is thought to act as a gatekeeper, regulating the translocation of the other effectors from inside the cell [92], it has not been shown to regulate the transport of effectors through the T3SS of other bacteria during coinfection of a cell (i.e., transcomplementation). While it is possible that during coinfection YopK is trans-regulating the levels of YopH translocated by other bacteria, it is not clear how this would enhance inhibition of degranulation of azurophilic granules or why this would not also impact specific granules. 
Alternatively, it is possible that YopK has other yet-to-be defined functions in the host cells, beyond its role as a gatekeeper, that contribute to this phenotype, and future studies with YopK should be open to this possibility.

While YopE, YopH, YpkA, and YopT disrupt the host actin cytoskeleton, translocation of YopT by itself resulted in a phenotype that differed from the other three, enhanced azurophilic granule exocytosis (Figure -3-6B). Johnson et al. described Gem-interacting protein (GMIP), through RhoA GAP activity, controlling actin remodeling around the secretory Rab27a-JCF1 positive subpopulation of azurophilic granules to facilitate exocytosis [171]. Inhibition of actin polymerization by regulation of RhoA and ROCK activity releases the barrier that limits granule exocytosis [169]. Therefore, inactivation of RhoA by YopT is likely responsible for this phenotype. However, since this phenotype is specific for YopT, this suggests that YopT targeting of RhoA is spatially or temporally distinct from that of the other Yop effectors, that YopE and YpkA do not target RhoA during neutrophil infection, or that different mechanisms of RhoA inactivation by individual Yop effectors (e.g., protease cleavage versus GAP activity) may result in different degrees/rates of inactivation. Importantly, the action of the other Yop effectors inhibits this enhanced degranulation response in the context of wild-type (WT) Y. pestis infection to protect the bacterium from release of azurophilic granules.

Individually, YpkA, YopE, YopH, YopJ, and YopT all appear to be sufficient to inhibit LTB 4 synthesis. Synthesis of $\mathrm{LTB}_{4}$ requires activation and re-localization of the enzyme 5-lipooxygenase (5-LO) to a membrane such as the nucleus or endoplasmic reticulum or to recently described cytosolic structures called lipidosomes [190, 322, 323]. In this active state, 5-LO rapidly converts arachidonic acid to $\mathrm{LTA}_{4}$, which is followed by conversion to $\mathrm{LTB}_{4}$ by LTA 4 hydrolase $[189,190]$. The mechanisms leading to 5-LO translocation are not well defined. Moreover, whether the ratelimiting step for initiation of $\mathrm{LTB}_{4}$ synthesis is re-localization to membranes or bringing 5 -LO in proximity to 5-LO activating protein (FLAP) is still uncertain. However, 5-LO is known to associate with two actin-interacting proteins, growth factor receptor-bound protein 2 (Grb2) and coactosinlike protein (CLP) [324]. These interactions suggest that 5-LO translocation or interactions with FLAP require the actin cytoskeleton. This is further supported by our data, as four out of the five 
effectors that inhibit LTB 4 synthesis also disrupt the actin cytoskeleton. Moreover, treatment with the actin inhibitor latrunculin $A$ also inhibited $\mathrm{LTB}_{4}$ synthesis in response to $Y$. pestis $\mathrm{T}^{(-)}$. However, it is possible that individual effectors may inhibit the synthesis process at different steps, and identifying which steps are inhibited during $Y$. pestis infection is a future direction of our studies.

In addition to disruption of the host cytoskeleton, we have shown that $Y$. pestis is able to inhibit LTB 4 via YopJ disruption of ERK signaling. While YopJ inhibition of MAPK signaling has been extensively studied in the context of macrophages $[71,87]$, to our knowledge, this is the first time YopJ inhibition of MAPK phosphorylation has been confirmed in neutrophils. Specifically, our data demonstrates that both ERK and p38 phosphorylation are inhibited during Y. pestis infection of neutrophils in a T3SSdependent manner and that inhibition of TAK1-ERK signaling axis by YopJ is sufficient to inhibit $\mathrm{LTB}_{4}$ synthesis (Figure 3-8). In primary human neutrophils, TAK1 can differentially signal through ERK and p38, and phosphorylation of these MAPKs is dependent on the stimulus encountered by the neutrophils [313]. For example, stimulation of neutrophils with lipopolysaccharide (LPS) results in TAK1-mediated phosphorylation of both ERK and p38, while stimulation with granulocyte-macrophage colony-stimulating factor (GM-CSF) results in TAK1mediated regulation of the MEK/ERK axis [102]. Importantly, signaling via the TAK1-ERK pathway has also been shown to mediate $\mathrm{LTB}_{4}$ synthesis by neutrophils in response to other chemoattractant factors [102], supporting our findings that targeting ERK signaling by YopJ contributes to inhibition of $\mathrm{LTB}_{4}$ synthesis during Y. pestis infection. Importantly, MAPK signaling not only regulates $\mathrm{LTB}_{4}$ synthesis in neutrophils but also induction of the respiratory burst, production of cytokines, and degranulation [159, 162, 170, 188, 313, 325]. Therefore, targeting of MAPK signaling by YopJ and inhibition of TAK1-ERK-mediated signaling allows Y. pestis to disrupt many arms of the neutrophil response simultaneously.

In conclusion, $Y$. pestis is well adapted to surviving within the hostile host environment. Through this work, we found that neutrophils can only undergo granule exocytosis in response to Y. pestis infection when the T3SS is absent. In addition, the data presented here support previously described roles for YopE and YopH in inhibition of degranulation [233], while uncovering previously unidentified roles for YopJ and YpkA, which cooperatively work with YopE and YopH. Given these 
new data, we can also update the current model for inhibition of degranulation by $Y$. pestis to include the information that the bacterium needs to inhibit two of three signaling pathways to completely inhibit neutrophil degranulation. Moreover, Y. pestis also inhibits the synthesis of the potent chemoattractant $\mathrm{LTB}_{4}$. Without $\mathrm{LTB}_{4}$, neutrophil recruitment to the site of infection would be impaired. Moreover, as $\mathrm{LTB}_{4}$ also stimulates macrophages toward enhanced phagosomal degradation of microorganisms [198] and promotes dendritic cell activation of T-cell responses $[197,326,327]$, both of these important mechanisms to coordinate early antimicrobial responses by host innate immune cells are likely impaired during $Y$. pestis infection. Inhibition of neutrophil degranulation and $\mathrm{LTB}_{4}$ production likely contributes to $\mathrm{Y}$. pestis subverting the innate immune response and maintaining a non-inflammatory host environment early during infection. 
CHAPTER 5

ADDITIONAL OBSERVATIONS, DISCUSSION, AND FUTURE DIRECTIONS 


\section{RESEARCH SUMMARY}

The human immune system relies upon an interplay between cell types to produce a finely tuned response to microbial infection. The keystone cells mediating the initial response to infection are innate immune cells, namely macrophages and neutrophils $[165,181]$. Y. pestis has adapted to survive in the hostile mammalian host through subversion and inhibition of many innate immune cell responses [39, 41, 70, 166, 224]. Early inhibition of inflammatory signals maintains an environment permissive to $Y$. pestis replication $[36,40,146,274]$. Understanding how $Y$. pestis subverts and manipulates innate immune cell responses, sheds light on how the early noninflammatory response is maintained, allowing Y. pestis to survive and replicate within the host. My research efforts have contributed to further elucidating how Y. pestis uses Rab proteins within macrophages in order to establish a permissive intracellular environment and create a spacious YCV. I have identified eight Rab GTPases required for Y. pestis intracellular survival. Thus far five Rab proteins, Rab1b, 2b, 4a, 11b, and 20 have been found to be recruited to the YCV, while Rab13 is not (Figure 5-1). Manipulation of vesicular trafficking and Rab GTPases localization is likely how Y. pestis survives and replicates within macrophages, providing a niche that ultimately contributes to avoiding destruction by neutrophils prior to upregulating expression of the T3SS.

To better understand how neutrophils are also subverted by $Y$. pestis in order to survive and maintain an early non-inflammatory host environment as seen during pneumonic plague, I infected human neutrophils with $Y$. pestis and determined whether granule and $\mathrm{LTB}_{4}$ release was inhibited. I demonstrated that exocytosis of all four neutrophil granule/vesicle types are inhibited through a T3SS dependent manner. I also identified previously unknown contributions of YpkA and YopJ regarding inhibition of neutrophil degranulation. Working cooperatively, YopE with YopH or YopJ, or YopH with YpkA, YopE, YopJ, and potentially with YopK, inhibit the release of specific and azurophilic granules. Two of the three signaling pathways involved in neutrophil degranulation must be inhibited by the Yop effectors in order for azurophilic and specific granule exocytosis to be inhibited (Figure 5-2). As observed for degranulation, the Ysc T3SS is also able to inhibit release of $\mathrm{LTB}_{4}$ from neutrophils through the individual actions of YpkA, YopE, YopH, YopJ, and YopT. The 
five Yop effector proteins function by disrupting actin cytoskeletal rearrangement or TAK1/ERK signaling (Figure 5-3).

My efforts to better understand how $Y$. pestis survives within macrophages and subverts neutrophil antimicrobial responses, have guided me to three broad future questions that I think are important for better understanding what occurs during Y. pestis infection. The first question is: Mechanistically, how does Y. pestis modulate host vesicular trafficking to escape degradation and establish a replicative niche? The second question is: How does Y. pestis inhibit the release of neutrophil antimicrobial products mechanistically? Finally, the third question is: Are the neutrophil responses to $Y$. pestis infection the same between murine neutrophils and human neutrophils? I have made additional observations and proposed future directions for this work, which are detailed in the subsequent paragraphs. 


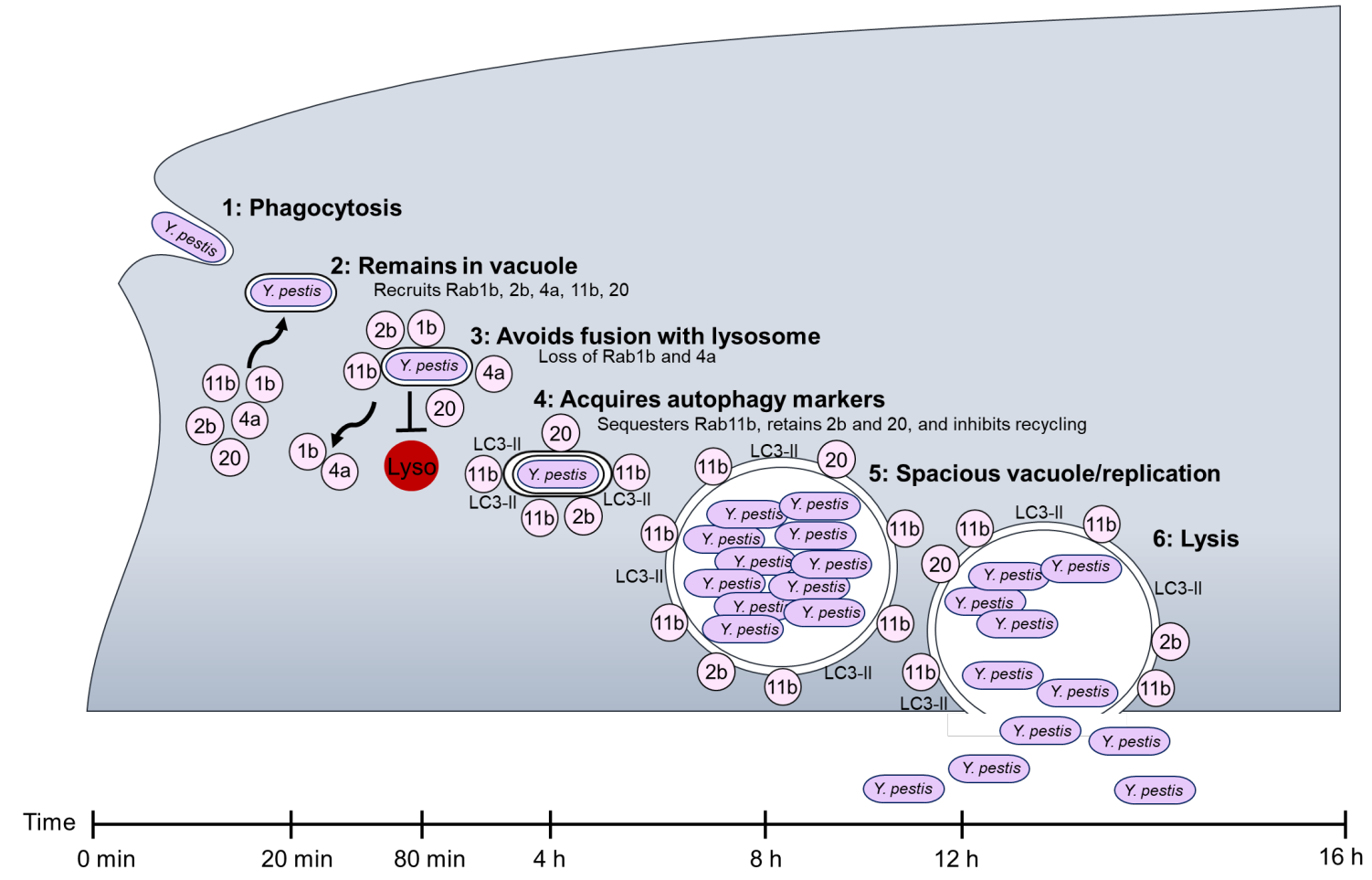

\section{Figure 5-1: Rab GTPases localize to the YCV.}

In order for $Y$. pestis to avoid degradation by macrophages, the YCV does not fuse to lysosomes and instead associates with markers of autophagy before replicating and lysining out of the cell. Many pathogens modulate Rab GTPases to alter pathogen containing vacuole trafficking events to avoid degradation. Y. pestis similarly uses Rab GTPases to avoid lysosomal degradation and to enter into a replicative niche. Y. pestis localizes with Rab1b, 2b, 4a, 11b and 20. While Rab1b and Rab4a no longer associate at late time points, Rab2b, $11 \mathrm{~b}$, and 20 remain to $10 \mathrm{~h}$ post infection. 


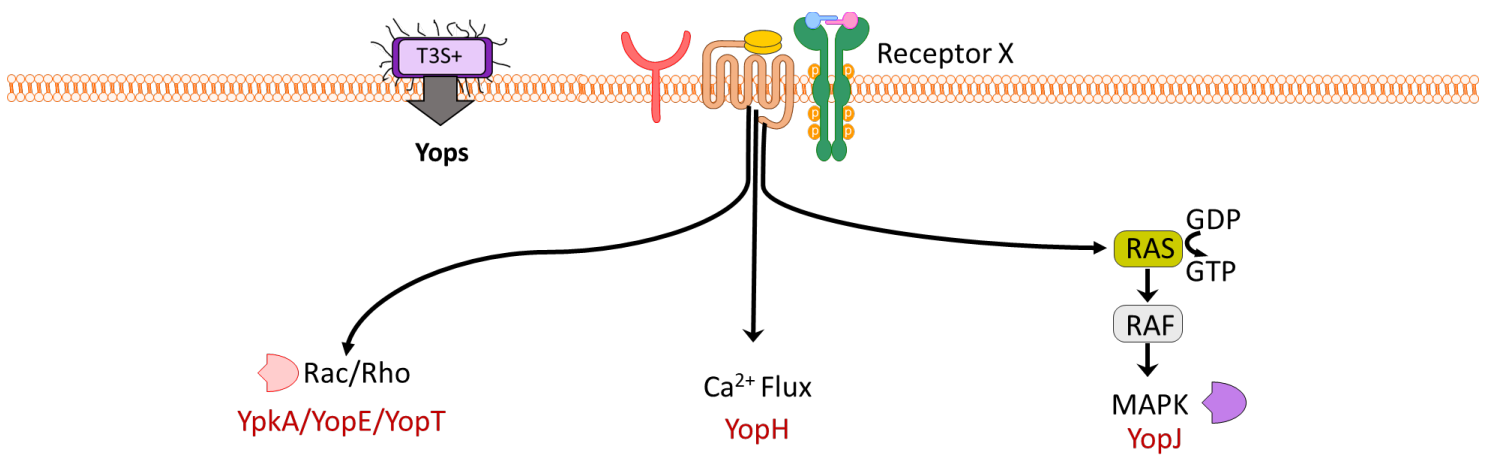

Granule exocytosis does not occur

Figure 5-2: Inhibition of neutrophil granule release by $Y$. pestis.

Granule release from neutrophils are regulated by three different signaling nodes, the MAPK cascade, Rac/Rho signaling, and the focal adhesion complex/Ca ${ }^{2+}$ flux. Yersinia pestis injects neutrophils with seven bacterial effector proteins that interact with host proteins to inhibit signaling from occurring. Inhibition of two of the three signaling nodes important for granule release by YpkA/YopE/YopT, YopH, and YopJ prohibits neutrophils from exocytosing granules in response to Y. pestis infection. During infection with WT Y. pestis, all three pathway nodes would be inhibited for maximal suppression of neutrophil granule release. 


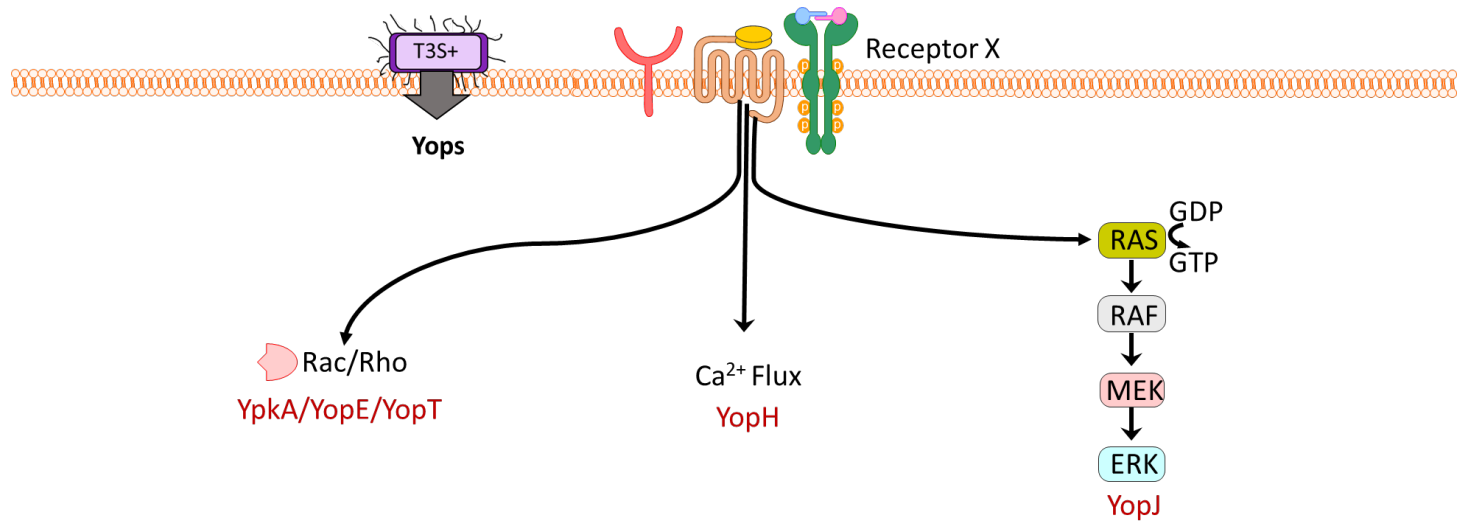

$\mathrm{LTB}_{4}$ synthesis does not occur

\section{Figure 5-3: Inhibition of LTB4 production by T3SS effector proteins.}

$\mathrm{LTB}_{4}$ production in neutrophils is regulated by multiple signaling pathways, the MAPK cascade, $\mathrm{Ca}^{2+}$ flux, and actin cytoskeleton rearrangement. Yersinia pestis injects seven bacterial effector proteins into neutrophil cytosol that interact with host proteins to inhibit signaling from occurring. Inhibition any one of the three signaling nodes by YpkA, YopE, YopT, YopH, or YopJ prohibits neutrophils from producing $\mathrm{LTB}_{4}$ in response to $Y$. pestis infection. During infection with WT $Y$. pestis, all three pathway nodes would be inhibited for maximal suppression of $\mathrm{LTB}_{4}$ production and release. 


\section{ADDITIONAL OBSERVATIONS AND QUESTIONS}

\section{$\underline{5.1 \text { How does } Y \text {. pestis survive and replicate within macrophages? }}$}

My research efforts have identified additional Rab proteins targeted by $Y$. pestis to establish a permissive intracellular environment not previously identified by the Connor et al. [119]. However, knowing that Rab GTPases are important for Y. pestis intracellular survival, and which Rabs colocalized to the YCV, only provides a partial story of what is happening within macrophages during infection. Further work is needed to fully understand how $Y$. pestis survives and replicates in macrophages. In the discussion of Chapter 3, I proposed several experiments to better understand the roles of Rab2b, 13, and 20 in Y. pestis intracellular survival. The following sections will instead focus on testing two potential hypotheses that may be used by $Y$. pestis to generate the YCV.

\subsubsection{Hypothesis 1: Y. pestis encodes effector proteins to modulate Rab protein trafficking.}

Eight macrophage Rab proteins were identified as important for $Y$. pestis intracellular survival. Other pathogens, such as $L$. pneumophila or S. typhimirium utilize bacterial encoded effector proteins that directly targets and modulates Rab protein activity to change the localization [328-334]. Rab1b, Rab4a, Rab11b, Rab2b and Rab20 are recruited to the YCV during infection of macrophages, this raises the possibility that $Y$. pestis, similar to other intracellular pathogens, encodes effector proteins that are delivered into the host cell to actively target and modify these Rab GTPases, ultimately leading to recruitment of them to the YCV. To date, conventional bacterial mutagenesis loss of function approaches have not yielded any potential bacterial effector proteins from Y. pestis required for YCV biogenesis, or that interact with Rab proteins (or Rab interacting partners), which could be explained by functional redundancy (i.e., Y. pestis encodes more than one effector protein that is sufficient to generate the YCV). However, now that I have identified Rab proteins required for the YCV biogenesis, we can use a host-directed approach to identify bacterial proteins that interact with these Rab proteins, bypassing complications of functional redundancy. Specifically, to identify potential bacterial effector proteins interacting with the Rab proteins known to be important for Y. pestis intracellular survival, I would use a protein interaction discovery tool, termed BiolD [335, 336]. BiolD is a useful tool for labeling interacting proteins, as it is an enzyme that biotinylates proteins within close proximity to the tagged protein of interest, in this case our 
Rab GTPases of interest [335]. Fusion of BiolD to individual Rab proteins could then be overexpressed in RAW264.7 cells. Upon transfection of the Rab-BiolD construct, cells would then be infected with $Y$. pestis to deliver potential bacterial effector proteins into the macrophages. Proteins (bacterial and host) in close proximity to the Rab-BiolD complex would be biotinylated. Lysis of the macrophages followed by pull down with streptavidin, would purify the biotin labeled proteins that were in proximity to the Rab protein. Using MS/MS on the purified proteins would allow for identification of the tagged proteins. The BiolD method is advantageous for two reasons: 1) Proteins in close proximity are marked, even if the interaction is transient, allowing for identification of interactions otherwise missed due to time constrained assays; 2) BiolD does not require tight interacts between the host protein and potential bacterial protein that might be disrupted through traditional pull down assays. While a temporal aspect can be incorporated to finely dissect event timelines, all interactions, whether early or late in the infection process, can be identified using BiolD and harvesting at a later time post-infection. BiolD results could be further validated by conventional in vitro biochemical protein-protein interaction assays and characterization of the YCV biogenesis process during infection with bacterial mutants in potential Rab interacting proteins.

One caveat to this approach is that if $Y$. pestis effector proteins do not directly interact with the Rab protein of interest tagged to BiolD, but instead with a host Rab interacting protein, those interactions would not be identified. If the BiolD approach fails to identify bacterial proteins interacting with the Rab proteins of interest, an alternative approach would be to determine if specific Rab interacting partners are required for YCV biogenesis and/or intracellular survival. This could be accomplished using RNAi for these proteins as I have shown for the individual Rab proteins. If RNAi identifies specific Rab interacting partners required for $Y$. pestis intracellular survival, BiolD using those Rab interacting partners as bait could be used to identify potential bacterial proteins that they are interacting with.

\subsubsection{Hypothesis 2: Y. pestis binds to a specific receptor to avoid phagosome maturation.}

While bacterial effector proteins mediate phagosome maturation during infection with other bacteria, it is possible that $Y$. pestis may not encode effector proteins to alter vesicular trafficking 
through targeting of Rab proteins. Alternatively, ligation to a receptor, or manipulation of receptor adaptors could dictate intracellular trafficking events. For example, Chlamydia pneumoniae interacts with the epidermal growth factor receptor (EGFR) to subvert lysosomal degradation and directly enter into the recycling pathway [337]. Since we have shown that the YCV is remodeled to resemble a recycling endosome, this raises the possibility that $Y$. pestis could use a receptor mediated mechanism similar to $C$. pneumoniae to enter the recycling pathway. However, what receptor mediates $Y$. pestis phagocytosis by macrophages is unclear.

Receptor ligation is not a novel concept in $Y$. pestis biology, several receptors have been identified that are expressed by innate immune cells and bind to different surface expressed antigens on Y. pestis. Formyl peptide receptor 1 (FPR1) is the most recent innate immune cell receptor shown to bind to Y. pestis [338]. Binding to FPR1 via T3SS tip protein LcrV, enhances delivery of Yop effector proteins into the host cell cytosol, and is detrimental to the innate immune cells due to increased targeting efficiency by the T3SS [338]. However the T3SS is not expressed upon transmission from the flea vector into the mammalian host, when Y. pestis is first interacting with macrophages and bacteria lacking the pCD1 plasmid, which encodes the T3SS, are still able to generate a YCV and survive within macrophages [102]. Therefore, FPR1 ligation to LcrV is unlikely to mediate uptake of $Y$. pestis by macrophages or play a role in YCV biogenesis.

Other known host receptors which bind to $Y$. pestis antigens expressed upon transmission from the flea vector are CD205 (DEC-205) and CD209 (SIGN-R1) [52, 339, 340]. CD205 and CD209 bind to Pla and the core of LPS, respectively, but whether CD205 ligation to Pla or CD209 ligation to LPS impacts vesicular trafficking within macrophages is not known. Blocking either receptor diminishes phagocytosis of Y. pestis. However, only partially, indicating another receptor, or perhaps binding to multiple receptors dictates Y. pestis uptake by macrophages [339].

TLR4 is also an intriguing possibility as a potential receptor for mediating $Y$. pestis phagocytosis. TLR4 is endocytosed upon ligation to LPS and returned to the plasma membrane through the recycling pathway, similar to EGFR. Y. pestis is known to modulate TLR4 signaling, by changing LPS acylation and by inhibiting MyD88 signaling [49, 51, 301]. Interestingly, TLR4 can 
traffic to different intracellular compartments, such as early endosomes, phagosomes, Rab11 positive recycling vesicles, and multi-vesicular bodies depending on the signaling pathway activated (reviewed in [341]). Inhibition of TLR4 signaling, through disruption of adaptor protein recruitment modulates trafficking of TLR4 within cells [342, 343]. Interestingly, binding of $E$. coli by TLR4 induces not only signaling cascades, but phagocytosis specifically by ligation of LPS with TLR4 through the adaptor protein TRAM $[343,344]$. In turn, TRAM interacts with Rab11 family of interacting proteins 2 (FIP2) [343]. Manipulation of TLR4 ligation to bacterial bound LPS and interaction with adaptor proteins presents a point where $Y$. pestis could exploit host cell signaling to its advantage. Both TLR4 and EGFR can be found in Rab11 positive endosomes for recycling back to the plasma membrane. Therefore, targeting of TLR4 (or EGFR) may allow pathogens to avoid phagolysosomal fusion. We do not clearly understand how $Y$. pestis induces uptake by macrophages, but because Y. pestis LPS is known to modify TLR4 signaling, it raises the possibility that binding to TLR4 may contribute to YCV biogenesis independent of direct bacterial effector recruitment. Knockdown of TLR4, EGFR, and other potential receptors (i.e CD205 and CD209), or TLR4 associated proteins (CD14 and MD-2) could be used to identify if Y. pestis is binding and being taken up through a receptor dependent pathway to modulate $Y$. pestis intracellular trafficking. Knockdown could be combined with other assays to evaluate whether fusion with lysosomes is impacted, or association with markers of autophagy and YCV expansion.

\section{$\underline{5.2 \text { How are neutrophil antimicrobial responses altered during Y. pestis infection? }}$}

The data presented in Chapter 4 enhances our understanding of how Y. pestis inhibits release of neutrophil granules and $\mathrm{LTB}_{4}$ production. However, through my work studying neutrophil responses to $Y$. pestis, I have accumulated additional observations that have raised questions for further exploration. The following paragraphs discuss these questions and potential future directions of study to better understand Y. pestis-neutrophil interactions.

\subsubsection{Which host proteins do Yop effectors interact with in human neutrophils to inhibit signaling?}

Much of our current understanding of how Y. pestis Yop effector proteins impact host signaling pathways comes from studies using macrophages, with little verified in neutrophils. 
Additionally, several Yops have been shown to modify multiple host proteins in vitro, but whether all or only one of these proteins is actually targeted in vivo is unclear. Moreover, many studies of Yop functions have used Y. pseudotuberculosis or $Y$. enterocolitica, not Y. pestis [226, 285]. Although the T3SS and Yop effector proteins are highly conserved between the three species, there are some known differences that could influence outcomes [303]. Cumulatively, little evidence has been published that directly demonstrates which host proteins are directly targeted by Yops during Y. pestis infection of neutrophils. Better understanding how Y. pestis disrupts host cell signaling could potentially assist in mapping neutrophil signaling pathways that may differ from macrophages.

To specifically identify host proteins that are targeted by $Y$. pestis Yop effectors within neutrophils, I would use the BiolD system described in Section 5.1.1. Briefly individual Yop effectors would be fused to BiolD and complemented into Y. pestis lacking the WT copy of the Yop protein (a copy lacking a Yop effector could be transformed into Y. pestis to act as a negative control and $\mathrm{YopH}$, known to interact with FAC proteins in neutrophils, would act as confirmation of assay viability). Human neutrophils would then be infected, cells lysed, and biotinylated proteins purified, and identified by MS/MS. Identifying all the potential interactions of Yop effectors with host proteins would be invaluable to understand how $Y$. pestis inhibits neutrophil responses.

\subsubsection{How does $Y$. pestis inhibit the synthesis of $\mathrm{LTB}_{4}$ ?}

Production and release of $\mathrm{LTB}_{4}$ by neutrophils is an important factor for mounting an effective inflammatory response against bacteria [201, 203]. Synthesis of LTB4 requires 1) activation of receptor signaling, 2) activation of $\mathrm{Ca}^{2+}$ flux and/or MAPKs, 3) liberation of arachidonic acid, 4) phosphorylation of 5-LO and/or association with $\left.\mathrm{Ca}^{2+}, 6\right)$ translocation of 5-LO to the nucleus/other membranous compartment, 7) association with accessory proteins (i.e. FLAP), and 8) activity of $\mathrm{LTA}_{4} \mathrm{H}$ (Figure 1-3) $[189,191,194,195,345]$. While these steps are known to be important for $\mathrm{LTB}_{4}$ production, there remains aspects that are unknown. Translocation of 5-LO from the cytosol to a lipid membrane is a hallmark of active 5-LO. However, the mechanism that drives 5-LO to translocate to membranes remains unclear. 5-LO associates with CLP and Grb2, two actin interacting proteins, and yet to date a direct role for the cytoskeleton in 5-LO translocation has not 
been demonstrated. Additionally, phosphorylation by MAPKs and association with $\mathrm{Ca}^{2+}$ are also thought to be important for translocation to a membrane, but have not been fully elucidated. The lack of knowledge regarding what is critical for 5-LO translocation/activation presents an area for further exploration. My findings have pointed to the importance of ERK activation and a dynamic cytoskeleton, yet what remains unknown is which particular step(s) in the synthesis pathway is/are directly inhibited by the Yop effector proteins to inhibit production of $\mathrm{LTB}_{4}$ ?

5-LO activity is regulated by the MAPK pathway, association with calcium, and requires translocation. All of these signal pathways are known targets of Yop effector proteins. Therefore, activation of 5-LO is likely a key regulatory point for inhibition of $\mathrm{LTB}_{4}$ production by $\mathrm{Y}$. pestis. Previous studies have shown that ERK phosphorylation of 5-LO enhances activation and translocation [189, 191, 345, 346]. My work has shown that ERK phosphorylation is required for $\mathrm{LTB}_{4}$ production in response to Y. pestis. Thus, it is likely that inhibition of ERK phosphorylation by YopJ inhibits phosphorylation of 5-LO, and ultimately prevents translocation to a lipid membrane. To determine whether inhibition of ERK phosphorylation impairs phosphorylation of 5-LO during $Y$. pestis infection, western blotting could be performed using antibodies specific to un-phosphorylated and phosphorylated 5-LO during infection with Y. pestis $T 3^{(+)}$, Y. pestis expressing YopJ only, or $Y$. pestis $\mathrm{T3}^{(-)}$. Based on my results from Chapter 4 , I expect that 5-LO phosphorylation will occur in cells infected with $Y$. pestis $\mathrm{T}^{(-)}$but not those infected with $Y$. pestis $\mathrm{T}^{(+)}$or $Y$. pestis expressing only YopJ. Addition of ERK1/2 chemical inhibitor (U0126) to cells infected with Y. pestis T3 ${ }^{(-)}$will be able to determine if this inhibition is ERK specific. Whether 5-Lo phosphorylation by ERK is required for translocation to the nuclear membrane/lipidosome is not clear, but microscopy studies to evaluate cellular localization of 5-LO in relation to FLAP, the nucleus, or lipidosomes could be used in infected cells to determine if $Y$. pestis inhibits this required step in LTB 4 synthesis.

While ERK mediated phosphorylation of 5 -LO is important for 5 -LO activation, $\mathrm{LTB}_{4}$ production was also inhibited by Yop effector proteins that target cytoskeleton rearrangement, but independent of MAPK signaling. Translocation of 5-LO upon activation is known to occur, although no conclusive evidence as to how the translocation occurs has been shown. Currently, there are three factors which have been proposed to drive translocation of 5-LO, phosphorylation, 
association with $\mathrm{Ca}^{2+}$, and movement via the actin cytoskeleton. The first two factors, phosphorylation and association with $\mathrm{Ca}^{2+}$ are thought to increase the affinity between 5-LO and phosphatidylethanolamine (PE) to drives 5-LO to membranous compartments rich in PE [191, 345]. Whether the increased affinity for PE is sufficient to drive translocation has not been determined. Moreover, whether both phosphorylation and association with $\mathrm{Ca}^{2+}$ are required, or if one or the other is sufficient, remains undetermined. A more likely scenario, is that active transport by the cytoskeleton assists in translocation and is enhanced by the increase in affinity for PE. The requirement for cytoskeleton rearrangement is supported by my finding that four Yop effectors which target the cytoskeleton can inhibit translocation, in addition to data showing 5-LO interacts with CLP and Grb2, actin binding proteins [186, 345]. Based on this information, I developed the hypothesis that $Y$. pestis inhibits $\mathrm{LTB}_{4}$ production by disrupting not only ERK phosphorylation, but also cytoskeletal rearrangement and $\mathrm{Ca}^{2+}$ flux, thereby preventing translocation of 5-LO from the cytosol to membranes rich in AA. To understand the importance of $\mathrm{Ca}^{2+}$ flux and the role of cytoskeletal rearrangement for 5-LO activity two different approaches would need to be implemented. The first approach would aim to understand the role of Yop effectors which target the actin cytoskeleton. To do this, I would use antibodies specific to 5-LO, FLAP, and stains specific to the nuclear membrane and lipidosomes in combination with phalloidin, which binds to the actin cytoskeleton. Using these markers, I would infect neutrophils with $Y$. pestis $\mathrm{T}^{(+)}$, Y. pestis independently expressing each of the four Yop effector proteins (YpkA, YopE, YopH, and YopT), or Y. pestis $\mathrm{T}^{(-)}$with or without latrunculin A pre-treatment. I would then assess 5-LO colocalization to FLAP/membrane compared to UT neutrophils as a negative control. I expect that infection with $Y$. pestis $\mathrm{T}^{(-)}$would result in co-localization to $\mathrm{FLAP} /$ membrane. I also expect that WT Y. pestis would not result in co-localization, and a similar result for the four Yop effector protein infections, as well as $T 3^{(-)}$when latrunculin $A$ is used as a pre-treatment. Results similar to those stated would confirm that translocation via the cytoskeleton is important for 5-LO activity. To further define the cytoskeletal signaling pathway important for 5-LO translocation, I would use inhibitors specific for Rac or RhoA in place of latrunculin A. 
The second approach would aim to delineate the role of $\mathrm{Ca}^{2+}$ in translocation and activity of 5-LO during Y. pestis infection, focusing specifically on YopH. YopH inhibits signaling through the FAC and in so doing, inhibits $\mathrm{Ca}^{2+}$ flux in neutrophils. Therefore, it is likely that inhibition of $\mathrm{Ca}^{2+}$ flux by $\mathrm{YopH}$ impacts $\mathrm{LTB}_{4}$ production as well. For this approach, cells would be infected with $Y$. pestis $\mathrm{T}^{(+)}, Y$. pestis $\mathrm{T} 3^{(-)}$, or $Y$. pestis expressing only $\mathrm{YopH}$ and treated with calcium ionophores or calcium chelators. Localization of 5-LO would be assessed as described above, in addition to collection of supernatants to determine LTB4 production, described in the methods section. If the activity of $\mathrm{YopH}$ on $\mathrm{Ca} 2+$ flux is responsible for inhibit translocation of 5-LO, then it would be expected that the simple addition of a $\mathrm{Ca}^{2+}$ chelator during $Y$. pestis $\mathrm{T}^{(-)}$infection would cause 5LO not to translocate to FLAP, and $\mathrm{LTB}_{4}$ not to be produced. Conversely, utilization of a $\mathrm{Ca}^{2+}$ ionophore concomitantly with $\mathrm{Y}$. pestis $\mathrm{T}^{(+)}$or $\mathrm{Y}$. pestis expressing only $\mathrm{YopH}$ would result in translocation of 5-LO to FLAP and production of $\mathrm{LTB}_{4}$. Together, these studies will identify the specific molecular mechanisms used by $Y$. pestis to inhibit $\mathrm{LTB}_{4}$ synthesis.

\subsubsection{What impact does YopJ inhibition of p38 phosphorylation have on neutrophil responses?}

While the MAPK pathway is required for production of $\mathrm{LTB}_{4}$, many other neutrophil antimicrobial responses also rely upon the MAPK pathway for activation. Some antimicrobial responses that depend on MAPK signaling include: production of the respiratory burst, granule release, and cytokine production [159, 216, 313, 347]. Signaling through the MAPK pathway is also not a simple, linear cascade, but rather, a complex, multi-branched, differentially activated network that works in a coordinated way to enact specific responses in a regulated manner [216, 313, 348]. I showed that the effector protein YopJ encoded by Y. pestis is sufficient to inhibit ERK signaling and block production of $\mathrm{LTB}_{4}$. Furthermore, I have also shown that p38 is also blocked by the activities of $Y$. pestis, but that inhibition of p38 phosphorylation was not required for inhibition of $\mathrm{LTB}_{4}$ production (Figure 4-8). Phosphorylation of p38 is blocked by the action of YopJ (Figure 5-4), however the benefit of blocking p38 phosphorylation in neutrophils to $Y$. pestis has not been elucidated to date. Signaling through p38 is essential to activation of the respiratory burst and granule release $[154,159,325,347,349]$, two neutrophil antimicrobial responses inhibited by the Y. pestis T3SS [166]. The direct inhibition of $p 38$ was not demonstrated as the key factor regulating 
these responses to $Y$. pestis infection, and the question remains whether YopJ inhibition of p38 phosphorylation is directly responsible for inhibition of the respiratory burst and degranulation. To determine whether Y. pestis, and specifically YopJ inhibit p38 phosphorylation in neutrophils to directly inhibit the respiratory burst and degranulation, I would use a combination of chemical inhibitors and Y. pestis strains to infect neutrophils and then monitor production of ROS and release of granules. Briefly, I would pretreat neutrophils with or without an inhibitor of p38 and subsequently leave cells untreated or infect with either $Y$. pestis $T 3^{(+)}$, Y. pestis $T 3^{(-)}$, or $Y$. pestis only expressing YopJ. I would confirm inhibition of p38 is occurring via Western blot, then I would assay granule release as outlined in the methods section, or measure ROS production with a luminol assay. If inhibition of $\mathrm{p} 38$ phosphorylation is required to inhibit these processes, I expect to observe reduced ROS and granule release by $Y$. pestis $\mathrm{T}^{(-)}$infected neutrophils in the presence of the $\mathrm{p} 38$ inhibitor. However, especially for degranulation two of three pathways may need to be inhibited by Yop effector proteins in order to fully suppress granule release. Therefore, inhibition of degranulation may only be observed when a Y. pestis strain expressing only YopE and YopJ, or YopH and YopJ is used. If addition of the p38 inhibitor does not inhibit ROS or degranulation, then a second inhibitor of Rac2 or $\mathrm{Ca}^{2+}$ flux may need to be used.

A

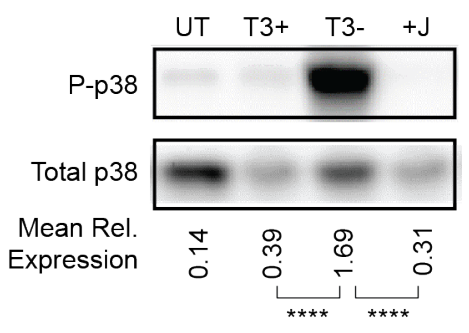

B

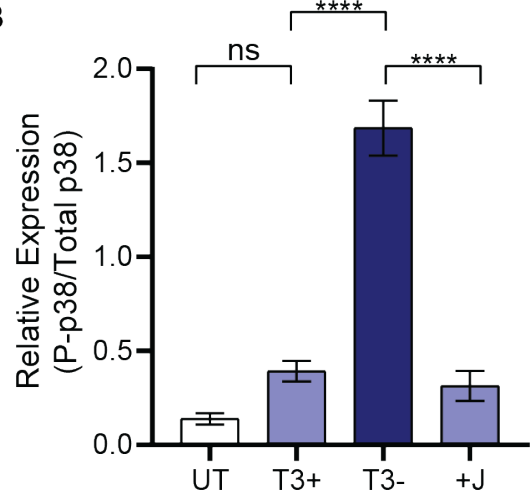

Figure 5-4: YopJ inhibits p38 phosphorylation.

Human neutrophils $\left(8 \times 10^{6}\right)$ were infected with Y. pestis KIM1001 with or without the pCD1 plasmid encoding the T3SS (T3+ or T3-, respectively), or expressing only yopJ $(+\mathrm{J})$; $\mathrm{MOI}=100$. (A) Representative western blot with mean relative expression of phosphorylated or un-phosphorylated p38 during infection with indicated strains. (B) Mean relative expression of phosphorylated p38 
during infection with indicated Y. pestis strains. Y. pestis $\mathrm{KIM}_{1001}=\mathrm{T} 3+; \mathrm{KIM} 1001 \mathrm{~T}^{(-)}=\mathrm{T} 3-;$ KIM1001 expressing only yopJ $=+\mathrm{J}$; Uninfected $=\mathrm{UT}$. Mean \pm SEM from 3 biologically independent experiments. One-way ANOVA with Sidak's post-hoc; ns= not significant, ${ }^{* * *}, \mathrm{P}<0.0001$.

\subsubsection{Does $Y$. pestis inhibit release of NETs by neutrophils?}

In addition to the antimicrobial mechanisms I have previously discussed, neutrophils can also restrict bacterial growth by limiting the availability of metals through release of calprotectin, to sequester $\mathrm{Zn}, \mathrm{Mn}$, and Fe from pathogens [350]. This is part of the host response to infection termed nutritional immunity. Mechanisms used by $Y$. pestis to overcome nutritional immunity are key to infection and understanding these mechanisms is of great interest in our lab. Calprotectin composes $\sim 40 \%$ of the cytosolic protein in neutrophils, and is normally released in response to infection independent of granule release [157].

Having observed that Y. pestis actively inhibits other exocytic processed in neutrophils (i.e. degranulation) I also predicted that that $Y$. pestis inhibits release of calprotectin by neutrophils. To test this hypothesis human neutrophils were treated with Y. pestis KIM1001 with or without the T3SS (T3+ or T3-), E. coli, PMA (positive control), or co-treated with Y. pestis expressing the T3SS (T3+) and PMA for $30 \mathrm{~min}, 1 \mathrm{~h}$, or $3 \mathrm{~h}$. Release of calprotectin into the supernatant was measured by ELISA (Figure5-5). At 30 min post-infection, calprotectin was not detected in any of the samples collected. However, by $1 \mathrm{~h}$ post-infection, PMA-treated neutrophils began to release calprotectin slightly over that measured for UT, while the other treatments remained below UT levels. By $3 \mathrm{~h}$ post-infection, PMA treatment induced release of calprotectin to significantly higher levels ( $p$; $>0.001$ ). Intriguingly, co-treatment with $Y$. pestis $\mathrm{T}^{(-)}$and PMA did not result in increased calprotectin release similar to that seen for PMA alone at $3 \mathrm{~h} \mathrm{PI} \mathrm{(Figure} \mathrm{5-5A).} \mathrm{These} \mathrm{data} \mathrm{indicate}$ that at least in vitro, Y. pestis is able to inhibit calprotectin release by human neutrophils. Infection with $Y$. pestis $T 3^{(-)}$by $3 \mathrm{~h}$ post infection also did not result in release of calprotectin over that observed for UT, and the level of calprotectin is similar to $\mathrm{Y}$. pestis $\mathrm{T} 3^{(+)}$at $3 \mathrm{~h}$ post infection. Taken together, this data indicated release of calprotectin from human neutrophils is being inhibited by $Y$. pestis in a T3SS independent manner. 

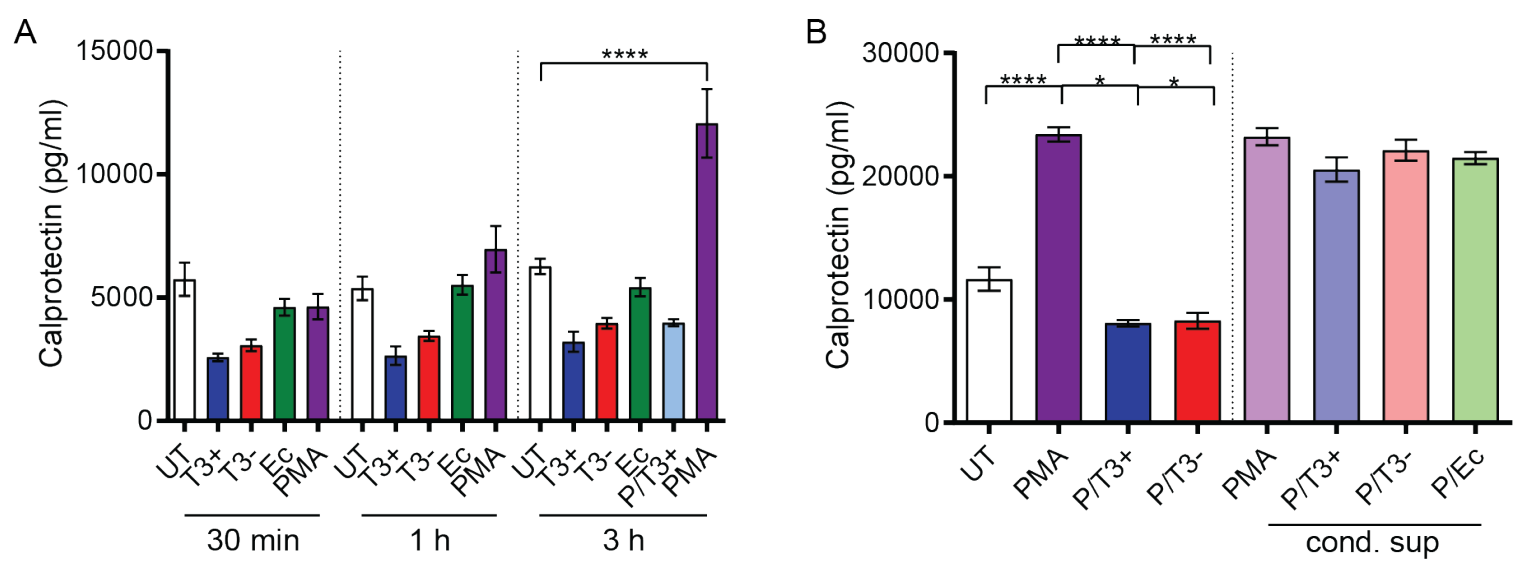

Figure 5-5: Y. pestis inhibits release of calprotectin from human neutrophils.

Human neutrophils $\left(4 \times 10^{6}\right)$ were infected with Y. pestis KIM1001 with or without the pCD1 plasmid encoding the T3SS (T3+ or T3-, respectively), E. coli, or treated with PMA and infected with Y. pestis at the same time; $\mathrm{MOI}=100$. (A) Release of Calprotectin was measured after $30 \mathrm{~min}, 1 \mathrm{~h}$, or 3h after infection in supernatant. (B) Calprotectin in supernatant from PMA post treated with $Y$. pestis for $3 \mathrm{~h}$ or supernatant from neutrophils that were mixed with an equivalent number of $Y$. pestis or $E$. coli as was in the original cell infection $(3 \mathrm{~h})$. Mean \pm SEM from 3 biologically independent experiments. One-way ANOVA with Dunnett's post hoc test to UT or PMA. ${ }^{* *}, P<0.01$; ${ }^{* * * *}, P<0.0001$.

The absence of calprotectin in the culture supernatant in both $Y$. pestis $\mathrm{T} 3^{(+)}$and $\mathrm{T} 3^{(-)}$ indicated that calprotectin levels are controlled by a T3SS independent mechanism. One possible mechanism may be that the bacterium does not inhibit release, but degrades calprotectin once released. $Y$. pestis encodes several proteases that may be contributing to degradation of calprotectin. In order to test whether Y. pestis uses a protease to degrade calprotectin, supernatant from the PMA $3 \mathrm{~h}$ treated neutrophils was incubated at $37^{\circ} \mathrm{C}$ for $3 \mathrm{~h}$, either without bacteria, with an equivalent amount of $Y$. pestis $\left(\mathrm{T}^{(+)}\right.$or $\left.\mathrm{T} 3^{(-)}\right)$or with $E$. coli to the bacteria to volume ratio initially incubated with neutrophils. Incubation of PMA supernatant with Y. pestis $\mathrm{T} 3^{(+)}$, Y. pestis $\mathrm{T3}^{(-)}$, or $E$. coli did not result in a reduction of calprotectin present (Figure 5-5B). This observation suggests that $Y$. pestis does not degrade calprotectin released into the supernatant through the activity of a protease, and instead suggests that calprotectin release is inhibited by $Y$. pestis. 
The leading mechanism proposed for calprotectin release is through a mechanism referred to as Neutrophil extracellular trap (NET) release or NETosis [157, 350, 351]. NETosis is a process where decondensed DNA is extruded by neutrophils, and is thought to be an active process to "trap" pathogens in order to limit their dissemination [178, 278]. NETosis occurs when granule proteins, such as neutrophil elastase (NE) are released into the cytosol through activation of PAD4, and subsequently enters into the nucleus [175]. NE degrades histones to de-condense the genetic material and allows for DNA extrusion into the extracellular matrix. In addition to DNA, these NETs are decorated with granule and cytosolic proteins such as elastase, myeloperoxidase, and calprotectin, which can directly kill or metabolically starve the pathogen [174].

While previous studies have reported that Y. enterocolitica and pseudotuberculosis stimulate NET release due to expression of the bacterial adhesin protein YadA [352], YadA was lost during divergence of $Y$. pestis from $Y$. pseudotuberculosis and the degree of NETosis in response to $Y$. pestis has not been clearly evaluated. However, my preliminary data demonstrating an absence of calprotectin release in response to $Y$. pestis strongly suggests that $Y$. pestis inhibits NETosis. To test this idea, I would adhere neutrophils to coverslips and infect the cells with $Y$. pestis or the enteric Yersinia. I would then use antibodies for calprotectin, neutrophil elastase, and myeloperoxidase to measure NET release. If $Y$. pestis inhibits NETosis, I expect to observe diminished levels of $\mathrm{CP}, \mathrm{NE}$, and MP around neutrophils infected with $\mathrm{Y}$. pestis compared to the enteric Yersinia and the positive control, PMA. However, if Y. pestis does not suppress NETosis, this would indicate that calprotectin is being released by a mechanism independent of NETosis.

\subsubsection{Does the exosome profile released by neutrophils change upon infection with Y. pestis?}

My data strongly supports that $Y$. pestis is capable of inhibiting exocytic pathways by human neutrophils, including the release of granules and calprotectin. Exosomes are small vesicles released by cells through fusion of multivesicular bodies to the plasma membrane and can contain proteins, RNAs, and lipids [353-355]. These vesicles have increasingly been the topic of host cell communication studies [193, 194, 356]. Mounting evidence shows that exosomes are released with contents to relay messages to other cells nearby, or even ones far away [353, 356]. Incorporating DNA, RNA, proteins, or lipids within a membrane bound envelope increases molecular stability and 
allows for enhanced traversing of distances to relay information that would otherwise quickly be degraded in the extracellular milieu [193, 194, 354]. Release of exosomes is dependent on the actin cytoskeleton [357], a known target of Yop effector proteins [72, 74-76, 358]. However, since Y. pestis has been shown to inhibit other arms of exocytosis, it is intriguing to consider that $Y$. pestis may also inhibit the release of exosomes to curtail innate immune cell responses to infection.

The first question that would need to be answered to test this hypothesis is whether $Y$. pestis completely inhibits exosome release similar to degranulation? To answer this question, I would infect human neutrophils with $M$. bovis as a positive control [359], Y. pestis $\mathrm{T}^{\mathrm{C}^{(+)}}$or $Y$. pestis $\mathrm{T} 3^{(-)}$, isolate exosomes using ultracentrifugation, and quantify the amount of exosomes released by each sample using an ELISA. If $Y$. pestis inhibits exosome release, I would expect fewer exosomes from neutrophils infected with $Y$. pestis $T 3^{(+)}$compared to $M$. bovis. Moreover, if exosome inhibition is dependent on the T3SS, then the concentration of $Y$. pestis $\mathrm{T}^{(-)}$exosomes would be significantly greater than $Y$. pestis $\mathrm{T}^{(+)}$

The second question that would need to be explored is if $Y$. pestis infection modifies exosome content (this could occur in addition to inhibition of release or separately). To determine if the exosome content differs between UT neutrophils, neutrophils infected with a non-pathogenic bacteria such as $E$. coli, and neutrophils infected with $Y$. pestis, I would infect human neutrophils and then isolate exosomes as described above. I would standardize the number of exosomes analyzed and then use lipidomics, proteomics, and transcriptomics to analyze differences between the samples. If differences are observed, then the impact of these exosomes on innate immune cell function could be explored. This study could be further extended to in vivo Y. pestis infection of mice, where exosomes could be isolated from Y. pestis $\mathrm{T}^{(+)}$and $\mathrm{T} 3^{(-)}$infected mouse tissues, such as the lungs or lymph nodes, If the exosome profile is different between $\mathrm{T} 3^{(+)}$and $\mathrm{T} 3^{(-)}$infected mice, it would be interesting to determine if exosomes from the $Y$. pestis $T 3^{(-)}$infected mice could be transferred to naïve mice to induce control of the Y. pestis $\mathrm{T}^{(+)}$infection. 


\subsection{What is the impact of $\mathrm{LTB}_{4}$ signaling on plague?}

\subsubsection{Does exogenous addition of $\mathrm{LTB}_{4}$ enhance the degradative capacity of innate immune}

cells?

$\mathrm{LTB}_{4}$ is critical to controlling infection caused by many pathogens [203, 208-210,360]. The importance of $\mathrm{LTB}_{4}$ in controlling infection is due to the ability of $\mathrm{LTB}_{4}$ to stimulate enhanced phagocytosis, improved microbial killing, and trafficking of innate immune cells to the site of infection, via establishment of a chemotactic gradient [203, 206]. Exogenous addition of LTB4 enhances the innate immune response and can foster immune cell mediated clearance of infection [198]. Since I have shown that $Y$. pestis actively inhibits LTB release from human neutrophils, and $\mathrm{LTB}_{4}$ influences immune cell antimicrobial responses, I hypothesize that exogenous addition of $\mathrm{LTB}_{4}$ to innate immune cells (i.e. neutrophils and macrophages) would enhance killing of $Y$. pestis in vitro. To test this, human macrophages and neutrophils would be isolated and adhered to separate 96-well plates. I would add exogenous $\mathrm{LTB}_{4}$ (a range of concentrations) to the phagocytic cells either prior to or at the time of $Y$. pestis infection, and survival of $Y$. pestis would be monitored over time. The ability to kill Y. pestis would be compared to the cells which did not receive LTB4. Based on the ability of $\mathrm{LTB}_{4}$ to enhance the antimicrobial activities of phagocytic cells, I would expect that the macrophages and neutrophils treated with $\mathrm{LTB}_{4}$ and infected with $Y$. pestis would show an enhanced killing of $Y$. pestis, over that observed for cells which were not treated with LTB4. To verify the activity is attributable to $\mathrm{LTB}_{4}$, chemical inhibitors of $B L T 1$, the primary receptor could be used to inhibit signaling through the primary receptor, or inhibit BLT1 signaling using blocking antibodies.

\subsubsection{Could administration of $\mathrm{LTB}_{4}$ enhance host resistance to pneumonic plaque?}

Although there is much that can be learned from in vitro studies with immune cells, it is impossible to fully model the complex and dynamic interactions that occur between multiple cell types as occurs in vivo. The murine model of plague is the most commonly used laboratory model used to study Y. pestis virulence, and disease in mice recapitulates many of the hallmarks of plague observed in human, included delayed inflammation, infection of draining lymph nodes, bubo 
formation, and necrosis of tissue [85, 110]. Therefore, while I have shown inhibition of neutrophil responses in vitro, in the future it would be desirable to translate these studies into an in vivo model like the mouse to better understand the consequences of these interactions in the context of the entire immune system.

The inhibition of $\mathrm{LTB}_{4}$ by $Y$ pestis in vitro suggests $\mathrm{LTB}_{4}$ should not be released during in vivo infection either. However, neutrophils are not the only cell type capable of producing $\mathrm{LTB}_{4}$, other immune cells, such as macrophages and mast cells are also capable of producing $\mathrm{LTB}_{4}$, which many release $\mathrm{LTB}_{4}$ in vivo [282, 322]. To ascertain if $\mathrm{LTB}_{4}$ is produced during in vivo infection, mice were infected via intranasal instillation during a pilot study. Briefly $Y$. pestis with or without the T3SS (T3+ or T3-, respectively), or the negative control, PBS, was instilled into the mouse lungs. BALF was collected at $12 \mathrm{~h} \mathrm{PI}$, concentrated using a $\mathrm{C} 18$ column, re-suspended in $\mathrm{H}_{2} \mathrm{O}$, and $\mathrm{LTB}_{4}$ was measured. Infection of mice via intranasal instillation of either $Y$. pestis strain did not result in a significant increase in $\mathrm{LTB}_{4}$ released (Figure 5-6C). Together, these data suggest $Y$. pestis infection does not release $\mathrm{LTB}_{4}$, but additional time points should be examined to completely characterize the $\mathrm{LTB}_{4}$ response during pneumonic plague. A caveat to this data is that while $\mathrm{LTB}_{4}$ does not appear to be released upon infection of mice with $Y$. pestis, infection with a pathogen known to induce release of $\mathrm{LTB}_{4}$ in the lungs, such as Klebsiella pneumoniae, would provide further evidence that $L T B_{4}$ can be released, but that $Y$. pestis does not stimulate release or actively inhibits LTB4 release $[198,203]$.

However, these preliminary data support the hypothesis that inhibition of the LTB4 mediated response contributes to the non-inflammatory environment associated with early plague. To understand whether exogenous addition of $\mathrm{LTB}_{4}$ increases resistance to WT Y. pestis during in vivo infection, I would infect mice with WT Y. pestis that have received exogenous LTB4. (dose and timing of administration could be titrated), monitoring bacterial proliferation and host survival as a measurement of efficacy. If inhibition of $\mathrm{LTB}_{4}$ contributes to the non-inflammatory environment associated with pneumonic plague, I expect that the mice treated with LTB 4 would have less $Y$. pestis CFU counts and would survive longer than the mice that were not treated with $\mathrm{LTB}_{4}$. One caveat to this approach is that $\mathrm{LTB}_{4}$ is highly inflammatory. Treatment of mouse lungs with $\mathrm{LTB}_{4}$ 
could cause detrimental inflammation over that beneficial to clearing the $Y$. pestis infection, which could lead to damage of the lung tissue, pneumonia, and death. Careful titration would be needed to determine an effective dose, that doesn't stimulate deleterious effects, within the mouse lungs.

\section{$\underline{5.4 \text { Do murine neutrophils release } \mathrm{LTB}_{4} \text { in response to } Y \text {. pestis? }}$}

While mice are widely used to model human infection, it is widely recognized that human and mouse neutrophils differ in their responses to bacteria, and I described some of these differences in Chapter 1. Data published near the when I published my data on degranulation indicates that $Y$. pestis is also able to inhibit degranulation in murine neutrophils [361]. However, the $\mathrm{LTB}_{4}$ response by murine neutrophils to $Y$. pestis has not been explored. Therefore, to begin to answer this question, bone marrow derived neutrophils (BMNs) from C57BI/6 mice were isolated and infected with Y. pestis KIM1001 with or without the T3SS (T3+ and T3-, respectively). After 30 min of infection, supernatant was collected and release of $\mathrm{LTB}_{4}$ was measured (Figure 5-6). As observed for human neutrophils, infection with $Y$. pestis $\mathrm{T3}^{(+)}$did not result in significant $\mathrm{LTB}_{4}$ secretion over untreated BMNs. Surprisingly, BMNs infected with Y. pestis $\mathrm{T}^{(-)}$also did not release $\mathrm{LTB}_{4}$. This lack of $\mathrm{LTB}_{4}$ production was likely not due to the maturity of BMNs compared to peripheral neutrophils, since infection with $E$. coli resulted in production of LTB (Figure 5-6A). These findings with murine BMNs are in contrast to my previous studies with human neutrophils, and raise interesting questions about potential differences in $\mathrm{LTB}_{4}$ signaling between mouse and human neutrophils that should be explored further.

Generally, mice produce a more toleragenic response to infection than humans, which may be why $\mathrm{LTB}_{4}$, an inflammatory mediator, is released by human neutrophils and not murine neutrophils in response to $Y$. pestis [218]. The lack of a $L T B$ response in murine BMNs compared to human neutrophils implies a difference exists in the host cell receptor recognition or downstream signaling from the receptor. For $Y$. pestis infection specifically, several differences between human and murine receptors have previously been identified, such as recognition of $Y$. pestis LPS $[43,50$, 338]. Production of $\mathrm{LTB}_{4}$ is dependent on signaling from receptors to the MAPK pathway and $\mathrm{Ca}^{2+}$ flux. Therefore, to better understand how murine neutrophils respond to $Y$. pestis infection versus human neutrophils, I would begin by looking at whether signaling through the MAPK pathway is 
occurring in BMNs similar to what I have observed for human neutrophils (i.e. Y. pestis $\mathrm{T} 3^{(-)}$ stimulates phosphorylation of ERK and p38). I would also assess the role of $\mathrm{Ca}^{2+}$ flux in murine and human neutrophils and the impact on production of $\mathrm{LTB}_{4}$, to determine if the pathway is functioning in a similar manner in both cell types. To do this, I would use a $\mathrm{Ca}^{2+}$ ionophore to flood the neutrophils and assay $\mathrm{LTB}_{4}$ production. Subsequently, to understand transcriptional differences occurring between the human and murine neutrophils in response to $Y$. pestis infection, I would perform RNAseq on highly purified neutrophils from humans and mice to directly compare the differential responses enacted by each cell type to $Y$. pestis infection. While the mechanisms may differ, it is important to stress that $Y$. pestis is able to evade $\mathrm{LTB}_{4}$ synthesis by both murine and human neutrophils. The ability to evade $\mathrm{LTB}_{4}$ synthesis supports my central hypothesis that inhibition of the normal $\mathrm{LTB}_{4}$ response to infection contributes to the ability of $Y$. pestis to establish a non-inflammatory environment.

These preliminary data indicate that murine neutrophils are unable to mount a LTB 4 response to $Y$. pestis, which also raises the question of whether this is specific for $Y$. pestis, or applies to the enteric Yersinia pathogens. To begin to explore this question, I infected BMNs with Y. pseudotuberculosis and Y. enterocolitica and compared the $\mathrm{LTB}_{4}$ response to $Y$. pestis or E. coli (Figure 5-6B). Like Y. pestis, infection with Y. pseudotuberculosis did not result in increased release of $\mathrm{LTB}_{4}$ from the BMNs. However, infection with $Y$. enterocolitica did cause an increase in $\mathrm{LTB}_{4}$ release over UT, although not to the level E. coli induced. To determine whether Y. pestis and $Y$. pseudotuberculosis actively inhibit release of $\mathrm{LTB}_{4}$ from BMNs, or if release is simply not stimulated, neutrophils were co-infected with E. coli and each Yersinia species expressing the T3SS (+/Ec). Co-infection of BMNs released $\mathrm{LTB}_{4}$ to a similar level as that observed for $E$. coli alone, suggesting Y. pestis and Y. pseudotuberculosis do not actively inhibit release of LTB from BMNs, but rather they do not stimulate release (Figure 5-6B).

While $Y$. pestis and $Y$. pseudotuberculosis do not trigger release of $\mathrm{LTB}_{4}$, $Y$. enterocolitica and E. coli do trigger release from mouse neutrophils. Some factor seems to have been lost or gained during the divergence of $Y$. pseudotuberculosis and $Y$. enterocolitica from their common ancestor, that is responsible for the difference in $\mathrm{LTB}_{4}$ response observed between human and 
mouse neutrophils. To understand what factor is different between $Y$. pseudotuberculosis and $Y$. enterocolitica a genomic analysis between the two species could be performed. Alternatively, a TnSeq library of $Y$. enterocolitica could be used to infect mouse bone marrow neutrophils in a high throughput screen to determine what is triggering $L T B B_{4}$ release. The hits could be compared back to Y. pseudotuberculosis and Y. pestis to verify lack of expression or modification upon divergence from Y. enterocolitica.

$$
\text { A }
$$
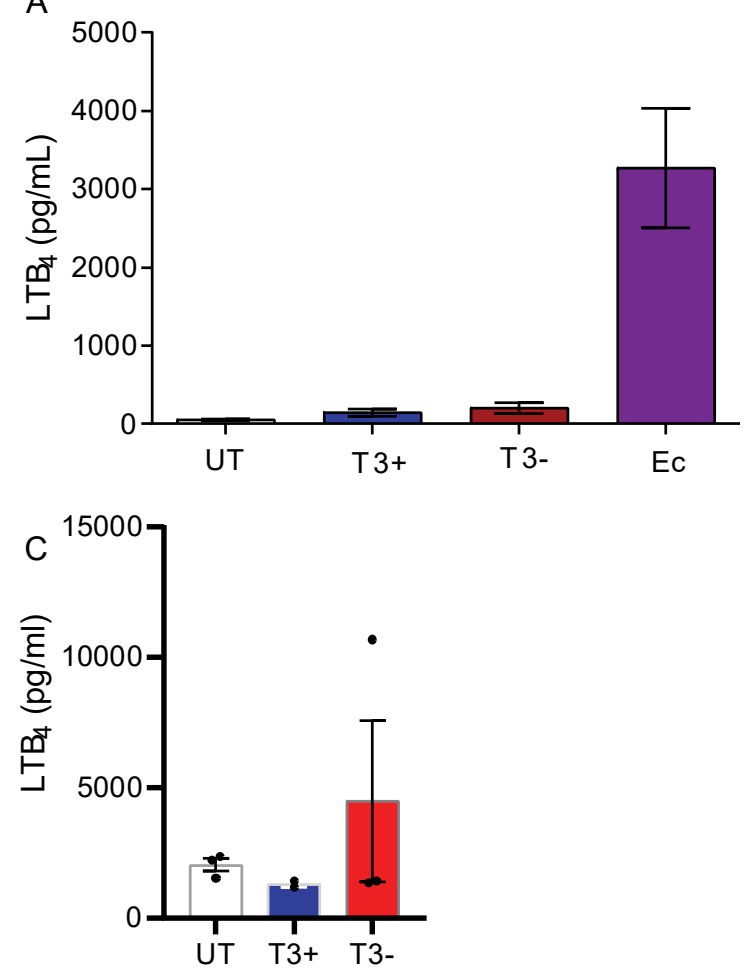

B

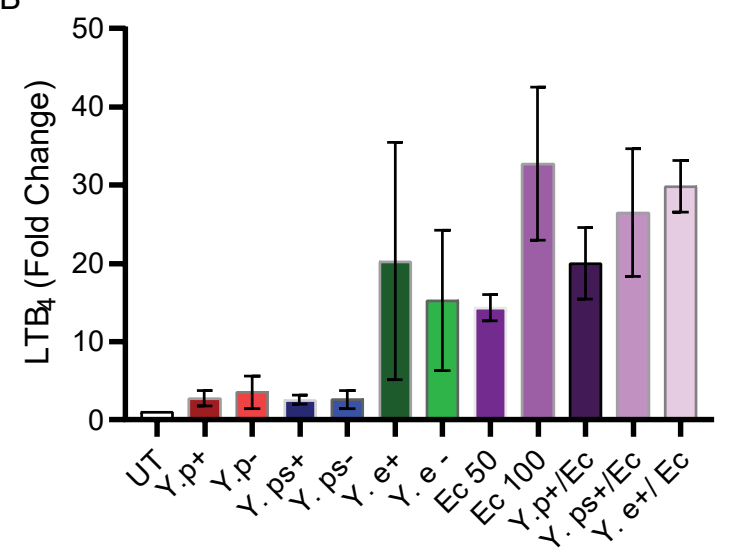

Figure 5-6: LTB 4 is not release from mouse phagocytes.

(A) Bone marrow derived mouse neutrophils $\left(1 \times 10^{6}\right)$ were infected with Y. pestis KIM1001 or CO92, with or without the pCD1 plasmid encoding the T3SS (T3+ or T3-, respectively), or E. coli (Ec) $\mathrm{MOI}=100$. Release of $\mathrm{LTB}_{4}$ was measured after 30 min of infection in supernatant. (B) Bone marrow derived mouse neutrophils $\left(1 \times 10^{6}\right)$ were infected with $Y$. pestis KIM1001 or C092, Y. pseudotuberculosis, or $Y$. enterocolitica with or without the pCD1 plasmid encoding the T3SS (Y.p+, Y.p-, Y.ps+, Y.ps-, Y.e+, or Y.e- , respectively), or E. coli (Ec) alone or mixed 1:1 with the indicated Yersinia strain; final $\mathrm{MOI}=100$, except for $E$. coli alone at $\mathrm{MOI}=50$ and release of $\mathrm{LTB}_{4}$ was measured $30 \mathrm{~min}$ after infection in the supernatant. Mean \pm SEM from 2-9 biologically 
independent experiments. (C) LTB 4 in BALF from C57BI6 mice were infected with fully virulent $Y$. pestis or lacking the pCD1 plasmid, T3+ and T3-, respectively. At $12 \mathrm{~h}$ post-infection, BALF was collected and $\mathrm{LTB}_{4}$ was concentrated using a $\mathrm{C} 4$ column. Mean \pm SEM from 3 mice for each sample.

\section{CONCLUSIONS}

Yersinia pestis has masterfully adapted to subverting destruction by the innate immune system by subverting destruction mediated by both macrophages and neutrophils. The ways in which Y. pestis subverts antimicrobial responses utilized by the respective cell types differs greatly. However, many questions still remain regarding the exact mechanisms used by $Y$. pestis to subvert macrophage and neutrophil antimicrobial responses. The work presented here, in addition to the observations I have made, aim to address those questions. I have presented data demonstrating that $Y$. pestis relies on eight host Rab GTPases to survive and replicate within macrophages, and that five Rabs are co-localized to the YCV. The mechanistic roles these Rab GTPases play during Y. pestis intracellular survival are areas for continued research.

Survival in macrophages provides $Y$. pestis the needed time to increase T3SS expression for survival outside of macrophages. Upon expression and exiting from macrophages, the T3SS inhibits further phagocytosis, and functions to combat antimicrobial defense mechanisms mounted by neutrophils. I demonstrated that $Y$. pestis inhibits release of all four neutrophil granule subtypes, and production of $\mathrm{LTB}_{4}$ through inhibition of the MAPK pathway. Furthermore, I have presented evidence that not only granule and $\mathrm{LTB}_{4}$ release are modified during $\mathrm{Y}$. pestis infection, but also that other neutrophil responses may be modified as well. I also found that the ways in which human and mouse cells respond to $Y$. pestis infection are not always similar. Understanding whether a phenotype recapitulates in a mouse model and why differences between human cells and mouse cells occur will allow us to better understand how $Y$. pestis causes disease in different hosts. Together, the information obtained from this work and future studies will enable us to understand how Y. pestis maintains an early non-inflammatory host environment, hopefully leading us to a way in which Y. pestis infection of humans can be eliminated. 


\section{REFERENCES}

1. Perry, R.D. and J.D. Fetherston, Yersinia pestis--etiologic agent of plague. Clin Microbiol Rev, 1997. 10(1): p. 35-66.

2. Chain, P.S., et al., Insights into the evolution of Yersinia pestis through whole-genome comparison with Yersinia pseudotuberculosis. Proc Natl Acad Sci U S A, 2004. 101(38): p. 13826-31.

3. Sebbane, F., et al., Kinetics of disease progression and host response in a rat model of bubonic plague. Am J Pathol, 2005. 166(5): p. 1427-39.

4. Comer, J.E., et al., Transcriptomic and innate immune responses to Yersinia pestis in the lymph node during bubonic plague. Infect Immun, 2010. 78(12): p. 5086-98.

5. Sun, Y., et al., Development of bioluminescent bioreporters for in vitro and in vivo tracking of Yersinia pestis. PLoS One, 2012. 7(10): p. e47123.

6. Agar, S.L., et al., Deletion of Braun lipoprotein gene (Ipp) and curing of plasmid PPCP1 dramatically alter the virulence of Yersinia pestis CO92 in a mouse model of pneumonic plague. Microbiology, 2009. 155(Pt 10): p. 3247-59.

7. Harbeck, M., et al., Yersinia pestis DNA from skeletal remains from the 6(th) century AD reveals insights into Justinianic Plague. PLoS pathogens, 2013. 9(5): p. e1003349e1003349.

8. Duncan, C.J. and S. Scott, What caused the Black Death? Postgrad Med J, 2005. 81(955): p. 315-20.

9. Haensch, S., et al., Distinct clones of Yersinia pestis caused the black death. PLoS Pathog, 2010. 6(10): p. e1001134.

10. Zietz, B.P. and H. Dunkelberg, The history of the plague and the research on the causative agent Yersinia pestis. Int J Hyg Environ Health, 2004. 207(2): p. 165-78.

11. Morelli, G., et al., Yersinia pestis genome sequencing identifies patterns of global phylogenetic diversity. Nat Genet, 2010. 42(12): p. 1140-3.

12. Schmid, B.V., et al., Climate-driven introduction of the Black Death and successive plague reintroductions into Europe. Proc Natl Acad Sci U S A, 2015. 112(10): p. 3020-5.

13. Sonne, O., Plague, rats, and ships The realisation of the infection routes of plague. Dan Medicinhist Arbog, 2016. 44: p. 101-133.

14. Griffin, K.A., et al., Detection of Yersinia pestis DNA in prairie dog-associated fleas by polymerase chain reaction assay of purified DNA. J Wildl Dis, 2010. 46(2): p. 636-43.

15. Salkeld, D.J., Vaccines for Conservation: Plague, Prairie Dogs \& Black-Footed Ferrets as a Case Study. Ecohealth, 2017. 14(3): p. 432-437.

16. Matchett, M.R., et al., Enzootic plague reduces black-footed ferret (Mustela nigripes) survival in Montana. Vector Borne Zoonotic Dis, 2010. 10(1): p. 27-35.

17. Schaffer, P.A., et al., Pneumonic Plague in a Dog and Widespread Potential Human Exposure in a Veterinary Hospital, United States. Emerg Infect Dis, 2019. 25(4): p. 800803.

18. Andrianaivoarimanana, V., et al., Trends of Human Plague, Madagascar, 1998-2016. Emerg Infect Dis, 2019. 25(2): p. 220-228. 
19. Miarinjara, A., et al., Field assessment of insecticide dusting and bait station treatment impact against rodent flea and house flea species in the Madagascar plague context. PLoS Negl Trop Dis, 2019. 13(8): p. e0007604.

20. Randremanana, R., et al., Epidemiological characteristics of an urban plague epidemic in Madagascar, August-November, 2017: an outbreak report. Lancet Infect Dis, 2019. 19(5): p. 537-545.

21. Rabaan, A.A., et al., The rise of pneumonic plague in Madagascar: current plague outbreak breaks usual seasonal mould. J Med Microbiol, 2019. 68(3): p. 292-302.

22. Wang, P., et al., Ten years of surveillance of the Yulong plague focus in China and the molecular typing and source tracing of the isolates. PLoS Negl Trop Dis, 2018. 12(3): p. e0006352.

23. Shabbir, M., et al., Spatial analysis and identification of high risk plague regions in Pakistan based on associated rodent species distribution. J Infect Dev Ctries, 2016. 10(7): p. 687-93.

24. Carus, W.S., Biological Warfare in the 17th Century. Emerging infectious diseases, 2016. 22(9): p. 1663-1664.

25. Riedel, S., Plague: from natural disease to bioterrorism. Proceedings (Baylor University. Medical Center), 2005. 18(2): p. 116-124.

26. Harris, S., Japanese biological warfare research on humans: a case study of microbiology and ethics. Ann N Y Acad Sci, 1992. 666: p. 21-52.

27. Davis, K.M., All Yersinia Are Not Created Equal: Phenotypic Adaptation to Distinct Niches Within Mammalian Tissues. Frontiers in Cellular and Infection Microbiology, 2018. 8: p. 261.

28. Hubbert, W.T., et al., Yersinia pseudotuberculosis infection in the United States. Speticema, appendicitis, and mesenteric lymphadenitis. Am J Trop Med Hyg, 1971. 20(5): p. 679-84.

29. Paff, J.R., D.A. Triplett, and T.N. Saari, Clinical and laboratory aspects of Yersinia pseudotuberculosis infections, with a report of two cases. Am J Clin Pathol, 1976. 66(1): p. 101-10.

30. Balada-Llasat, J.-M. and J. Mecsas, Yersinia has a tropism for $B$ and $T$ cell zones of lymph nodes that is independent of the type III secretion system. PLoS pathogens, 2006. 2(9): p. e86-e86.

31. El-Maraghi, N.R. and N.S. Mair, The histopathology of enteric infection with Yersinia pseudotuberculosis. Am J Clin Pathol, 1979. 71(6): p. 631-9.

32. Lian, C.J., W.S. Hwang, and C.H. Pai, Plasmid-mediated resistance to phagocytosis in Yersinia enterocolitica. Infection and immunity, 1987. 55(5): p. 1176-1183.

33. Bohn, E., et al., Bacterial adhesion and host cell factors leading to effector protein injection by type III secretion system. Int J Med Microbiol, 2019. 309(5): p. 344-350.

34. Anderson, D.M., et al., Pneumonic plague pathogenesis and immunity in Brown Norway rats. Am J Pathol, 2009. 174(3): p. 910-21.

35. Bergsbaken, T. and B.T. Cookson, Innate immune response during Yersinia infection: critical modulation of cell death mechanisms through phagocyte activation. Journal of leukocyte biology, 2009. 86(5): p. 1153-1158.

36. Bosio, C.F., et al., Kinetics of innate immune response to Yersinia pestis after intradermal infection in a mouse model. Infect Immun, 2012. 80(11): p. 4034-45.

37. Lathem, W.W., et al., Progression of primary pneumonic plague: a mouse model of infection, pathology, and bacterial transcriptional activity. Proceedings of the National Academy of Sciences of the United States of America, 2005. 102(49): p. 17786-17791. 
38. Tamassia, N., et al., Cytokine production by human neutrophils: Revisiting the "dark side of the moon". Eur J Clin Invest, 2018. 48 Suppl 2: p. e12952.

39. Spinner, J.L., et al., Yersinia pestis survival and replication within human neutrophil phagosomes and uptake of infected neutrophils by macrophages. J Leukoc Biol, 2014. 95(3): p. 389-98.

40. Shannon, J.G., et al., Yersinia pestis subverts the dermal neutrophil response in a mouse model of bubonic plague. MBio, 2013. 4(5): p. e00170-13.

41. Shannon, J.G., C.F. Bosio, and B.J. Hinnebusch, Dermal neutrophil, macrophage and dendritic cell responses to Yersinia pestis transmitted by fleas. PLoS pathogens, 2015. 11(3): p. e1004734-e1004734.

42. Marketon, M.M., et al., Plague bacteria target immune cells during infection. Science, 2005. 309(5741): p. 1739-41.

43. Merritt, P.M., et al., Yersinia pestis targets neutrophils via complement receptor 3. Cellular Microbiology, 2015. 17(5): p. 666-687.

44. Pha, K. and L. Navarro, Yersinia type III effectors perturb host innate immune responses. World J Biol Chem, 2016. 7(1): p. 1-13.

45. Bartra, S.S., et al., Resistance of Yersinia pestis to complement-dependent killing is mediated by the Ail outer membrane protein. Infect Immun, 2008. 76(2): p. 612-22.

46. Bland, D.M. and D.M. Anderson, Imaging early pathogenesis of bubonic plague: are neutrophils commandeered for lymphatic transport of bacteria? MBio, 2013. 4(6): p. e00837-13.

47. Blander, J.M. and R. Medzhitov, Regulation of phagosome maturation by signals from toll-like receptors. Science, 2004. 304(5673): p. 1014-8.

48. Montminy, S.W., et al., Virulence factors of Yersinia pestis are overcome by a strong lipopolysaccharide response. Nat Immunol, 2006. 7(10): p. 1066-73.

49. Rebeil, R., et al., Variation in lipid A structure in the pathogenic yersiniae. Mol Microbiol, 2004. 52(5): p. 1363-73.

50. Hajjar, A.M., et al., Humanized TLR4/MD-2 mice reveal LPS recognition differentially impacts susceptibility to Yersinia pestis and Salmonella enterica. PLoS pathogens, 2012. 8(10): p. e1002963-e1002963.

51. Matsuura, M., et al., Immunomodulatory effects of Yersinia pestis lipopolysaccharides on human macrophages. Clin Vaccine Immunol, 2010. 17(1): p. 49-55.

52. Yang, K., et al., Yersinia pestis Interacts With SIGNR1 (CD209b) for Promoting Host Dissemination and Infection. Frontiers in immunology, 2019. 10: p. 96-96.

53. Hinnebusch, B.J., et al., Role of the Yersinia pestis Ail protein in preventing a protective polymorphonuclear leukocyte response during bubonic plague. Infect Immun, 2011. 79(12): p. 4984-9.

54. Zhang, Y., et al., Invasiveness of the Yersinia pestis ail protein contributes to host dissemination in pneumonic and oral plague. Microb Pathog, 2020. 141: p. 103993.

55. Paczosa, M.K., et al., Yersinia pseudotuberculosis uses Ail and YadA to circumvent neutrophils by directing Yop translocation during lung infection. Cell Microbiol, 2014. 16(2): p. 247-68.

56. Pierson, D.E. and S. Falkow, The ail gene of Yersinia enterocolitica has a role in the ability of the organism to survive serum killing. Infection and immunity, 1993. 61(5): p. 18461852.

57. Kolodziejek, A.M., et al., Outer membrane protein X (Ail) contributes to Yersinia pestis virulence in pneumonic plague and its activity is dependent on the lipopolysaccharide core length. Infection and immunity, 2010. 78(12): p. 5233-5243. 
58. Cowan, C., et al., Invasion of epithelial cells by Yersinia pestis: evidence for a Y. pestisspecific invasin. Infect Immun, 2000. 68(8): p. 4523-30.

59. Eddy, J.L., et al., Proteolysis of plasminogen activator inhibitor-1 by Yersinia pestis remodulates the host environment to promote virulence. J Thromb Haemost, 2016. 14(9): p. 1833-43.

60. Banerjee, S.K., et al., Modeling Pneumonic Plague in Human Precision-Cut Lung Slices Highlights a Role for the Plasminogen Activator Protease in Facilitating Type 3 Secretion. Infect Immun, 2019. 87(8).

61. Banerjee, S.K., S.D. Crane, and R.D. Pechous, A dual role for the Plasminogen activator protease during the pre-inflammatory phase of primary pneumonic plague. J Infect Dis, 2020.

62. Du, Y., R. Rosqvist, and A. Forsberg, Role of fraction 1 antigen of Yersinia pestis in inhibition of phagocytosis. Infect Immun, 2002. 70(3): p. 1453-60.

63. Sha, J., et al., Characterization of an F1 deletion mutant of Yersinia pestis CO92, pathogenic role of F1 antigen in bubonic and pneumonic plague, and evaluation of sensitivity and specificity of F1 antigen capture-based dipsticks. Journal of clinical microbiology, 2011. 49(5): p. 1708-1715.

64. Hatkoff, M., et al., Roles of chaperone/usher pathways of Yersinia pestis in a murine model of plague and adhesion to host cells. Infect Immun, 2012. 80(10): p. 3490-500.

65. Runco, L.M., et al., Biogenesis of the fraction 1 capsule and analysis of the ultrastructure of Yersinia pestis. J Bacteriol, 2008. 190(9): p. 3381-5.

66. Galvan, E.M., M.A. Lasaro, and D.M. Schifferli, Capsular antigen fraction 1 and Pla modulate the susceptibility of Yersinia pestis to pulmonary antimicrobial peptides such as cathelicidin. Infect Immun, 2008. 76(4): p. 1456-64.

67. Green, E.R. and J. Mecsas, Bacterial Secretion Systems: An Overview. Microbiology spectrum, 2016. 4(1): p. 10.1128/microbiolspec.VMBF-0012-2015.

68. Puhar, A. and P.J. Sansonetti, Type III secretion system. Curr Biol, 2014. 24(17): p. R78491.

69. Bliska, J.B., et al., Modulation of innate immune responses by Yersinia type III secretion system translocators and effectors. Cell Microbiol, 2013. 15(10): p. 1622-31.

70. Chung, L.K. and J.B. Bliska, Yersinia versus host immunity: how a pathogen evades or triggers a protective response. Curr Opin Microbiol, 2016. 29: p. 56-62.

71. Mukherjee, S., et al., Yersinia YopJ acetylates and inhibits kinase activation by blocking phosphorylation. Science, 2006. 312(5777): p. 1211-4.

72. Andor, A., et al., YopE of Yersinia, a GAP for Rho GTPases, selectively modulates Racdependent actin structures in endothelial cells. Cell Microbiol, 2001. 3(5): p. 301-10.

73. Black, D.S. and J.B. Bliska, Identification of p130Cas as a substrate of Yersinia YopH (Yop51), a bacterial protein tyrosine phosphatase that translocates into mammalian cells and targets focal adhesions. Embo j, 1997. 16(10): p. 2730-44.

74. Black, D.S. and J.B. Bliska, The RhoGAP activity of the Yersinia pseudotuberculosis cytotoxin YopE is required for antiphagocytic function and virulence. Mol Microbiol, 2000. 37(3): p. 515-27.

75. Bliska, J.B., Yop effectors of Yersinia spp. and actin rearrangements. Trends Microbiol, 2000. 8(5): p. 205-8.

76. Iriarte, M. and G.R. Cornelis, YopT, a new Yersinia Yop effector protein, affects the cytoskeleton of host cells. Mol Microbiol, 1998. 29(3): p. 915-29.

77. Barz, C., et al., The Yersinia Ser/Thr protein kinase YpkA/YopO directly interacts with the small GTPases RhoA and Rac-1. FEBS Lett, 2000. 482(1-2): p. 139-43. 
78. Fueller, F., et al., Endoproteolytic processing of RhoA by Rce 1 is required for the cleavage of RhoA by Yersinia enterocolitica outer protein T. Infect Immun, 2006. 74(3): p. 1712-7.

79. Sorg, I., et al., Recombinant Yersinia YopT leads to uncoupling of RhoA-effector interaction. Infect Immun, 2001. 69(12): p. 7535-43.

80. Zumbihl, R., et al., The cytotoxin YopT of Yersinia enterocolitica induces modification and cellular redistribution of the small GTP-binding protein RhoA. J Biol Chem, 1999. 274(41): p. 29289-93.

81. Andersson, K., et al., Yersinia pseudotuberculosis-induced calcium signaling in neutrophils is blocked by the virulence effector YopH. Infect Immun, 1999. 67(5): p. 2567-74.

82. Black, D.S., et al., Identification of an amino-terminal substrate-binding domain in the Yersinia tyrosine phosphatase that is required for efficient recognition of focal adhesion targets. Mol Microbiol, 1998. 29(5): p. 1263-74.

83. Cantwell, A.M., S.S. Bubeck, and P.H. Dube, YopH inhibits early pro-inflammatory cytokine responses during plague pneumonia. BMC Immunol, 2010. 11: p. 29.

84. Sauvonnet, N., et al., YopH prevents monocyte chemoattractant protein 1 expression in macrophages and T-cell proliferation through inactivation of the phosphatidylinositol 3kinase pathway. Mol Microbiol, 2002. 45(3): p. 805-15.

85. Arifuzzaman, M., et al., Necroptosis of infiltrated macrophages drives Yersinia pestis dispersal within buboes. JCI Insight, 2018. 3(18).

86. Lemaitre, N., et al., Yersinia pestis YopJ suppresses tumor necrosis factor alpha induction and contributes to apoptosis of immune cells in the lymph node but is not required for virulence in a rat model of bubonic plague. Infect Immun, 2006. 74(9): p. 5126-31.

87. Mukherjee, S. and K. Orth, In vitro signaling by MAPK and NFkappaB pathways inhibited by Yersinia YopJ. Methods Enzymol, 2008. 438: p. 343-53.

88. Zhou, H., et al., Yersinia virulence factor YopJ acts as a deubiquitinase to inhibit NFkappa B activation. J Exp Med, 2005. 202(10): p. 1327-32.

89. Menon, M.B., et al., p38(MAPK)/MK2-dependent phosphorylation controls cytotoxic RIPK1 signalling in inflammation and infection. Nat Cell Biol, 2017. 19(10): p. 1248-1259.

90. Peterson, L.W., et al., RIPK1-dependent apoptosis bypasses pathogen blockade of innate signaling to promote immune defense. J Exp Med, 2017. 214(11): p. 3171-3182.

91. Chung, L.K., et al., The Yersinia Virulence Factor YopM Hijacks Host Kinases to Inhibit Type III Effector-Triggered Activation of the Pyrin Inflammasome. Cell Host Microbe, 2016. 20(3): p. 296-306.

92. Dewoody, R., P. Merritt, and M. Marketon, Regulation of the Yersinia type III secretion system: traffic control. Frontiers in Cellular and Infection Microbiology, 2013. 3(4).

93. Peters, K.N. and D.M. Anderson, Modulation of host cell death pathways by Yersinia species and the type III effector YopK. Adv Exp Med Biol, 2012. 954: p. 229-36.

94. Tan, Y., et al., Yersinia pestis YopK Inhibits Bacterial Adhesion to Host Cells by Binding to the Extracellular Matrix Adaptor Protein Matrilin-2. Infect Immun, 2017. 85(8).

95. Thorslund, S.E., et al., Role of YopK in Yersinia pseudotuberculosis resistance against polymorphonuclear leukocyte defense. Infect Immun, 2013. 81(1): p. 11-22.

96. Zwack, E.E., et al., Guanylate Binding Proteins Regulate Inflammasome Activation in Response to Hyperinjected Yersinia Translocon Components. Infect Immun, 2017. 85(10).

97. Ratner, D., et al., The Yersinia pestis Effector YopM Inhibits Pyrin Inflammasome Activation. PLoS Pathog, 2016. 12(12): p. e1006035.

98. Uittenbogaard, A.M., et al., Toward a molecular pathogenic pathway for Yersinia pestis YopM. Front Cell Infect Microbiol, 2012. 2: p. 155. 
99. Hentschke, M., et al., Yersinia virulence factor YopM induces sustained RSK activation by interfering with dephosphorylation. PLoS One, 2010. 5(10).

100. Brodsky, I.E., et al., A Yersinia effector protein promotes virulence by preventing inflammasome recognition of the type III secretion system. Cell Host Microbe, 2010. 7(5): p. 376-87.

101. Hooker-Romero, D., et al., Iron availability and oxygen tension regulate the Yersinia YSC type III secretion system to enable disseminated infection. PLoS pathogens, 2019. 15(12): p. e1008001-e1008001.

102. Plano, G.V. and K. Schesser, The Yersinia pestis type III secretion system: expression, assembly and role in the evasion of host defenses. Immunol Res, 2013. 57(1-3): p. 23745.

103. Pollack, C., S.C. Straley, and M.S. Klempner, Probing the phagolysosomal environment of human macrophages with a Ca2+-responsive operon fusion in Yersinia pestis. Nature, 1986. 322(6082): p. 834-6.

104. Barve, S.S. and S.C. Straley, IcrR, a low-Ca2(+)-response locus with dual Ca2(+)dependent functions in Yersinia pestis. J Bacteriol, 1990. 172(8): p. 4661-71.

105. Fields, K.A., G.V. Plano, and S.C. Straley, A low-Ca2+ response (LCR) secretion (ysc) locus lies within the ICrB region of the LCR plasmid in Yersinia pestis. J Bacteriol, 1994. 176(3): p. 569-79.

106. Charnetzky, W.T. and W.W. Shuford, Survival and growth of Yersinia pestis within macrophages and an effect of the loss of the 47-megadalton plasmid on growth in macrophages. Infect Immun, 1985. 47(1): p. 234-41.

107. Straley, S.C. and P.A. Harmon, Growth in mouse peritoneal macrophages of Yersinia pestis lacking established virulence determinants. Infect Immun, 1984. 45(3): p. 649-54.

108. Straley, S.C. and P.A. Harmon, Yersinia pestis grows within phagolysosomes in mouse peritoneal macrophages. Infect Immun, 1984. 45(3): p. 655-9.

109. Pujol, C. and J.B. Bliska, Turning Yersinia pathogenesis outside in: subversion of macrophage function by intracellular yersiniae. Clin Immunol, 2005. 114(3): p. 216-26.

110. St John, A.L., et al., S1P-Dependent trafficking of intracellular Yersinia pestis through lymph nodes establishes Buboes and systemic infection. Immunity, 2014. 41(3): p. 440450.

111. Pachulec, E., et al., Enhanced Macrophage M1 Polarization and Resistance to Apoptosis Enable Resistance to Plague. J Infect Dis, 2017. 216(6): p. 761-770.

112. Blanchet, C., et al., Mus spretus SEG/Pas mice resist virulent Yersinia pestis, under multigenic control. Genes \& Immunity, 2011. 12(1): p. 23-30.

113. Roberts, E.A., et al., Higher order Rab programming in phagolysosome biogenesis. J Cell Biol, 2006. 174(7): p. 923-9.

114. Schwartz, S.L., et al., Rab GTPases at a glance. Journal of Cell Science, 2008. 121(2): p. 246-246.

115. Gillingham, A.K., et al., Toward a comprehensive map of the effectors of rab GTPases. Dev Cell, 2014. 31(3): p. 358-73.

116. Andersson, J.A., et al., Identification of New Virulence Factors and Vaccine Candidates for Yersinia pestis. Front Cell Infect Microbiol, 2017. 7: p. 448.

117. Pujol, C., et al., Yersinia pestis can reside in autophagosomes and avoid xenophagy in murine macrophages by preventing vacuole acidification. Infect Immun, 2009. 77(6): p. 2251-61.

118. Connor, M.G., et al., Yersinia pestis Requires Host Rab1b for Survival in Macrophages. PLoS Pathog, 2015. 11(10): p. e1005241. 
119. Connor, M.G., et al., Yersinia pestis Targets the Host Endosome Recycling Pathway during the Biogenesis of the Yersinia-Containing Vacuole To Avoid Killing by Macrophages. MBio, 2018. 9(1).

120. Ligeon, L.A., et al., Role of VAMP3 and VAMP7 in the commitment of Yersinia pseudotuberculosis to LC3-associated pathways involving single- or double-membrane vacuoles. Autophagy, 2014. 10(9): p. 1588-602.

121. Moreau, K., et al., Autophagosomes can support Yersinia pseudotuberculosis replication in macrophages. Cell Microbiol, 2010. 12(8): p. 1108-23.

122. VanCleave, T.T., et al., Impact of Gentamicin Concentration and Exposure Time on Intracellular Yersinia pestis. Front Cell Infect Microbiol, 2017. 7: p. 505.

123. Grabenstein, J.P., et al., Characterization of phagosome trafficking and identification of PhoP-regulated genes important for survival of Yersinia pestis in macrophages. Infect Immun, 2006. 74(7): p. 3727-41.

124. Oyston, P.C., et al., The response regulator PhoP is important for survival under conditions of macrophage-induced stress and virulence in Yersinia pestis. Infect Immun, 2000. 68(6): p. 3419-25.

125. Fukuto, H.S., et al., Global gene expression profiling of Yersinia pestis replicating inside macrophages reveals the roles of a putative stress-induced operon in regulating type III secretion and intracellular cell division. Infect Immun, 2010. 78(9): p. 3700-15.

126. Pujol, C., et al., Replication of Yersinia pestis in interferon gamma-activated macrophages requires ripA, a gene encoded in the pigmentation locus. Proc Natl Acad Sci U S A, 2005. 102(36): p. 12909-14.

127. Lamb, C.A., A. Longatti, and S.A. Tooze, Rabs and GAPs in starvation-induced autophagy. Small GTPases, 2016. 7(4): p. 265-269.

128. Parzych, K.R. and D.J. Klionsky, An overview of autophagy: morphology, mechanism, and regulation. Antioxid Redox Signal, 2014. 20(3): p. 460-73.

129. Nozawa, T., et al., The STX6-VTI1B-VAMP3 complex facilitates xenophagy by regulating the fusion between recycling endosomes and autophagosomes. Autophagy, 2017. 13(1): p. 57-69.

130. Longatti, A., et al., TBC1D14 regulates autophagosome formation via Rab11- and ULK1positive recycling endosomes. J Cell Biol, 2012. 197(5): p. 659-75.

131. Koyama-Honda, I., K. Tsuboyama, and N. Mizushima, ATG conjugation-dependent degradation of the inner autophagosomal membrane is a key step for autophagosome maturation. Autophagy, 2017. 13(7): p. 1252-1253.

132. Itakura, E., C. Kishi-Itakura, and N. Mizushima, The Hairpin-type Tail-Anchored SNARE Syntaxin 17 Targets to Autophagosomes for Fusion with Endosomes/Lysosomes. Cell, 2012. 151(6): p. 1256-1269.

133. Campbell-Valois, F.X., et al., Escape of Actively Secreting Shigella flexneri from ATG8/LC3-Positive Vacuoles Formed during Cell-To-Cell Spread Is Facilitated by IcsB and VirA. MBio, 2015. 6(3).

134. Dong, N., et al., Structurally distinct bacterial TBC-like GAPs link Arf GTPase to Rab1 inactivation to counteract host defenses. Cell, 2012. 150(5): p. 1029-41.

135. Romano, P.S., et al., The autophagic pathway is actively modulated by phase II Coxiella burnetii to efficiently replicate in the host cell. Cell Microbiol, 2007. 9(4): p. 891-909.

136. Schulze-Luehrmann, J., et al., LAMP proteins account for the maturation delay during the establishment of the Coxiella burnetii-containing vacuole. Cell Microbiol, 2015.

137. Winchell, C.G., et al., Coxiella burnetii Subverts p62/Sequestosome 1 and Activates Nrf2 Signaling in Human Macrophages. Infect Immun, 2018. 86(5). 
138. Niu, H., M. Yamaguchi, and Y. Rikihisa, Subversion of cellular autophagy by Anaplasma phagocytophilum. Cell Microbiol, 2008. 10(3): p. 593-605.

139. Agaisse, H., et al., Genome-wide RNAi screen for host factors required for intracellular bacterial infection. Science, 2005. 309(5738): p. 1248-51.

140. Casanova, A., et al., A Role for the VPS Retromer in Brucella Intracellular Replication Revealed by Genomewide siRNA Screening. mSphere, 2019. 4(3).

141. Rother, M., et al., Combined Human Genome-wide RNAi and Metabolite Analyses Identify IMPDH as a Host-Directed Target against Chlamydia Infection. Cell Host Microbe, 2018. 23(5): p. 661-671.e8.

142. Misselwitz, B., et al., RNAi screen of Salmonella invasion shows role of COPI in membrane targeting of cholesterol and Cdc42. Mol Syst Biol, 2011. 7: p. 474.

143. Thornbrough, J.M., et al., Human Genome-Wide RNAi Screen for Host Factors That Facilitate Salmonella Invasion Reveals a Role for Potassium Secretion in Promoting Internalization. PLoS One, 2016. 11(11): p. e0166916.

144. Thornbrough, J.M., et al., Human genome-wide RNAi screen for host factors that modulate intracellular Salmonella growth. PLoS One, 2012. 7(6): p. e38097.

145. Ye, Z., et al., Gr1+ cells control growth of YopM-negative Yersinia pestis during systemic plague. Infect Immun, 2009. 77(9): p. 3791-806.

146. Vagima, Y., et al., Circumventing Y. pestis Virulence by Early Recruitment of Neutrophils to the Lungs during Pneumonic Plague. PLoS Pathog, 2015. 11(5): p. e1004893.

147. Dudte, S.C., B.J. Hinnebusch, and J.G. Shannon, Characterization of Yersinia pestis Interactions with Human Neutrophils In vitro. Front Cell Infect Microbiol, 2017. 7: p. 358.

148. Ley, K., et al., Neutrophils: New insights and open questions. Sci Immunol, 2018. 3(30).

149. Mayadas, T.N., X. Cullere, and C.A. Lowell, The multifaceted functions of neutrophils. Annual review of pathology, 2014. 9: p. 181-218.

150. Aga, E., et al., Type-1 interferons prolong the lifespan of neutrophils by interfering with members of the apoptotic cascade. Cytokine, 2018. 112: p. 21-26.

151. Liew, P.X. and P. Kubes, The Neutrophil's Role During Health and Disease. Physiol Rev, 2019. 99(2): p. 1223-1248.

152. Kienle, K. and T. Lämmermann, Neutrophil swarming: an essential process of the neutrophil tissue response. Immunological Reviews, 2016. 273(1): p. 76-93.

153. Uriarte, S.M., et al., Granule exocytosis contributes to priming and activation of the human neutrophil respiratory burst. J Immunol, 2011. 187(1): p. 391-400.

154. Abdel-Latif, D., et al., Rac2 is critical for neutrophil primary granule exocytosis. Blood, 2004. 104(3): p. 832-9.

155. Lacy, P., Mechanisms of degranulation in neutrophils. Allergy, asthma, and clinical immunology : official journal of the Canadian Society of Allergy and Clinical Immunology, 2006. 2(3): p. 98-108.

156. Thanabalasuriar, A., et al., Neutrophil Extracellular Traps Confine Pseudomonas aeruginosa Ocular Biofilms and Restrict Brain Invasion. Cell Host Microbe, 2019. 25(4): p. 526-536.e4.

157. Urban, C.F., et al., Neutrophil extracellular traps contain calprotectin, a cytosolic protein complex involved in host defense against Candida albicans. PLoS Pathog, 2009. 5(10): p. e1000639.

158. Nordenfelt, P. and H. Tapper, Phagosome dynamics during phagocytosis by neutrophils. J Leukoc Biol, 2011. 90(2): p. 271-84. 
159. Ward, R.A., M. Nakamura, and K.R. McLeish, Priming of the neutrophil respiratory burst involves $p 38$ mitogen-activated protein kinase-dependent exocytosis of flavocytochrome b558-containing granules. J Biol Chem, 2000. 275(47): p. 36713-9.

160. Condliffe, A.M., et al., RhoG regulates the neutrophil NADPH oxidase. J Immunol, 2006. 176(9): p. 5314-20.

161. Creed, T.M., et al., Endocytosis is required for exocytosis and priming of respiratory burst activity in human neutrophils. Inflamm Res, 2017. 66(10): p. 891-899.

162. Downey, G.P., et al., Importance of MEK in neutrophil microbicidal responsiveness. J Immunol, 1998. 160(1): p. 434-43.

163. Batra, S., et al., Intrapulmonary administration of leukotriene B(4) augments neutrophil accumulation and responses in the lung to Klebsiella infection in CXCL1 knockout mice. J Immunol, 2012. 188(7): p. 3458-68.

164. El Jamali, A., A.J. Valente, and R.A. Clark, Regulation of phagocyte NADPH oxidase by hydrogen peroxide through a $\mathrm{Ca}(2+) / c-A b l$ signaling pathway. Free Radic Biol Med, 2010. 48(6): p. 798-810.

165. Nauseef, W.M., How human neutrophils kill and degrade microbes: an integrated view. Immunol Rev, 2007. 219: p. 88-102.

166. Spinner, J.L., J.A. Cundiff, and S.D. Kobayashi, Yersinia pestis type III secretion systemdependent inhibition of human polymorphonuclear leukocyte function. Infect Immun, 2008. 76(8): p. 3754-60.

167. Cowland, J.B. and N. Borregaard, Granulopoiesis and granules of human neutrophils. Immunol Rev, 2016. 273(1): p. 11-28.

168. Faurschou, M. and N. Borregaard, Neutrophil granules and secretory vesicles in inflammation. Microbes Infect, 2003. 5(14): p. 1317-27.

169. Ramadass, M. and S.D. Catz, Molecular mechanisms regulating secretory organelles and endosomes in neutrophils and their implications for inflammation. Immunol Rev, 2016. 273(1): p. 249-65.

170. Jog, N.R., et al., The actin cytoskeleton regulates exocytosis of all neutrophil granule subsets. Am J Physiol Cell Physiol, 2007. 292(5): p. C1690-700.

171. Johnson, J.L., et al., Vesicular trafficking through cortical actin during exocytosis is regulated by the Rab27a effector JFC1/SIp1 and the RhoA-GTPase-activating protein Gem-interacting protein. Molecular biology of the cell, 2012. 23(10): p. 1902-1916.

172. Mitchell, T., et al., Primary granule exocytosis in human neutrophils is regulated by Racdependent actin remodeling. American journal of physiology. Cell physiology, 2008. 295(5): p. C1354-C1365.

173. Sheshachalam, A., et al., Granule protein processing and regulated secretion in neutrophils. Front Immunol, 2014. 5: p. 448.

174. Papayannopoulos, V., et al., Neutrophil elastase and myeloperoxidase regulate the formation of neutrophil extracellular traps. J Cell Biol, 2010. 191(3): p. 677-91.

175. Tatsiy, O. and P.P. McDonald, Physiological Stimuli Induce PAD4-Dependent, ROSIndependent NETosis, With Early and Late Events Controlled by Discrete Signaling Pathways. Front Immunol, 2018. 9: p. 2036.

176. Hawez, A., et al., MiR-155 Regulates PAD4-Dependent Formation of Neutrophil Extracellular Traps. Front Immunol, 2019. 10: p. 2462.

177. Yipp, B.G. and P. Kubes, NETosis: how vital is it? Blood, 2013. 122(16): p. 2784-94.

178. Gray, R.D., et al., Activation of conventional protein kinase $C(P K C)$ is critical in the generation of human neutrophil extracellular traps. Journal of inflammation (London, England), 2013. 10(1): p. 12-12. 
179. Stoiber, W., et al., The Role of Reactive Oxygen Species (ROS) in the Formation of Extracellular Traps (ETs) in Humans. Biomolecules, 2015. 5(2): p. 702-23.

180. Lammermann, T., et al., Neutrophil swarms require LTB4 and integrins at sites of cell death in vivo. Nature, 2013. 498(7454): p. 371-5.

181. Peters-Golden, M., et al., Leukotrienes: underappreciated mediators of innate immune responses. J Immunol, 2005. 174(2): p. 589-94.

182. Bozza, P.T., et al., Lipid body function in eicosanoid synthesis: an update. Prostaglandins Leukot Essent Fatty Acids, 2011. 85(5): p. 205-13.

183. Dvorak, A.M., et al., Lipid bodies: cytoplasmic organelles important to arachidonate metabolism in macrophages and mast cells. J Immunol, 1983. 131(6): p. 2965-76.

184. Doerfler, M.E., et al., Bacterial lipopolysaccharide primes human neutrophils for enhanced release of arachidonic acid and causes phosphorylation of an 85-kD cytosolic phospholipase A2. The Journal of clinical investigation, 1994. 93(4): p. 1583-1591.

185. Hafner, A.K., et al., Characterization of the interaction of human 5-lipoxygenase with its activating protein FLAP. Biochim Biophys Acta, 2015. 1851(11): p. 1465-72.

186. Basavarajappa, D., et al., Roles of coactosin-like protein (CLP) and 5-lipoxygenaseactivating protein (FLAP) in cellular leukotriene biosynthesis. Proc Natl Acad Sci U S A, 2014. 111(31): p. 11371-6.

187. Kim, C.E., et al., Acrolein increases 5-lipoxygenase expression in murine macrophages through activation of ERK pathway. Toxicol Appl Pharmacol, 2010. 245(1): p. 76-82.

188. Werz, O., et al., 5-lipoxygenase is phosphorylated by p38 kinase-dependent MAPKAP kinases. Proceedings of the National Academy of Sciences of the United States of America, 2000. 97(10): p. 5261-5266.

189. Radmark, O. and B. Samuelsson, 5-Lipoxygenase: mechanisms of regulation. J Lipid Res, 2009. 50 Suppl: p. S40-5.

190. Radmark, O. and B. Samuelsson, Regulation of the activity of 5-lipoxygenase, a key enzyme in leukotriene biosynthesis. Biochem Biophys Res Commun, 2010. 396(1): p. 105-10.

191. Radmark, O., et al., 5-Lipoxygenase: regulation of expression and enzyme activity. Trends Biochem Sci, 2007. 32(7): p. 332-41.

192. Fischer, L., et al., Inhibitors of actin polymerisation stimulate arachidonic acid release and 5-lipoxygenase activation by upregulation of Ca2+ mobilisation in polymorphonuclear leukocytes involving Src family kinases. Biochimica et Biophysica Acta (BBA) - Molecular and Cell Biology of Lipids, 2005. 1736(2): p. 109-119.

193. Esser, J., et al., Exosomes from human macrophages and dendritic cells contain enzymes for leukotriene biosynthesis and promote granulocyte migration. J Allergy Clin Immunol, 2010. 126(5): p. 1032-40, 1040.e1-4.

194. Majumdar, R., A. Tavakoli Tameh, and C.A. Parent, Exosomes Mediate LTB4 Release during Neutrophil Chemotaxis. PLoS Biol, 2016. 14(1): p. e1002336.

195. Lam, B.K., et al., The mechanism of leukotriene B4 export from human polymorphonuclear leukocytes. J Biol Chem, 1990. 265(23): p. 13438-41.

196. Mancuso, P., P. Nana-Sinkam, and M. Peters-Golden, Leukotriene B4 augments neutrophil phagocytosis of Klebsiella pneumoniae. Infect Immun, 2001. 69(4): p. 2011-6.

197. Pires-Lapa, M.A., et al., Leukotriene B4 modulation of murine dendritic cells affects adaptive immunity. Prostaglandins Other Lipid Mediat, 2019. 141: p. 34-39.

198. Serezani, C.H., et al., Leukotrienes enhance the bactericidal activity of alveolar macrophages against Klebsiella pneumoniae through the activation of NADPH oxidase. Blood, 2005. 106(3): p. 1067-75. 
199. Soares, E.M., et al., Leukotriene B4 enhances innate immune defense against the puerperal sepsis agent Streptococcus pyogenes. J Immunol, 2013. 190(4): p. 1614-22.

200. Brandt, S.L. and C.H. Serezani, Too much of a good thing: How modulating LTB4 actions restore host defense in homeostasis or disease. Semin Immunol, 2017. 33: p. 37-43.

201. Coffey, M.J., S.M. Phare, and M. Peters-Golden, Role of leukotrienes in killing of Mycobacterium bovis by neutrophils. Prostaglandins, Leukotrienes and Essential Fatty Acids, 2004. 71(3): p. 185-190.

202. Prado, M.K.B., et al., Leukotriene B4 is essential for lung host defence and alphadefensin-1 production during Achromobacter xylosoxidans infection. Sci Rep, 2017. 7(1): p. 17658.

203. Bailie, M.B., et al., Leukotriene-deficient mice manifest enhanced lethality from Klebsiella pneumonia in association with decreased alveolar macrophage phagocytic and bactericidal activities. J Immunol, 1996. 157(12): p. 5221-4.

204. Del Prete, A., et al., Regulation of dendritic cell migration and adaptive immune response by leukotriene B4 receptors: a role for LTB4 in up-regulation of CCR7 expression and function. Blood, 2007. 109(2): p. 626-31.

205. Nam, Y.H., et al., Leukotriene B(4) receptors BLT1 and BLT2 are involved in interleukin-8 production in human neutrophils induced by Trichomonas vaginalis-derived secretory products. Inflamm Res, 2012. 61(2): p. 97-102.

206. Woo, C.H., et al., Leukotriene B(4) stimulates Rac-ERK cascade to generate reactive oxygen species that mediates chemotaxis. J Biol Chem, 2002. 277(10): p. 8572-8.

207. Jozefowski, S., et al., Leukotrienes modulate cytokine release from dendritic cells. Immunology, 2005. 116(4): p. 418-28.

208. Brandt, S.L., et al., Macrophage-derived LTB4 promotes abscess formation and clearance of Staphylococcus aureus skin infection in mice. PLoS Pathog, 2018. 14(8): p. e1007244.

209. Tavares, N.M., et al., Understanding the mechanisms controlling Leishmania amazonensis infection in vitro: the role of LTB4 derived from human neutrophils. J Infect Dis, 2014. 210(4): p. 656-66.

210. Zhang, Y., R.M. Olson, and C.R. Brown, Macrophage LTB4 drives efficient phagocytosis of Borrelia burgdorferi via BLT1 or BLT2. J Lipid Res, 2017. 58(3): p. 494-503.

211. Berton, G., et al., Neutrophil activation by adhesion: mechanisms and pathophysiological implications. Int J Clin Lab Res, 1996. 26(3): p. 160-77.

212. Ekpenyong, A.E., et al., Mechanotransduction in neutrophil activation and deactivation. Biochim Biophys Acta, 2015. 1853(11 Pt B): p. 3105-16.

213. Ginis, I. and A.I. Tauber, Activation mechanisms of adherent human neutrophils. Blood, 1990. 76(6): p. 1233-9.

214. Sanui, T., et al., Adhesion attenuates respiratory burst induced by different modes of triggering in resting or LPS-primed neutrophils. Immunobiology, 2017. 222(8): p. 865871.

215. Arndt, P.G., et al., Lipopolysaccharide-induced c-Jun NH2-terminal kinase activation in human neutrophils: role of phosphatidylinositol 3-Kinase and Syk-mediated pathways. J Biol Chem, 2004. 279(12): p. 10883-91.

216. Futosi, K., S. Fodor, and A. Mócsai, Neutrophil cell surface receptors and their intracellular signal transduction pathways. International immunopharmacology, 2013. 17(3): p. 638-650.

217. Nick, J.A., et al., Common and distinct intracellular signaling pathways in human neutrophils utilized by platelet activating factor and FMLP. J Clin Invest, 1997. 99(5): p. 975-86. 
218. Zschaler, J., D. Schlorke, and J. Arnhold, Differences in Innate Immune Response between Man and Mouse. 2014. 34(5): p. 433-454.

219. Winther, M., C. Dahlgren, and H. Forsman, Formyl Peptide Receptors in Mice and Men: Similarities and Differences in Recognition of Conventional Ligands and Modulating Lipopeptides. Basic Clin Pharmacol Toxicol, 2018. 122(2): p. 191-198.

220. Chu, S.C., et al., Analysis of the Cytokine-Stimulated Human Inducible Nitric Oxide Synthase (iNOS) Gene: Characterization of Differences between Human and Mouse iNOS Promoters. Biochemical and Biophysical Research Communications, 1998. 248(3): p. 871-878.

221. Pechous, R.D., et al., Early host cell targets of Yersinia pestis during primary pneumonic plague. PLoS Pathog, 2013. 9(10): p. e1003679.

222. Stasulli, N.M., et al., Spatially Distinct Neutrophil Responses within the Inflammatory Lesions of Pneumonic Plague. MBio, 2015. 6(5).

223. Gater, S.T., et al., Host stress and immune responses during aerosol challenge of Brown Norway rats with Yersinia pestis. Front Cell Infect Microbiol, 2012. 2: p. 147.

224. Spinner, J.L., et al., Role of the Yersinia YopJ protein in suppressing interleukin-8 secretion by human polymorphonuclear leukocytes. Microbes Infect, 2016. 18(1): p. 219.

225. Lee, W.L., J.M. Grimes, and R.C. Robinson, Yersinia effector YopO uses actin as bait to phosphorylate proteins that regulate actin polymerization. Nat Struct Mol Biol, 2015. 22(3): p. 248-55.

226. Songsungthong, W., et al., ROS-inhibitory activity of YOPE is required for full virulence of Yersinia in mice. Cell Microbiol, 2010. 12(7): p. 988-1001.

227. Green, S.P., et al., Role of YopH in the suppression of tyrosine phosphorylation and respiratory burst activity in murine macrophages infected with Yersinia enterocolitica. J Leukoc Biol, 1995. 57(6): p. 972-7.

228. Pergola, C., et al., ERK-mediated regulation of leukotriene biosynthesis by androgens: a molecular basis for gender differences in inflammation and asthma. Proc Natl Acad Sci U S A, 2008. 105(50): p. 19881-6.

229. Palace, S.G., et al., Gain of Function Analysis Reveals Important Virulence Roles for the Yersinia pestis Type III Secretion System Effectors YopJ, YopT, and YpkA. Infect Immun, 2018.

230. Kinder, S.A., et al., Cloning of the Yenl restriction endonuclease and methyltransferase from Yersinia enterocolitica serotype 08 and construction of a transformable $R-M+$ mutant. Gene, 1993. 136(1-2): p. 271-5.

231. Haslett, C., et al., Modulation of multiple neutrophil functions by preparative methods or trace concentrations of bacterial lipopolysaccharide. Am J Pathol, 1985. 119(1): p. 10110.

232. SenGupta, S., et al., Human neutrophils depend on extrinsic factors produced by monocytes for their survival response to TLR4 stimulation. Innate immunity, 2019. 25(8): p. 473-486.

233. Taheri, N., A. Fahlgren, and M. Fallman, Yersinia pseudotuberculosis blocks neutrophil degranulation. Infect Immun, 2016.

234. Armstrong, C.L., et al., Filifactor alocis Promotes Neutrophil Degranulation and Chemotactic Activity. Infect Immun, 2016. 84(12): p. 3423-3433.

235. Mizuno-Yamasaki, E., F. Rivera-Molina, and P. Novick, GTPase networks in membrane traffic. Annu Rev Biochem, 2012. 81: p. 637-59. 
236. Lamber, E.P., A.C. Siedenburg, and F.A. Barr, Rab regulation by GEFs and GAPs during membrane traffic. Curr Opin Cell Biol, 2019. 59: p. 34-39.

237. Gavriljuk, K., et al., Membrane extraction of Rab proteins by GDP dissociation inhibitor characterized using attenuated total reflection infrared spectroscopy. Proc Natl Acad Sci U S A, 2013. 110(33): p. 13380-5.

238. Stenmark, H., Rab GTPases as coordinators of vesicle traffic. Nat Rev Mol Cell Biol, 2009. 10(8): p. 513-25.

239. Brumell, J.H. and M.A. Scidmore, Manipulation of rab GTPase function by intracellular bacterial pathogens. Microbiol Mol Biol Rev, 2007. 71(4): p. 636-52.

240. Chen, Y. and M.P. Machner, Targeting of the small GTPase Rab6A' by the Legionella pneumophila effector LidA. Infect Immun, 2013. 81(6): p. 2226-35.

241. Clemens, D.L., B.Y. Lee, and M.A. Horwitz, Deviant expression of Rab5 on phagosomes containing the intracellular pathogens Mycobacterium tuberculosis and Legionella pneumophila is associated with altered phagosomal fate. Infect Immun, 2000. 68(5): p. 2671-84.

242. Damiani, M.T., J. Gambarte Tudela, and A. Capmany, Targeting eukaryotic Rab proteins: a smart strategy for chlamydial survival and replication. Cell Microbiol, 2014. 16(9): p. 1329-38.

243. de Barsy, M., et al., Identification of a Brucella spp. secreted effector specifically interacting with human small GTPase Rab2. Cell Microbiol, 2011. 13(7): p. 1044-58.

244. Feng, Z.Z., et al., The Salmonella effectors SseF and SseG inhibit Rab1A-mediated autophagy to facilitate intracellular bacterial survival and replication. J Biol Chem, 2018. 293(25): p. 9662-9673.

245. Halaas, O., et al., Intracellular Mycobacterium avium intersect transferrin in the Rab11(+) recycling endocytic pathway and avoid lipocalin 2 trafficking to the lysosomal pathway. J Infect Dis, 2010. 201(5): p. 783-92.

246. Stein, M.-P., M.P. Müller, and A. Wandinger-Ness, Bacterial Pathogens Commandeer Rab GTPases to Establish Intracellular Niches. Traffic (Copenhagen, Denmark), 2012. 13(12): p. 1565-1588.

247. Thanassi, D.G. and S.J. Hultgren, Multiple pathways allow protein secretion across the bacterial outer membrane. Curr Opin Cell Biol, 2000. 12(4): p. 420-30.

248. Abdullah, Z. and P.A. Knolle, Scaling of immune responses against intracellular bacterial infection. The EMBO journal, 2014. 33(20): p. 2283-2294.

249. Thurston, T.L., et al., Galectin 8 targets damaged vesicles for autophagy to defend cells against bacterial invasion. Nature, 2012. 482(7385): p. 414-8.

250. Yu, H.B., et al., Autophagy facilitates Salmonella replication in HeLa cells. MBio, 2014. 5(2): p. e00865-14.

251. Escoll, P., M. Rolando, and C. Buchrieser, Modulation of Host Autophagy during Bacterial Infection: Sabotaging Host Munitions for Pathogen Nutrition. Front Immunol, 2016. 7: p. 81.

252. Sherwood, R.K. and C.R. Roy, A Rab-centric perspective of bacterial pathogen-occupied vacuoles. Cell Host Microbe, 2013. 14(3): p. 256-68.

253. Nokes, R.L., et al., Rab13 regulates membrane trafficking between TGN and recycling endosomes in polarized epithelial cells. J Cell Biol, 2008. 182(5): p. 845-53.

254. Magadan, J.G., et al., Rab22a regulates the sorting of transferrin to recycling endosomes. Mol Cell Biol, 2006. 26(7): p. 2595-614.

255. Evans, T.M., et al., Rab23, a negative regulator of hedgehog signaling, localizes to the plasma membrane and the endocytic pathway. Traffic, 2003. 4(12): p. 869-84. 
256. Egami, Y. and N. Araki, Rab20 regulates phagosome maturation in RAW264

macrophages during Fc gamma receptor-mediated phagocytosis. PLoS One, 2012. 7(4): p. e35663.

257. Jacob, A., et al., Rab40b regulates trafficking of MMP2 and MMP9 during invadopodia formation and invasion of breast cancer cells. J Cell Sci, 2013. 126(Pt 20): p. 4647-58.

258. Aizawa, M. and M. Fukuda, Small GTPase Rab2B and Its Specific Binding Protein Golgiassociated Rab2B Interactor-like 4 (GARI-L4) Regulate Golgi Morphology. J Biol Chem, 2015. 290(36): p. 22250-61.

259. Pei, G., et al., Identification of an immune-regulated phagosomal Rab cascade in macrophages. J Cell Sci, 2014. 127(Pt 9): p. 2071-82.

260. Seto, S., K. Tsujimura, and Y. Koide, Rab GTPases regulating phagosome maturation are differentially recruited to mycobacterial phagosomes. Traffic, 2011. 12(4): p. 407-20.

261. Schnettger, L., et al., A Rab20-Dependent Membrane Trafficking Pathway Controls M. tuberculosis Replication by Regulating Phagosome Spaciousness and Integrity. Cell Host Microbe, 2017. 21(5): p. 619-628.e5.

262. Weber, M.M. and R. Faris Subversion of the Endocytic and Secretory Pathways by Bacterial Effector Proteins. Frontiers in cell and developmental biology, 2018. 6, 1 DOI: 10.3389/fcell.2018.00001.

263. Ostrowski, M., et al., Rab27a and Rab27b control different steps of the exosome secretion pathway. Nat Cell Biol, 2010. 12(1): p. 19-30; sup pp 1-13.

264. de Bolle, X., J.J. Letesson, and J.P. Gorvel, Small GTPases and Brucella entry into the endoplasmic reticulum. Biochem Soc Trans, 2012. 40(6): p. 1348-52.

265. Guichard, A., V. Nizet, and E. Bier, RAB11-mediated trafficking in host-pathogen interactions. Nat Rev Microbiol, 2014. 12(9): p. 624-34.

266. Huang, B., et al., The Anaplasma phagocytophilum-occupied vacuole selectively recruits Rab-GTPases that are predominantly associated with recycling endosomes. Cell Microbiol, 2010. 12(9): p. 1292-307.

267. Ouellette, S.P. and R.A. Carabeo, A Functional Slow Recycling Pathway of Transferrin is Required for Growth of Chlamydia. Front Microbiol, 2010. 1: p. 112.

268. Mihai Gazdag, E., et al., Mechanism of Rab1b deactivation by the Legionella pneumophila GAP LepB. EMBO Rep, 2013. 14(2): p. 199-205.

269. Kauppi, M., et al., The small GTPase Rab22 interacts with EEA1 and controls endosomal membrane trafficking. J Cell Sci, 2002. 115(Pt 5): p. 899-911.

270. Shakya, S., et al., Rab22A recruits BLOC-1 and BLOC-2 to promote the biogenesis of recycling endosomes. EMBO Rep, 2018. 19(12).

271. Weigert, R., et al., Rab22a regulates the recycling of membrane proteins internalized independently of clathrin. Mol Biol Cell, 2004. 15(8): p. 3758-70.

272. Carlyon, J.A., et al., Anaplasma phagocytophilum utilizes multiple host evasion mechanisms to thwart NADPH oxidase-mediated killing during neutrophil infection. Infect Immun, 2004. 72(8): p. 4772-83.

273. Price, P.A., J. Jin, and W.E. Goldman, Pulmonary infection by Yersinia pestis rapidly establishes a permissive environment for microbial proliferation. Proc Natl Acad Sci U S A, 2012. 109(8): p. 3083-8.

274. Bubeck, S.S., A.M. Cantwell, and P.H. Dube, Delayed inflammatory response to primary pneumonic plague occurs in both outbred and inbred mice. Infect Immun, 2007. 75(2): p. 697-705.

275. Comer, J.E., E.A. Lorange, and B.J. Hinnebusch, Examining the vector-host-pathogen interface with quantitative molecular tools. Methods Mol Biol, 2008. 431: p. 123-31. 
276. Mocsai, A., B. Walzog, and C.A. Lowell, Intracellular signalling during neutrophil recruitment. Cardiovasc Res, 2015. 107(3): p. 373-85.

277. Kobayashi, S.D., N. Malachowa, and F.R. DeLeo, Neutrophils and Bacterial Immune Evasion. J Innate Immun, 2018. 10(5-6): p. 432-441.

278. Yin, C. and B. Heit, Armed for destruction: formation, function and trafficking of neutrophil granules. Cell Tissue Res, 2018. 371(3): p. 455-471.

279. Scapini, P. and M.A. Cassatella, Social networking of human neutrophils within the immune system. Blood, 2014. 124(5): p. 710-9.

280. Tecchio, C. and M.A. Cassatella, Neutrophil-derived chemokines on the road to immunity. Semin Immunol, 2016. 28(2): p. 119-28.

281. el-Ahmady, O., et al., Elevated concentrations of interleukins and leukotriene in response to Mycobacterium tuberculosis infection. Ann Clin Biochem, 1997. 34 ( Pt 2): p. 160-4.

282. Min, A., et al., BLT1-mediated O-GlcNAcylation is required for NOX2-dependent migration, exocytotic degranulation and IL-8 release of human mast cell induced by Trichomonas vaginalis-secreted LTB4. Microbes Infect, 2018. 20(6): p. 376-384.

283. Spinner, J.L., et al., Role of Yersinia pestis toxin complex family proteins in resistance to phagocytosis by polymorphonuclear leukocytes. Infect Immun, 2013. 81(11): p. 4041-52.

284. Spinner, J.L., et al., Neutrophils are resistant to Yersinia YopJ/P-induced apoptosis and are protected from ROS-mediated cell death by the type III secretion system. PLoS One, 2010. 5(2): p. e9279.

285. Rolan, H.G., E.A. Durand, and J. Mecsas, Identifying Yersinia YopH-targeted signal transduction pathways that impair neutrophil responses during in vivo murine infection. Cell Host Microbe, 2013. 14(3): p. 306-17.

286. Wiley, D.J., et al., The Ser/Thr kinase activity of the Yersinia protein kinase A (YpkA) is necessary for full virulence in the mouse, mollifying phagocytes, and disrupting the eukaryotic cytoskeleton. Microb Pathog, 2006. 40(5): p. 234-43.

287. Groves, E., et al., Sequestering of Rac by the Yersinia effector YopO blocks Fcgamma receptor-mediated phagocytosis. J Biol Chem, 2010. 285(6): p. 4087-98.

288. Singaravelu, P., et al., Yersinia effector protein (YopO)-mediated phosphorylation of host gelsolin causes calcium-independent activation leading to disruption of actin dynamics. J Biol Chem, 2017. 292(19): p. 8092-8100.

289. Viboud, G.I., E. Mejia, and J.B. Bliska, Comparison of YopE and YopT activities in counteracting host signalling responses to Yersinia pseudotuberculosis infection. Cell Microbiol, 2006. 8(9): p. 1504-15.

290. Dave, M.N., et al., Yersinia enterocolitica YopH-deficient Strain Activates Neutrophil Recruitment to Peyer's patches Promoting Clearance of the Virulent Strain. Infect Immun, 2016.

291. Montagna, L.G., M.I. Ivanov, and J.B. Bliska, Identification of residues in the N-terminal domain of the Yersinia tyrosine phosphatase that are critical for substrate recognition. J Biol Chem, 2001. 276(7): p. 5005-11.

292. Dukuzumuremyi, J.M., et al., The Yersinia protein kinase $A$ is a host factor inducible RhoA/Rac-binding virulence factor. J Biol Chem, 2000. 275(45): p. 35281-90.

293. Ke, Y., et al., Yersinia protein kinase A phosphorylates vasodilator-stimulated phosphoprotein to modify the host cytoskeleton. Cell Microbiol, 2015. 17(4): p. 473-85.

294. Navarro, L., et al., Identification of a molecular target for the Yersinia protein kinase A. Mol Cell, 2007. 26(4): p. 465-77. 
295. Wiley, D.J., et al., The activities of the Yersinia protein kinase $A(Y p k A)$ and outer protein $J(Y o p J)$ virulence factors converge on an elF2alpha kinase. J Biol Chem, 2009. 284(37): p. 24744-53.

296. Mecsas, J., Unraveling neutrophil- Yersinia interactions during tissue infection. F1000Res, 2019. 8.

297. Zheng, Y., et al., A Yersinia effector with enhanced inhibitory activity on the NF-kappaB pathway activates the NLRP3/ASC/caspase-1 inflammasome in macrophages. PLoS Pathog, 2011. 7(4): p. e1002026.

298. Casson, C.N., et al., Caspase-11 activation in response to bacterial secretion systems that access the host cytosol. PLoS Pathog, 2013. 9(6): p. e1003400.

299. Philip, N.H., et al., Caspase-8 mediates caspase-1 processing and innate immune defense in response to bacterial blockade of NF-kappaB and MAPK signaling. Proc Natl Acad Sci U S A, 2014. 111(20): p. 7385-90.

300. Philip, N.H., E.E. Zwack, and I.E. Brodsky, Activation and Evasion of Inflammasomes by Yersinia. Curr Top Microbiol Immunol, 2016. 397: p. 69-90.

301. Rosadini, C.V., et al., A Single Bacterial Immune Evasion Strategy Dismantles Both MyD88 and TRIF Signaling Pathways Downstream of TLR4. Cell Host Microbe, 2015. 18(6): p. 682-93.

302. Orning, P., et al., Pathogen blockade of TAK1 triggers caspase-8-dependent cleavage of gasdermin D and cell death. Science, 2018.

303. Viboud, G.I. and J.B. Bliska, Yersinia outer proteins: role in modulation of host cell signaling responses and pathogenesis. Annu Rev Microbiol, 2005. 59: p. 69-89.

304. Wei, C., et al., The Yersinia Type III secretion effector YopM Is an E3 ubiquitin ligase that induced necrotic cell death by targeting NLRP3. Cell Death Dis, 2016. 7(12): p. e2519.

305. Cheng, L.W., D.M. Anderson, and O. Schneewind, Two independent type III secretion mechanisms for YopE in Yersinia enterocolitica. Mol Microbiol, 1997. 24(4): p. 757-65.

306. Fahlgren, A., et al., Cell type-specific effects of Yersinia pseudotuberculosis virulence effectors. Cell Microbiol, 2009. 11(12): p. 1750-67.

307. Orth, K., et al., Inhibition of the mitogen-activated protein kinase kinase superfamily by a Yersinia effector. Science, 1999. 285(5435): p. 1920-3.

308. Sweet, C.R., et al., YopJ targets TRAF proteins to inhibit TLR-mediated NF-kappaB, MAPK and IRF3 signal transduction. Cell Microbiol, 2007. 9(11): p. 2700-15.

309. Meijer, L.K., et al., The bacterial protein YopJ abrogates multiple signal transduction pathways that converge on the transcription factor CREB. Cell Microbiol, 2000. 2(3): p. 231-8.

310. Orth, K., et al., Disruption of signaling by Yersinia effector YopJ, a ubiquitin-like protein protease. Science, 2000. 290(5496): p. 1594-7.

311. Palmer, L.E., et al., YopJ of Yersinia pseudotuberculosis is required for the inhibition of macrophage TNF-alpha production and downregulation of the MAP kinases $p 38$ and JNK. Mol Microbiol, 1998. 27(5): p. 953-65.

312. Paquette, N., et al., Serine/threonine acetylation of TGFbeta-activated kinase (TAK1) by Yersinia pestis YopJ inhibits innate immune signaling. Proc Natl Acad Sci U S A, 2012. 109(31): p. 12710-5.

313. Sylvain-Prevost, S., et al., Activation of TAK1 by Chemotactic and Growth Factors, and Its Impact on Human Neutrophil Signaling and Functional Responses. J Immunol, 2015. 195(11): p. 5393-403. 
314. Mittal, R., S.Y. Peak-Chew, and H.T. McMahon, Acetylation of MEK2 and I kappa B kinase (IKK) activation loop residues by YopJ inhibits signaling. Proc Natl Acad Sci U S A, 2006. 103(49): p. 18574-9.

315. Anderson, D.P., Adjuvants and immunostimulants for enhancing vaccine potency in fish. Dev Biol Stand, 1997. 90: p. 257-65.

316. Wakabayashi, Y., et al., Effects of surface modification of materials on human neutrophil activation. Biochimica et Biophysica Acta (BBA) - General Subjects, 1995. 1243(3): p. 521-528.

317. Kinkead, L.C. and L.A. Allen, Multifaceted effects of Francisella tularensis on human neutrophil function and lifespan. Immunol Rev, 2016. 273(1): p. 266-81.

318. Eichelberger, K.R., G.S. Jones, and W.E. Goldman, Inhibition of Neutrophil Primary Granule Release during Yersinia pestis Pulmonary Infection. MBio, 2019. 10(6).

319. Lee, W.L., et al., Mechanisms of Yersinia YopO kinase substrate specificity. Sci Rep, 2017. 7: p. 39998.

320. Ghosh, S. and T.J. O'Connor, Beyond Paralogs: The Multiple Layers of Redundancy in Bacterial Pathogenesis. Front Cell Infect Microbiol, 2017. 7: p. 467.

321. Immler, R., S.I. Simon, and M. Sperandio, Calcium signalling and related ion channels in neutrophil recruitment and function. Eur J Clin Invest, 2018. 48 Suppl 2: p. e12964.

322. Hegde, B., et al., Inflammasome-Independent Leukotriene B4 Production Drives Crystalline Silica-Induced Sterile Inflammation. J Immunol, 2018. 200(10): p. 3556-3567.

323. Pacheco, P., et al., Lipopolysaccharide-induced leukocyte lipid body formation in vivo: innate immunity elicited intracellular Loci involved in eicosanoid metabolism. J Immunol, 2002. 169(11): p. 6498-506.

324. Lepley, R.A. and F.A. Fitzpatrick, 5-Lipoxygenase contains a functional Src homology 3binding motif that interacts with the Src homology 3 domain of Grb2 and cytoskeletal proteins. J Biol Chem, 1994. 269(39): p. 24163-8.

325. Forsberg, M., et al., Tumour necrosis factor-alpha potentiates CR3-induced respiratory burst by activating p38 MAP kinase in human neutrophils. Immunology, 2001. 103(4): p. 465-472.

326. Zhou, J., et al., BLT1 in dendritic cells promotes Th1/Th17 differentiation and its deficiency ameliorates TNBS-induced colitis. Cellular \& Molecular Immunology, 2018. 15(12): p. 1047-1056.

327. Toda, A., et al., Attenuated Th1 induction by dendritic cells from mice deficient in the leukotriene B4 receptor 1. Biochimie, 2010. 92(6): p. 682-91.

328. Gavriljuk, K., et al., Reaction mechanism of adenylyltransferase DrrA from Legionella pneumophila elucidated by time-resolved fourier transform infrared spectroscopy. J Am Chem Soc, 2014. 136(26): p. 9338-45.

329. Hardiman, C.A. and C.R. Roy, AMPylation is critical for Rab1 localization to vacuoles containing Legionella pneumophila. mBio, 2014. 5(1): p. e01035-13.

330. Mukherjee, S., et al., Modulation of Rab GTPase function by a protein phosphocholine transferase. Nature, 2011. 477(7362): p. 103-6.

331. Muller, M.P., et al., The Legionella effector protein DrrA AMPylates the membrane traffic regulator Rab1b. Science, 2010. 329(5994): p. 946-9.

332. Muller, M.P., et al., Characterization of enzymes from Legionella pneumophila involved in reversible adenylylation of Rab1 protein. J Biol Chem, 2012. 287(42): p. 35036-46.

333. Tan, Y. and Z.Q. Luo, Legionella pneumophila SidD is a deAMPylase that modifies Rab1. Nature, 2011. 475(7357): p. 506-9. 
334. Mallo, G.V., et al., SopB promotes phosphatidylinositol 3-phosphate formation on Salmonella vacuoles by recruiting Rab5 and Vps34. J Cell Biol, 2008. 182(4): p. 741-52.

335. Chen, A.L., et al., Novel components of the Toxoplasma inner membrane complex revealed by BiolD. MBio, 2015. 6(1): p. e02357-14.

336. O'Loughlin, T., T.A. Masters, and F. Buss, The MYO6 interactome reveals adaptor complexes coordinating early endosome and cytoskeletal dynamics. EMBO Rep, 2018.

337. Molleken, K. and J.H. Hegemann, Acquisition of Rab11 and Rab11-Fip2-A novel strategy for Chlamydia pneumoniae early survival. PLoS Pathog, 2017. 13(8): p. e1006556.

338. Osei-Owusu, P., et al., FPR1 is the plague receptor on host immune cells. Nature, 2019.

339. Zhang, S.S., et al., Plasminogen activator Pla of Yersinia pestis utilizes murine DEC-205 (CD205) as a receptor to promote dissemination. J Biol Chem, 2008. 283(46): p. 3151121.

340. Zhang, P., et al., Human dendritic cell-specific intercellular adhesion molecule-grabbing nonintegrin (CD209) is a receptor for Yersinia pestis that promotes phagocytosis by dendritic cells. Infect Immun, 2008. 76(5): p. 2070-9.

341. Tan, Y. and J.C. Kagan, Microbe-inducible trafficking pathways that control Toll-like receptor signaling. Traffic (Copenhagen, Denmark), 2017. 18(1): p. 6-17.

342. Klein, D.C., et al., CD14, TLR4 and TRAM Show Different Trafficking Dynamics During LPS Stimulation. Traffic, 2015. 16(7): p. 677-90.

343. Skjesol, A., et al., The TLR4 adaptor TRAM controls the phagocytosis of Gram-negative bacteria by interacting with the Rab11-family interacting protein 2. PLoS Pathog, 2019. 15(3): p. e1007684.

344. Neal, M.D., et al., Enterocyte TLR4 mediates phagocytosis and translocation of bacteria across the intestinal barrier. J Immunol, 2006. 176(5): p. 3070-9.

345. Werz, O., 5-lipoxygenase: cellular biology and molecular pharmacology. Curr Drug Targets Inflamm Allergy, 2002. 1(1): p. 23-44.

346. Gerstmeier, J., et al., Time-resolved in situ assembly of the leukotriene-synthetic 5lipoxygenase/5-lipoxygenase-activating protein complex in blood leukocytes. Faseb j, 2016. 30(1): p. 276-85.

347. Yaffe, M.B., et al., Priming of the neutrophil respiratory burst is species-dependent and involves MAP kinase activation. Surgery, 1999. 126(2): p. 248-254.

348. Suzuki, K., et al., Cytokine-specific activation of distinct mitogen-activated protein kinase subtype cascades in human neutrophils stimulated by granulocyte colony-stimulating factor, granulocyte-macrophage colony-stimulating factor, and tumor necrosis factoralpha. Blood, 1999. 93(1): p. 341-9.

349. Partrick, D.A., et al., Maximal Human Neutrophil Priming for Superoxide Production and Elastase Release Requires p38 Mitogen-Activated Protein Kinase Activation. Archives of Surgery, 2000. 135(2): p. 219-225.

350. Tardif, M.R., et al., Secretion of S100A8, S100A9, and S100A12 by Neutrophils Involves Reactive Oxygen Species and Potassium Efflux. J Immunol Res, 2015. 2015: p. 296149.

351. Jean, S., et al., Neisseria gonorrhoeae Evades Calprotectin-Mediated Nutritional Immunity and Survives Neutrophil Extracellular Traps by Production of TdfH. Infection and immunity, 2016. 84(10): p. 2982-2994.

352. Casutt-Meyer, S., et al., Oligomeric coiled-coil adhesin YadA is a double-edged sword. PLoS One, 2010. 5(12): p. e15159.

353. Meldolesi, J., Exosomes and Ectosomes in Intercellular Communication. Curr Biol, 2018. 28(8): p. R435-r444. 
354. Villarroya-Beltri, C., et al., Sorting it out: regulation of exosome loading. Seminars in cancer biology, 2014. 28: p. 3-13.

355. Zhang, W., et al., Exosomes in Pathogen Infections: A Bridge to Deliver Molecules and Link Functions. Frontiers in immunology, 2018. 9: p. 90-90.

356. Hoshino, D., et al., Exosome secretion is enhanced by invadopodia and drives invasive behavior. Cell reports, 2013. 5(5): p. 1159-1168.

357. Kowal, J., M. Tkach, and C. Thery, Biogenesis and secretion of exosomes. Curr Opin Cell Biol, 2014. 29: p. 116-25.

358. Trasak, C., et al., Yersinia protein kinase YopO is activated by a novel G-actin binding process. J Biol Chem, 2007. 282(4): p. 2268-77.

359. Singh, P.P., et al., Exosomes isolated from mycobacteria-infected mice or cultured macrophages can recruit and activate immune cells in vitro and in vivo. J Immunol, 2012. 189(2): p. 777-85.

360. Konig, S., et al., Gliotoxin from Aspergillus fumigatus Abrogates Leukotriene B4 Formation through Inhibition of Leukotriene A4 Hydrolase. Cell Chem Biol, 2019. 26(4): p. 524-534.e5.

361. Eichelberger, K.R., G.S. Jones, and W.E. Goldman, Inhibition of Neutrophil Primary Granule Release during Yersinia pestis Pulmonary Infection. mBio, 2019. 10(6): p. e02759-19. 


\section{APPENDIX}

Chapter III has previously been published, the acknowledgements are listed below. Chapter III was published in Infection and Immunity, which is an ASM owned journal. The journal gives permission to publish Chapter III under the exemption of dissertations.

\section{Chapter IV}

\section{Acknowledgements}

We would like to thank Terri Manning, Irina Miralda, and Keith C. Klaes for isolation of human neutrophils and for helpful discussions. This work was supported by NIH grants GM125504 and Al119557 (MBL), a Collaborative Research Grant from the University of Louisville's Center for Predictive Medicine for Biodefense and Emerging Infectious Disease (MBL and SMU), and in part by a grant from the Jewish Heritage Fund for Excellence Research Enhancement Grant Program at the University of Louisville, School of Medicine (MBL). 


\title{
CURRICULUM VITAE
}

\author{
Amanda Rose Pulsifer \\ Dept. of Microbiology and Immunology \\ 505 S Hancock Street Rm 633D \\ Louisville, KY 40202 \\ Email: arpuls01@louisville.edu
}

EDUCATION

Bloomsburg University of Pennsylvania (GPA: 3.95)

August 2008 - May 2012

Bloomsburg, Pennsylvania

B. S. Biology

Minor: Chemistry

Graduated: Summa Cum Laude

University of Louisville (GPA: 3.90)

Louisville, Kentucky

M.S. Microbiology and Immunology

August 2013 - Present

Mentor: Matthew Lawrenz, Ph.D.

June 2015

Ph.D. Microbiology and Immunology

March 2020

Graduate Dean's Citation Recipient

Mentor: Matthew Lawrenz, Ph.D

Dissertation title: Modulation of host innate immune cells by Yersinia pestis to create a permissive environment for replication

HONORS AND AWARDS

- Geisinger Janet Weis- Research Internship

May - August 2011

Danville, Pennsylvania

- Phi Kappa Phi (Interdisciplinary Honors Society)

April $10^{\text {th }} 2011$

- Avanti Poster Prize -top poster presentation at Phagocytes Gordon Research

Conference.

The Yin and Yang of Phagocytes: Regulators of Human Health and Disease.

Waterville Valley, $\mathrm{NH}$

June 15, 2017

- Midwest Microbial Pathogenesis Conference Travel Award for encouraging young scientists to present research at a conference. (Awarded based on Abstract) Notre Dame- North Bend, IN

August 25-27, 2017

- Presidential Award Finalist and Travel Award Recipient-One of three top abstracts selected to present at Society of Leukocyte Biology Conference. Chandler, AZ

October 15, 2018

\section{ORAL PRESENTATIONS}

Regional and International Meetings

1. COST Research Day, (Dec $\left.9^{\text {th }}, 2011\right)$. Bloomsburg University of Pennsylvania, Bloomsburg, PA

"The Role of YAP in Hydra Regeneration"

2. Microbiology \& Immunology Seminar Series (Dec 17 $\left.7^{\text {th }}, 2015\right)$. University of Louisville, Louisville, KY. "Exploitation of host Rab proteins by Yersinia pestis during intracellular infection"

3. Microbiology \& Immunology Seminar Series (February $\left.9^{\text {th }}, 2017\right)$. University of Louisville, Louisville, KY. "Interplay of Yersinia pestis with neutrophils and macrophages." 
4. Center for Predictive Medicine Retreat (November $\left.15^{\text {th }}, 2016\right)$. University of Louisville, Louisville, KY. "Response of human neutrophils to Yersinia pestis infection."

5. ASM Biothreats Conference (February 11, 2018). Baltimore, MD. "Exploitation of Host Rab GTPases by Yersinia pestis Facilitates Intracellular Survival"

6. Society of Leukocyte Biology (October 15, 2018). "Mico manipulator: Yersinia pestis actively alters the neutrophil response". Chandler, AZ.

7. Center for Predictive Medicine Research Symposium (February 25, 2019). "Yersinia pestits inhibits PMN degranulation." University of Louisville, Hurstbourne, KY.

\section{POSTER PRESENTATIONS}

Regional and International Meetings

1. Research! Louisville, Louisville, KY (Sept 16 ${ }^{\text {th }}$, 2014). "Host Rab1b Is Required for Yersinia pestis Intracellular Survival"; *Amanda R. Pulsifer, Michael G. Connor, Jarrod M. Pennington, and Matthew B Lawrenz.

2. Membrane Trafficking and Signaling Symposium, Louisville, KY (October $3^{\text {rd }}, 2014$ ). "Host Rab1b Is Required for Yersinia pestis Intracellular Survival"; *Amanda R. Pulsifer, Michael G. Connor, Jarrod M. Pennington, and Matthew B. Lawrenz.

3. Research! Louisville, Louisville, KY (2015). "Manipulation of Host Rab GTPases by $Y$. pestis"; *Amanda R. Pulsifer, Michael G. Connor, and Matthew B Lawrenz.

4. Midwest Microbial Pathogenesis Conference. Urbana Champaigne, IL (Sept 24 ${ }^{\text {th }}, 2016$ ). "Exploitation of Host Rab GTPase by Y. pestis"; *Amanda R. Pulsifer, Michael G. Connor, and Matthew B Lawrenz.

5. Gordon Research Conference, The Yin and Yang of Phagocytes, Waterville Valley, NH (June 2017). "Exploitation of Host Rab GTPases by Yersinia pestis Facilitates Intracellular Survival"; *Amanda R. Pulsifer, Michael G. Connor, Donghoon Chung, Eric C. Rouchka, and Matthew B. Lawrenz.

6. Midwest Microbial Pathogenesis Conference. Notre Dame, IN (Sept $9^{\text {th }}, 2016$ ). "Exploitation of Host Rab GTPases by Yersinia pestis Facilitates Intracellular Survival"; *Amanda R. Pulsifer, Michael G. Connor, Donghoon Chung, Eric C. Rouchka, and Matthew B. Lawrenz.

7. ASM Biothreats Conference. Baltimore, MD (Feb 13 $\left.{ }^{\text {th }}, 2018\right)$. "Exploitation of Host Rab GTPases by Yersinia pestis Facilitates Intracellular Survival"; *Amanda R. Pulsifer, Michael G. Connor, Donghoon Chung, Eric C. Rouchka, and Matthew B. Lawrenz.

8. Research! Louisville, Louisville, KY (2018). "Yerisina pestis actively inhibits PMN degranulation"; *Amanda R. Pulsifer, Shane A. Reeves, Sobha Bodduluri, Haribabu Bodduluri, Silvia M. Uriarte, and Matthew B Lawrenz.

9. Society of Leukocyte Biology Conference. Chandler, AZ (Oct $\left.9^{\text {th }}, 2018\right)$. "Mico manipulator: Yersinia pestis actively alters the neutrophil response"; *Amanda R. Pulsifer, Shane A. Reeves, Sobha Bodduluri, Haribabu Bodduluri, Silvia M. Uriarte, and Matthew B. Lawrenz.

\section{PUBLICATIONS}

1. Connor, M.G., A.R. Pulsifer, C.T. Price, Y. Abu Kwaik, M.B. Lawrenz. 2015. Yersinia pestis requires host Rab1b for survival in macrophages.PLoS Pathog. 2015 Oct 23;11(10):e1005241. eCollection 2015 Oct.

2. VanCleave, T.T., A. R. Pulsifer, M.G. Connor, J.M. Warawa and M. B. Lawrenz. 2017. Impact of gentamicin concentration and exposure time on intracellular Yersinia pestis, Frontiers in Cellular and Infection Microbiology

3. Connor, M.G., A.R. Pulsifer, D.H. Chung, E. Rouchka, B. K. Ceresa, M.B. Lawrenz. 2018. Yersinia pestis targets the host endosome recycling pathway during the biogenesis of the Yersinia containing vacuole to avoid killing by macrophages. mBio. 2018 Jan 23;11(10):e1005241. eCollection 2018 Jan.

4. Pulsifer A.R., VanCleave T.T., Lawrenz M.B. (2019) Intracellular Assays to Monitor Survival and Growth of Yersinia pestis Within Macrophages. In: Vadyvaloo V., Lawrenz M. (eds) Pathogenic Yersinia. Methods in Molecular Biology, vol 2010. Humana, New York, NY. 
5. Bowen W., L. Batra, A.R. Pulsifer, E.S. Yolcu, M.B. Lawrenz, and H. Shirwan. 2019. Timing of administration of SA-4-1BBL adjuvant influences protective efficacy of $\mathrm{rF} 1-\mathrm{V}$ vaccine against plague. Vaccine. 10;37(38):5708-5716. eCollection 2019.

6. Pulsifer, A.R., Vashishta A., Reeves, S.A., Wolfe, J.K., Palace S.G., Proulx M.K., Goguen J., Bodduluri, S.R., Haribabu B., Uriarte, S.M., Lawrenz, M.B. 2020. Redundant and cooperative roles for Yersinia pestis Yop effectors in the inhibition of human neutrophil exocytic responses revealed by gain-of-function approach. Infect Immun 88:e00909-19. eCollection 2020.

\section{MENTORSHIP}

Individuals trained in laboratory techniques:

- Tiva VanCleave - Confocal Microscopy (General and Live cell), Mammalian cell culture, in vitro infections, CFU enumeration

- Sarah Price - Animal models of infection (Intranasal inoculation, intradermal needle inoculation), animal husbandry

- Stephanie Lunn - Sterile Technique, Mammalian cell culture, in vitro infections, CFU enumeration

- Shane Reeves - Sterile Technique, PCR/Cloning

- Amanda Brady - Sterile Technique, mammalian cell culture, animal husbandry, RNA interference, mammalian cell plasmid based protein overexpression, bacterial electroporation, PCR/Cloning, CFU enumeration, confocal microscopy.

\section{COMMUNITY ENGAGEMENT}

Member of:

1. Tri Beta (Biology Honors Society)

2. Science Policy and Outreach Group

Fall 2011-Spring 2012

- Treasurer

Sept 2014 - Sept 2015

3. Microbiology and Immunology Student Organization

- Treasurer

- President

August 2014-July 2015

August 2016-July 2017

Volunteer activities:

- Assisted with set-up of Annual Health Sciences Symposium for nursing and allied health students (April 14 $4^{\text {th }}, 2011$ )

- Volunteered at the Tri Beta Northeast District Conference. (March 2012)

- Siblings and Children's weekend science demonstrations with Tri-beta club. (May 2012)

- Assisted with activities and lead a demonstration for high school students on microbial testing to introduce graduate study as a career option with SPOG outreach (August $15^{\text {th }}$, 2015)

- Volunteered for departmental recruitment tours, poster sessions, and speaking to recruits about life in Grad school and Louisville in general. (2014-2019)

- Welcomed incoming $1^{\text {st }}$ year IPIBS students and talked about life as a graduate student, what to expect for their Ph.D. and pitfalls to avoid. (August $4^{\text {th }}, 2017$ )

- Girls Rule STEM-H Summit- interacted with attending parents by escorting them around campus discussing the STEM-H related research and activities that University of Louisville hosts. (April $6^{\text {th }}, 2019$ )

- MISTRE committee member to select high school students from underprivileged areas to complete a summer internship program with the Department of Microbiology and Immunology at the University of Louisville. (May 2019) 